KUNS-2467

\title{
A deformation of quantum affine algebra in squashed WZNW models
}

\author{
Io Kawaguchi* and Kentaroh Yoshidat \\ Department of Physics, Kyoto University \\ Kyoto 606-8502, Japan
}

\begin{abstract}
We proceed to study infinite-dimensional symmetries in two-dimensional squashed Wess-Zumino-Novikov-Witten (WZNW) models at the classical level. The target space is given by squashed $S^{3}$ and the isometry is $S U(2)_{\mathrm{L}} \times U(1)_{\mathrm{R}}$. It is known that $S U(2)_{\mathrm{L}}$ is enhanced to a couple of Yangians. We reveal here that an infinite-dimensional extension of $U(1)_{\mathrm{R}}$ is a deformation of quantum affine algebra, where a new deformation parameter is provided with the coefficient of the Wess-Zumino term. Then we consider the relation between the deformed quantum affine algebra and the pair of Yangians from the viewpoint of the left-right duality of monodromy matrices. The integrable structure is also discussed by computing the $r / s$-matrices that satisfy the extended classical Yang-Baxter equation. Finally two degenerate limits are discussed.
\end{abstract}

${ }^{*}$ E-mail: io_at_gauge.scphys.kyoto-u.ac.jp

${ }^{\dagger}$ E-mail: kyoshida_at_gauge.scphys.kyoto-u.ac.jp 


\section{Contents}

1 Introduction 1

2 Preliminary

2.1 Squashed $S^{3} \ldots \ldots \ldots \ldots \ldots$

2.2 The classical action of the squashed WZNW models . . . . . . . . . . 4

3 The left description $\quad 6$

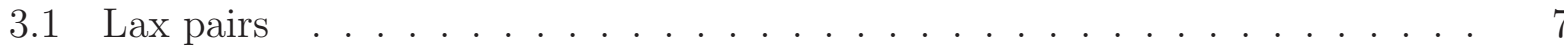

3.2 Yangians . . . . . . . . . . . . . . . . . . . . . . . . . . . 9

4 The right description $\quad 11$

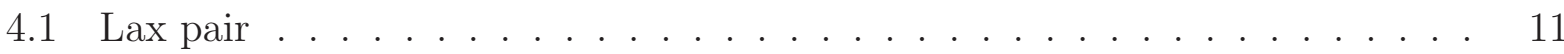

4.2 q-deformation of $s u(2)_{\mathrm{R}} \ldots \ldots \ldots \ldots \ldots$

4.3 Expansions of the monodromy matrix . . . . . . . . . . . . 15

4.4 An infinite-dimensional extension of $q$-deformed $s u(2)_{\mathrm{R}} \ldots \ldots . . . . . . \quad 19$

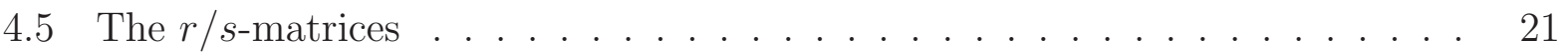

5 The left-right duality $\quad 23$

6 The degenerate limits

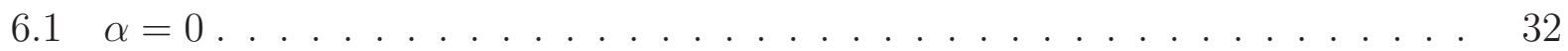

$6.2 \alpha=\pi i / 2 \ldots \ldots \ldots \ldots \ldots \ldots \ldots$

7 Conclusion and Discussion

A The Poisson brackets of $J_{\mu}^{a}$ and $j_{\mu}^{L_{ \pm}}$

A.1 The Poisson brackets of $J_{\mu}^{a} \ldots \ldots \ldots \ldots$. . . . . . . . . . . . . . . . . . . .

A.2 The current algebra of $j_{\mu}^{L_{ \pm}} \ldots \ldots \ldots \ldots \ldots$. . . . . . . . . . . . . . .

B A prescription to treat non-ultra local terms

B.1 Yangian $\mathcal{Y}\left(s u(2)_{\mathrm{L}}\right) \ldots \ldots \ldots \ldots \ldots \ldots \ldots$

B.2 q-deformed $s u(2)_{\mathrm{R}} \ldots \ldots \ldots \ldots \ldots \ldots \ldots$

B.3 A deformation of quantum affine algebra . . . . . . . . . . . 52 


\section{Introduction}

The AdS/CFT correspondence [13] has been a fascinating topic in the study of string theory over the decade after Maldacena's proposal. A tremendous amount of works have been devoted to test and generalize it. Nowadays, the research area covers diverse subjects. A great achievement in the recent progress is the discovery of the integrable structure behind the AdS/CFT correspondence (For a comprehensive review on this subject, see [4]).

In the string-theory side, the integrable structure of two-dimensional string sigma-models with target spacetime $\mathrm{AdS}_{5} \times \mathrm{S}^{5}$ plays an important role [5]. It is closely related to the fact that $\mathrm{AdS}_{5} \times \mathrm{S}^{5}$ is described as a symmetric coset. It leads to an infinite number of the conserved charges constructed, for example, by following the pioneering works [6 10] (For a comprehensive textbook see [11]). The symmetric cosets that potentially may lead to a holographic interpretation are classified, including spacetime fermions [12].

The next interesting issue is to consider integrable deformations of the AdS/CFT correspondence. There are two approaches. The one is an algebraic approach based on $q^{-}$ deformations of the world-sheet S-matrix [13 17]. The deformed S-matrices are explicitly constructed, while the target-space geometry is unclear. The other is a geometric approach based on deformations of target spaces of the sigma models. It seems likely that the deformed geometries are represented by non-symmetric cosets [18] in comparison to $\operatorname{AdS}_{5} \times \mathrm{S}^{5}$ and hence the prescription for symmetric cosets is not available any more. It is necessary to develop a new method to argue the integrability.

In the latter approach, there is a long history (For classic papers see [19 21]). Motivated by integrable deformations of AdS/CFT, a series of works have been done on squashed $\mathrm{S}^{3}$ and warped $\mathrm{AdS}_{3}[22 \sqrt{30}$. Some specific higher-dimensional cases are discussed in [31]. Remarkably, the classical integrable structure of deformed sigma models was recently shown for arbitrary compact Lie groups and the coset cousins by Delduc, Magro and Vicedo [32]. Then, they successively presented a $q$-deformation of the $\mathrm{AdS}_{5} \times \mathrm{S}^{5}$ superstring [33].

Based on the latter approach, we are here concerned with the classical integrable structure of two-dimensional Wess-Zumino-Novikov-Witten (WZNW) models with the target space squashed $\mathrm{S}^{3}$. The isometry is given by $S U(2)_{\mathrm{L}} \times U(1)_{\mathrm{R}}$. It is partly explained in [24] that there exist a couple of Yangian algebras based on $S U(2)_{\mathrm{L}}$ by explicit constructions of non-local conserved charges and direct computations of the Poisson brackets of the charges. 
In this paper we consider an infinite-dimensional extension of $U(1)_{\mathrm{R}}$. In the case without the Wess-Zumino term, it is just a classical analogue of a quantum affine algebra [26]. When the Wess-Zumino term is added, an additional constant parameter is introduced as its coefficient. A natural question is what happens to the quantum affine algebra. As one may easily guess, a new kind of deformation is induced by the presence of the Wess-Zumino term. The resulting algebra is a classical analogue of the deformed quantum affine algebra. So far, it is not clear what is the mathematical formulation of the deformed quantum affine algebra, though it seems likely to be a two-parameter quantum toroidal algebra [34]. In order to answer the question, it is necessary to see the first realization of the two-parameter quantum toroidal algebra.

This paper is organized as follows. In section 2 the classical action of the squashed WZNW models is introduced. In section 3 we consider the classical integrable structure based on $S U(2)_{\mathrm{L}}$. This is called the left description. We present a couple of Lax pairs and the associated monodromy matrices. The classical $r / s$-matrices are shown to satisfy the extended classical Yang-Baxter equation. The infinite-dimensional extensions of $S U(2)_{\mathrm{L}}$ are Yangians. In section 4 we argue the classical integrable structure based on $U(1)_{\mathrm{R}}$. This is called the right description. A Lax pair and the associated monodromy matrix are presented. The classical $r / s$-matrices satisfy the extended classical Yang-Baxter equation. Remarkably, an infinite-dimensional extension of $U(1)_{\mathrm{R}}$ is shown to be a deformation of quantum affine algebra, where a new deformation parameter is provided by the coefficient of the WessZumino term. In section 5 the gauge equivalence between the left and right descriptions is proven. Under an identification between the spectral parameters, the left Lax pair is related to the right Lax pair via a gauge transformation. In section 6 we argue two degenerate limits in the right description. At some special points in the parameter space, the deformed quantum affine algebra degenerates to a Yangian, according to the enhancement of $U(1)_{\mathrm{R}}$ to $S U(2)_{\mathrm{R}}$. Section 7 is devoted to conclusion and discussion.

In Appendix A, we explain the computation of the current algebra in detail. Appendix B provides a prescription to treat non-ultra local terms in computing of the Poisson brackets of the conserved charges. 


\section{Preliminary}

Let us begin with the setup to fix our notation and convention. The metric of squashed $\mathrm{S}^{3}$ is first provided in terms of an $S U(2)$ group element. Then the classical action of the squashed WZNW models is introduced. For later convenience, the equations of motion are explicitly written down.

\subsection{Squashed $\mathrm{S}^{3}$}

The metric of round $\mathrm{S}^{3}$ can be described as a $U(1)$ fibration over $\mathrm{S}^{2}$. The squashing is one-parameter deformations of the $U(1)$ direction. The metric of squashed $\mathrm{S}^{3}$ is given by

$$
d s^{2}=\frac{L^{2}}{4}\left[d \theta^{2}+\sin ^{2} \theta d \phi^{2}+(1+C)(d \psi+\cosh \theta d \phi)^{2}\right]
$$

When $C=0$, the metric is reduced to that of $\mathrm{S}^{3}$ with radius $L$ and the isometry is $S U(2)_{\mathrm{L}} \times$ $S U(2)_{\mathrm{R}}$. When $C \neq 0$, the isometry is reduced to $S U(2)_{\mathrm{L}} \times U(1)_{\mathrm{R}}$.

In order to rewrite the metric (2.1), let us introduce an $S U(2)$ group element $g$ like

$$
g=\mathrm{e}^{T^{3} \phi} \mathrm{e}^{T^{2} \theta} \mathrm{e}^{T^{3} \psi} \in S U(2)
$$

Here the $s u(2)$ generators $T^{a}(a=1,2,3)$ satisfy the following relations

$$
\left[T^{a}, T^{b}\right]=\varepsilon^{a b}{ }_{c}^{c}
$$

and normalized as

$$
\operatorname{Tr}\left(T^{a} T^{b}\right)=-\frac{1}{2} \delta^{a b}
$$

Note that $\varepsilon^{a b}{ }_{c}$ is the totally anti-symmetric tensor normalized as $\varepsilon_{123}=+1$. The $\operatorname{su}(2)$ indices are raised and lowered by $\delta^{a b}$ and its inverse, respectively.

It is useful to define $T^{ \pm}$as

$$
T^{ \pm} \equiv \frac{1}{\sqrt{2}}\left(T^{1} \pm i T^{2}\right) .
$$

Then the commutation relations in (2.3) are rewritten as

$$
\left[T^{ \pm}, T^{\mp}\right]=\mp i T^{3}, \quad\left[T^{ \pm}, T^{3}\right]= \pm i T^{ \pm}
$$


and the normalization of the generators in (2.4) is given by

$$
\operatorname{Tr}\left(T^{ \pm} T^{\mp}\right)=\operatorname{Tr}\left(T^{3} T^{3}\right)=-\frac{1}{2} .
$$

Then the metric (2.1) is rewritten in terms of the $S U(2)$ group element as

$$
d s^{2}=-\frac{L^{2}}{2}\left[\operatorname{Tr}\left(J^{2}\right)-2 C\left(\operatorname{Tr}\left[T^{3} J\right]\right)^{2}\right]
$$

where we have introduced the left-invariant one-form

$$
J \equiv g^{-1} d g
$$

Note that $J$ can be represented by the angle variables $(\theta, \phi, \psi)$ as follows:

$$
\begin{aligned}
J & =T^{1}(\sin \psi d \theta-\cos \psi \sin \theta d \phi)+T^{2}(\cos \psi d \theta+\sin \psi \sin \theta d \phi)+T^{3}(d \psi+\cos \theta d \phi) \\
& =T^{+} \frac{1}{\sqrt{2}} \mathrm{e}^{i \psi}(-i d \theta-\sin \theta d \phi)+T^{-} \frac{1}{\sqrt{2}} \mathrm{e}^{-i \psi}(i d \theta-\sin \theta d \phi)+T^{3}(d \psi+\cos \theta d \phi)
\end{aligned}
$$

With the metric (2.8), it is easy to see the invariance under $S U(2)_{\mathrm{L}} \times U(1)_{\mathrm{R}}$. The $S U(2)_{\mathrm{L}}$ and $U(1)_{\mathrm{R}}$ transformations are the left- and right- multiplications,

$$
g \rightarrow \mathrm{e}^{\beta_{a} T^{a}} \cdot g \cdot \mathrm{e}^{-\alpha T^{3}}
$$

Here $\beta_{a}$ and $\alpha$ are real parameters.

\subsection{The classical action of the squashed WZNW models}

First of all, let us introduce two-dimensional non-linear sigma models whose target space is given by squashed $\mathrm{S}^{3}$. The classical action is

$$
S_{\sigma M}=\frac{1}{\lambda^{2}} \int_{-\infty}^{\infty} d t \int_{-\infty}^{\infty} d x \eta^{\mu \nu}\left[\operatorname{tr}\left(J_{\mu} J_{\nu}\right)-2 C \operatorname{tr}\left(T^{3} J_{\mu}\right) \operatorname{tr}\left(T^{3} J_{\nu}\right)\right]
$$

where the parameter $\lambda$ is the coupling constant and the base space is a two-dimensional Minkowski spacetime with the coordinates $t$ (time) and $x$ (space) and the metric

$$
-\eta_{t t}=\eta_{x x}=+1
$$

Note that the region of the parameter $C$ is restricted $C>-1$ so that the positivity of the kinetic term is ensured. 
The next is to introduce the Wess-Zumino term on squashed $\mathrm{S}^{3}$,

$$
S_{W Z}=\frac{n}{12 \pi} \int_{0}^{1} d s \int_{-\infty}^{\infty} d t \int_{-\infty}^{\infty} d x \epsilon^{\hat{\mu} \hat{\nu} \hat{\rho}} \operatorname{tr}\left(\widetilde{J}_{\hat{\mu}} \widetilde{J}_{\hat{\nu}} \widetilde{J}_{\hat{\rho}}\right), \quad \widetilde{J} \equiv \widetilde{g}^{-1} d \widetilde{g}
$$

where $n$ is an integer. Note that the above integral is performed on a three-dimensional base manifold spanned by $(t, x, s)$. The totally anti-symmetric tensor $\epsilon_{\hat{\mu} \hat{\nu} \hat{\rho}}$ is normalized as

$$
\epsilon_{t x s}=+1
$$

The $S U(2)$ element $\widetilde{g}$ is defined on this three-dimensional manifold. It interporates between a constant element at $s=0$ and $g(t, x)$ at $s=1$ :

$$
\widetilde{g}(t, x, s=0)=g_{0}: \text { const. }, \quad \widetilde{g}(t, x, s=1)=g(t, x) .
$$

Note that the Wess-Zumino term (2.13) is the same as in the case of round $\mathrm{S}^{3}$ and hence it is invariant under the $S U(2)_{\mathrm{L}} \times U(1)_{\mathrm{R}}$ symmetry of the sigma model action (2.11) .

Let us consider the Wess-Zumino-Novikov-Witten models defined on squashed $\mathrm{S}^{3}$, which henceforth are called "squashed WZNW models". The action is given by the sum of $S_{\sigma M}$ in (2.11) and $S_{W Z}$ in (2.13) :

$$
S=S_{\sigma M}+S_{W Z}
$$

The action (2.16) is also $S U(2)_{\mathrm{L}} \times U(1)_{\mathrm{R}}$-invariant.

From the action (2.16), the equations of motion are obtained,

$$
\partial^{\mu} J_{\mu}-2 C \operatorname{tr}\left(T^{3} \partial^{\mu} J_{\mu}\right) T^{3}-2 C \operatorname{tr}\left(T^{3} J_{\mu}\right)\left[J^{\mu}, T^{3}\right]+K \epsilon^{\mu \nu} \partial_{\mu} J_{\nu}=0,
$$

where the new constant $K$ is defined as

$$
K \equiv \frac{n \lambda^{2}}{8 \pi}
$$

and the totally anti-symmetric tensor $\epsilon_{\mu \nu}$ is normalized as

$$
\epsilon_{t x}=+1
$$

The $s u(2)$ components of the left-invariant one-form $J^{a}$ are defined as

$$
J^{a} \equiv-2 \operatorname{Tr}\left(T^{a} J\right)
$$


or equivalently

$$
J=T^{+} J^{-}+T^{-} J^{+}+T^{3} J^{3} .
$$

In terms of $J^{a}$, the equations of motion are rewritten as

$$
\begin{aligned}
& (1+C) \partial^{\mu} J_{\mu}^{3}+K \epsilon^{\mu \nu} \partial_{\mu} J_{\nu}^{3}=0, \\
& \partial^{\mu} J_{\mu}^{ \pm} \mp i C J_{\mu}^{3} J^{ \pm, \mu}+K \epsilon^{\mu \nu} \partial_{\mu} J_{\nu}^{ \pm}=0 .
\end{aligned}
$$

By definition, the left-invariant one-form $J$ satisfies the flatness condition:

$$
\epsilon^{\mu \nu}\left(\partial_{\mu} J_{\nu}+J_{\mu} J_{\nu}\right)=0
$$

This condition can also be rewritten in terms of the components $J^{a}$ as

$$
\begin{aligned}
& \epsilon^{\mu \nu}\left(\partial_{\mu} J_{\nu}^{3}+i J_{\mu}^{+} J_{\nu}^{-}\right)=0 \\
& \epsilon^{\mu \nu}\left(\partial_{\mu} J_{\nu}^{ \pm} \pm i J_{\mu}^{3} J_{\nu}^{ \pm}\right)=0 .
\end{aligned}
$$

The flatness condition (2.25) enables us to rewrite the equations of motion (2.23) as

$$
\begin{aligned}
& (1+C) \partial^{\mu} J_{\mu}^{3}-i K \epsilon^{\mu \nu} J_{\mu}^{+} J_{\nu}^{-}=0, \\
& \partial^{\mu} J_{\mu}^{ \pm} \mp i C J_{\mu}^{3} J^{ \pm, \mu} \mp i K \epsilon^{\mu \nu} J_{\mu}^{3} J_{\nu}^{ \pm}=0 .
\end{aligned}
$$

The expressions in (2.25) play an important role in our later discussion.

\section{The left description}

In this section, we discuss the classical integrable structure of squashed WZNW models based on the $S U(2)_{\mathrm{L}}$ symmetry. We call it left description. This part contains a short review of the previous work [24].

First of all, we construct an $S U(2)_{\mathrm{L}}$ conserved current which satisfies the flatness condition. With the flat and conserved current, we obtain a Lax pair and the corresponding monodromy matrix. Then we compute the classical $r / s$-matrices for the Lax pair. Finally, we show that the $S U(2)_{\mathrm{L}}$ symmetry is enhanced to the Yangian algebra $\mathcal{Y}\left(s u(2)_{\mathrm{L}}\right)$. 


\subsection{Lax pairs}

The classical action (2.16) has the $S U(2)_{\mathrm{L}}$ symmetry and the associated conserved current is given by

$$
j_{\mu}^{L}=g J_{\mu} g^{-1}-2 C \operatorname{tr}\left(T^{3} J_{\mu}\right) g T^{3} g^{-1}-K \epsilon_{\mu \nu} g J^{\nu} g^{-1}
$$

The conservation law of this current is equivalent to the equations of motion in (2.17) like

$$
\begin{aligned}
\partial^{\mu} j_{\mu}^{L} & =g\left[\partial^{\mu} J_{\mu}-2 C \operatorname{tr}\left(T^{3} \partial^{\mu} J_{\mu}\right) T^{3}-2 C \operatorname{tr}\left(T^{3} J_{\mu}\right)\left[J^{\mu}, T^{3}\right]+K \epsilon^{\mu \nu} \partial_{\mu} J_{\nu}\right] g^{-1} \\
& =0
\end{aligned}
$$

Note that $j_{\mu}^{L}$ does not satisfy the flatness condition due to the deformation.

One may consider to improve $j_{\mu}^{L}$ so as to satisfy the flatness condition. This requirement leaves two improved currents [24],

$$
j_{\mu}^{L_{ \pm}}=g J_{\mu} g^{-1}-2 C \operatorname{tr}\left(T^{3} J_{\mu}\right) g T^{3} g^{-1}-K \epsilon_{\mu \nu} g J^{\nu} g^{-1} \mp A \epsilon_{\mu \nu} \partial^{\nu}\left(g T^{3} g^{-1}\right)
$$

with the coefficient $A$ represented by

$$
A=\sqrt{C\left(1-\frac{K^{2}}{1+C}\right)} .
$$

The subscripts \pm denote the degeneracy of the improved currents. The improved currents satisfy the following flatness condition,

$$
\epsilon^{\mu \nu}\left(\partial_{\mu} j_{\nu}^{L_{ \pm}}-j_{\mu}^{L_{ \pm}} j_{\nu}^{L_{ \pm}}\right)=0
$$

When $K=0, A=\sqrt{C}$ and the improved currents constructed in [22] are reproduced.

With the flat $S U(2)_{\mathrm{L}}$ currents, two Lax pairs are constructed as

$$
\begin{aligned}
& L_{t}^{L_{ \pm}}\left(x ; \lambda_{L_{ \pm}}\right)=\frac{1}{2}\left[L_{+}^{L_{ \pm}}\left(x ; \lambda_{L_{ \pm}}\right)+L_{-}^{L_{ \pm}}\left(x ; \lambda_{L_{ \pm}}\right)\right] \\
& L_{x}^{L_{ \pm}}\left(x ; \lambda_{L_{ \pm}}\right)=\frac{1}{2}\left[L_{+}^{L_{ \pm}}\left(x ; \lambda_{L_{ \pm}}\right)-L_{-}^{L_{ \pm}}\left(x ; \lambda_{L_{ \pm}}\right)\right] \\
& L_{+}^{L_{ \pm}}\left(x ; \lambda_{L_{ \pm}}\right)=\frac{1}{1+\lambda_{L_{ \pm}}} j_{+}^{L_{ \pm}}, \quad L_{-}^{L_{ \pm}}\left(x ; \lambda_{L_{ \pm}}\right)=\frac{1}{1-\lambda_{L_{ \pm}}} j_{-}^{L_{ \pm}} .
\end{aligned}
$$

Here $\lambda_{L_{ \pm}}$are spectral parameters and $j_{+}^{L_{ \pm}}$and $j_{-}^{L_{ \pm}}$are defined as

$$
j_{+}^{L_{ \pm}} \equiv j_{t}^{L_{ \pm}}+j_{x}^{L_{ \pm}}, \quad j_{-}^{L_{ \pm}} \equiv j_{t}^{L_{ \pm}}-j_{x}^{L_{ \pm}}
$$


The following commutation relation

$$
\left[\partial_{t}-L_{t}^{L_{ \pm}}\left(x ; \lambda_{L_{ \pm}}\right), \partial_{x}-L_{x}^{L_{ \pm}}\left(x ; \lambda_{L_{ \pm}}\right)\right]=0
$$

gives rise to the conservation law of the flat $S U(2)_{\mathrm{L}}$ current (equivalently, the equations of motion) and the flatness condition.

Then the monodromy matrices are defined as

$$
U^{L_{ \pm}}\left(\lambda_{L_{ \pm}}\right) \equiv \mathrm{P} \exp \left[\int_{-\infty}^{\infty} d x L_{x}^{L_{ \pm}}\left(x ; \lambda_{L_{ \pm}}\right)\right] .
$$

The symbol P denotes the path ordering. Due to the flatness of the Lax pairs in (3.7), the monodromy matrices are conserved,

$$
\frac{d}{d t} U^{L_{ \pm}}\left(\lambda_{L_{ \pm}}\right)=0
$$

Thus an infinite set of conserved charges can be obtained by expanding the monodromy matrices with respect to $\lambda_{L_{ \pm}}$around appropriate points. For example, the monodromy matrices can be expanded around $\lambda_{L_{ \pm}}=\infty$ as

$$
U^{L_{ \pm}}\left(\lambda_{L_{ \pm}}\right)=\exp \left[\sum_{n=0}^{\infty} \lambda_{L_{ \pm}}^{-n-1} Q_{(n)}^{L_{ \pm}}\right] .
$$

In the next subsection, we will discuss the algebra generated by $Q_{(n)}^{L_{ \pm}}$.

Before closing this subsection, let us discuss the $r / s$-matrices computed from the Lax pairs in (3.5) by following the Maillet formalism [35]. One can read off them from the Poisson brackets between the spatial components of the Lax pairs,

$$
\begin{aligned}
& \left\{L_{x}^{L_{ \pm}}\left(x ; \lambda_{L_{ \pm}}\right) \stackrel{\otimes}{,} L_{x}^{L_{ \pm}}\left(y ; \mu_{L_{ \pm}}\right)\right\}_{\mathrm{P}} \\
= & {\left[r^{L_{ \pm}}\left(\lambda_{L_{ \pm}}, \mu_{L_{ \pm}}\right), L_{x}^{L_{ \pm}}\left(x ; \lambda_{L_{ \pm}}\right) \otimes 1+1 \otimes L_{x}^{L_{ \pm}}\left(y ; \mu_{L_{ \pm}}\right)\right] \delta(x-y) } \\
& -\left[s^{L_{ \pm}}\left(\lambda_{L_{ \pm}}, \mu_{L_{ \pm}}\right), L_{x}^{L_{ \pm}}\left(x ; \lambda_{L_{ \pm}}\right) \otimes 1-1 \otimes L_{x}^{L_{ \pm}}\left(y ; \mu_{L_{ \pm}}\right)\right] \delta(x-y) \\
& -2 s^{L_{ \pm}}\left(\lambda_{L_{ \pm}}, \mu_{L_{ \pm}}\right) \partial_{x} \delta(x-y) .
\end{aligned}
$$

To compute the above Poisson bracket, we have to use the current algebra for $j_{\mu}^{L_{ \pm}}$,

$$
\begin{aligned}
& \left\{j_{t}^{L_{ \pm}, a}(x), j_{t}^{L_{ \pm}, b}(y)\right\}_{\mathrm{P}}=\varepsilon^{a b}{ }_{c} j_{t}^{L_{ \pm}, c}(x) \delta(x-y)-2 K \delta^{a b} \partial_{x} \delta(x-y), \\
& \left\{j_{t}^{L_{ \pm}, a}(x), j_{x}^{L_{ \pm}, b}(y)\right\}_{\mathrm{P}}=\varepsilon^{a b}{ }_{c} j_{x}^{L_{ \pm}, c}(x) \delta(x-y)+\left(1+K^{2}+A^{2}\right) \delta^{a b} \partial_{x} \delta(x-y),
\end{aligned}
$$




$$
\begin{aligned}
\left\{j_{x}^{L_{ \pm}, a}(x), j_{x}^{L_{ \pm}, b}(y)\right\}_{\mathrm{P}}= & -\left(K^{2}+A^{2}\right) \varepsilon^{a b}{ }_{c} j_{t}^{L_{ \pm}, c}(x) \delta(x-y) \\
& -2 K \varepsilon^{a b}{ }_{c} j_{x}^{L_{ \pm}, c}(x) \delta(x-y)-2 K \delta^{a b} \partial_{x} \delta(x-y) .
\end{aligned}
$$

The explicit expressions of $r / s$-matrices are given by

$$
\begin{aligned}
& r^{L_{ \pm}}\left(\lambda_{ \pm}, \mu_{ \pm}\right)=\frac{h_{C, K}^{L}\left(\lambda_{ \pm}\right)+h_{C, K}^{L}\left(\mu_{ \pm}\right)}{2\left(\lambda_{ \pm}-\mu_{ \pm}\right)}\left[T^{-} \otimes T^{+}+T^{+} \otimes T^{-}+T^{3} \otimes T^{3}\right] \\
& s^{L_{ \pm}}\left(\lambda_{ \pm}, \mu_{ \pm}\right)=\frac{h_{C, K}^{L}\left(\lambda_{ \pm}\right)-h_{C, K}^{L}\left(\mu_{ \pm}\right)}{2\left(\lambda_{ \pm}-\mu_{ \pm}\right)}\left[T^{-} \otimes T^{+}+T^{+} \otimes T^{-}+T^{3} \otimes T^{3}\right]
\end{aligned}
$$

where a scalar function $h_{C, K}^{L}(\lambda)$ is defined as

$$
h_{C, K}^{L}(\lambda) \equiv \frac{A^{2}+(\lambda+K)^{2}}{1-\lambda^{2}} .
$$

The $r / s$-matrices satisfy the extended classical Yang-Baxter equation*,

$$
\begin{aligned}
& {\left[(r-s)_{12}^{L_{ \pm}}\left(\lambda_{L_{ \pm}}, \mu_{L_{ \pm}}\right),(r+s)_{13}^{L_{ \pm}}\left(\lambda_{L_{ \pm}}, \nu_{L_{ \pm}}\right)\right] } \\
+ & {\left[(r+s)_{12}^{L_{ \pm}}\left(\lambda_{L_{ \pm}}, \mu_{L_{ \pm}}\right),(r+s)_{23}^{L_{ \pm}}\left(\mu_{L_{ \pm}}, \nu_{L_{ \pm}}\right)\right] } \\
+ & {\left[(r+s)_{13}^{L_{ \pm}}\left(\lambda_{L_{ \pm}}, \nu_{L_{ \pm}}\right),(r+s)_{23}^{L_{ \pm}}\left(\mu_{L_{ \pm}}, \nu_{L_{ \pm}}\right)\right]=0 . }
\end{aligned}
$$

It should be noted that, when $K=0$, the function $l_{C, K}^{L}(\lambda)$ is reduced to

$$
h_{C, 0}^{L}(\lambda) \equiv \frac{C+\lambda^{2}}{1-\lambda^{2}}
$$

Thus the $r / s$-matrices in (3.13) reproduce the results without the Wess-Zumino term [25] .

\subsection{Yangians}

So far, the monodromy matrices $U^{L_{ \pm}}\left(\lambda_{L_{ \pm}}\right)$have been introduced and an infinite number of the conserved charges $Q_{(n)}^{L_{ \pm}}$are obtained by expanding them with respect to $\lambda_{L_{ \pm}}$.

For the first three levels, the explicit expressions of $Q_{(n)}^{L_{ \pm}}$are given by

$$
\begin{aligned}
Q_{(0)}^{L_{ \pm}, a} & =\int_{-\infty}^{\infty} d x j_{t}^{L_{ \pm}, a}(x) \\
Q_{(1)}^{L_{ \pm}, a} & =\frac{1}{4} \int_{-\infty}^{\infty} d x \int_{-\infty}^{\infty} d y \epsilon(x-y) \varepsilon^{a}{ }_{b c} j_{t}^{L_{ \pm}, b}(x) j_{t}^{L_{ \pm}, c}(y)-\int_{-\infty}^{\infty} j_{x}^{L_{ \pm}, a}(x)
\end{aligned}
$$

* The $r / s$-matrices depend on $\lambda_{L_{ \pm}}$and $\mu_{L_{ \pm}}$individually (not only $\lambda_{L_{ \pm}}-\mu_{L_{ \pm}}$) and they satisfy the extended classical Yang-Baxter equation. Thus the classification of the $r / s$-matrice are subtle. 


$$
\begin{aligned}
Q_{(2)}^{L_{ \pm}, a}= & \frac{1}{12} \int_{-\infty}^{\infty} d x \int_{-\infty}^{\infty} d y \int_{-\infty}^{\infty} d z \epsilon(x-y) \epsilon(x-z) \delta_{b c} \\
& \times\left[j_{t}^{L_{ \pm}, b}(x) j_{t}^{L_{ \pm}, a}(y) j_{t}^{L_{ \pm}, c}(z)-j_{t}^{L_{ \pm}, a}(x) j_{t}^{L_{ \pm}, b}(y) j_{t}^{L_{ \pm}, c}(z)\right] \\
& -\frac{1}{2} \int_{-\infty}^{\infty} d x \int_{-\infty}^{\infty} d y \epsilon(x-y) \varepsilon_{b c}^{a} j_{t}^{L_{ \pm}, b}(x) j_{x}^{L_{ \pm}, c}(y)+\int_{-\infty}^{\infty} d x j_{t}^{L_{ \pm}, a}(x) .
\end{aligned}
$$

Note that these charges can be directly constructed from the flat $S U(2)_{\mathrm{L}}$ currents $j_{\mu}^{L_{ \pm}}(x)$ recursively by following the BIZZ construction [8] .

The next task is to show that the conserved charges satisfy the defining relations of Yangian $\mathcal{Y}\left(s u(2)_{\mathrm{L}}\right)$. The Poisson brackets of the first two levels are given by [24]

$$
\begin{aligned}
& \left\{Q_{(0)}^{L_{ \pm}, a}, Q_{(0)}^{L_{ \pm}, b}\right\}_{\mathrm{P}}=\varepsilon^{a b}{ }_{c} Q_{(0)}^{L_{ \pm}, c}, \\
& \left\{Q_{(1)}^{L_{ \pm}, a}, Q_{(0)}^{L_{ \pm}, b}\right\}_{\mathrm{P}}=\varepsilon^{a b}{ }_{c} Q_{(1)}^{L_{ \pm}, c}, \\
& \left\{Q_{(1)}^{L_{ \pm}, a}, Q_{(1)}^{L_{ \pm}, b}\right\}_{\mathrm{P}}=\varepsilon^{a b}{ }_{c}\left[Q_{(2)}^{L_{ \pm}, c}+\frac{1}{12}\left(Q_{(0)}^{L_{ \pm}}\right)^{2} Q_{(0)}^{L_{ \pm}, c}+2 K Q_{(1)}^{L_{ \pm}, c}\right] .
\end{aligned}
$$

The Serre relations are shown as

$$
\begin{aligned}
& \left\{Q_{(1)}^{L_{ \pm}, 3},\left\{Q_{(1)}^{L_{ \pm},+}, Q_{(1)}^{L_{ \pm},-}\right\}_{\mathrm{P}}\right\}_{\mathrm{P}}=\frac{1}{4} Q_{(0)}^{L_{ \pm}, 3}\left(Q_{(0)}^{L_{ \pm},+} Q_{(1)}^{L_{ \pm},-}-Q_{(0)}^{L_{ \pm},-} Q_{(1)}^{L_{ \pm},+}\right), \\
& \left\{Q_{(1)}^{L_{ \pm}, \pm},\left\{Q_{(1)}^{L_{ \pm}, \pm}, Q_{(1)}^{L_{ \pm}, \mp}\right\}_{\mathrm{P}}\right\}_{\mathrm{P}}-\left\{Q_{(1)}^{L_{ \pm}, 3},\left\{Q_{(1)}^{L_{ \pm}, \pm}, Q_{(1)}^{L_{ \pm}, 3}\right\}_{\mathrm{P}}\right\}_{\mathrm{P}} \\
& =\frac{1}{4} Q_{(0)}^{L_{ \pm}, \pm}\left(Q_{(0)}^{L_{ \pm}, \pm} Q_{(1)}^{L_{ \pm}, \mp}-Q_{(0)}^{L_{ \pm}, \mp} Q_{(1)}^{L_{ \pm}, \pm}\right)-\frac{1}{4} Q_{(0)}^{L_{ \pm}, 3}\left(Q_{(0)}^{L_{ \pm}, \pm} Q_{(1)}^{L_{ \pm}, 3}-Q_{(0)}^{L_{ \pm}, 3} Q_{(1)}^{L_{ \pm}, \pm}\right), \\
& \left\{Q_{(1)}^{L_{ \pm}, \pm},\left\{Q_{(1)}^{L_{ \pm}, \pm}, Q_{(1)}^{L_{ \pm}, 3}\right\}_{\mathrm{P}}\right\}_{\mathrm{P}}=\frac{1}{4} Q_{(0)}^{L_{ \pm}, \pm}\left(Q_{(0)}^{L_{ \pm}, \pm} Q_{(1)}^{L_{ \pm}, 3}-Q_{(0)}^{L_{ \pm}, 3} Q_{(1)}^{L_{ \pm}, \pm}\right) .
\end{aligned}
$$

Thus the defining relations of Yangian $\mathcal{Y}\left(s u(2)_{\mathrm{L}}\right)$ at the classical level are satisfied in the sense of Drinfeld's first realization [36, 37].

Here we should comment on the treatment of non-ultra local terms contained in the current algebra of $j_{\mu}^{L_{ \pm}}(x)$. They develop ambiguities in computing the Poisson brackets of the conserved charges and there might be the possibility that the defining relations of $\mathcal{Y}\left(s u(2)_{\mathrm{L}}\right)$ are spoiled. In the present case, the presence of the Wess-Zumino term make the situation worse. It develops non-ultra local terms even in the Poisson brackets of $j_{t}^{L_{ \pm}}(x)$ and hence cause ambiguities in computing the usual $S U(2)_{\mathrm{L}}$ Lie algebra part. The treatment of the non-ultra local terms is argued in Appendix B in detail. 


\section{The right description}

The classical integrable structure of the squashed WZNW models can also be described based on $U(1)_{\mathrm{R}}$ as another description. This description is called right description. A Lax pair and the associated monodromy matrix are presented. Then an infinite-dimensional extension of $U(1)_{\mathrm{R}}$ is argued. The resulting algebra is a deformation of the standard quantum affine algebra. In the right description, the $r / s$-matrices are deformed by an additional term, in comparison to the case without the Wess-Zumino term.

\subsection{Lax pair}

A Lax pair which respects $U(1)_{\mathrm{R}}$ is given by

$$
\begin{aligned}
& L_{t}^{R}\left(x ; \lambda_{R}\right)=\frac{1}{2}\left[L_{+}^{R}\left(x ; \lambda_{R}\right)+L_{-}^{R}\left(x ; \lambda_{R}\right)\right], \\
& L_{x}^{R}\left(x ; \lambda_{R}\right)=\frac{1}{2}\left[L_{+}^{R}\left(x ; \lambda_{R}\right)-L_{-}^{R}\left(x ; \lambda_{R}\right)\right], \\
& L_{ \pm}^{R}\left(x ; \lambda_{R}\right)=-\frac{\sinh (\alpha \pm \beta)}{\sinh \left[\alpha \pm\left(\beta+\lambda_{R}\right)\right]}\left[T^{+} J_{ \pm}^{-}+T^{-} J_{ \pm}^{+}+\frac{\cosh \left(\alpha \pm \lambda_{R}\right)}{\cosh \alpha} T^{3} J_{ \pm}^{3}\right] .
\end{aligned}
$$

New constants $\alpha$ and $\beta$ are related to $C$ and $K$ like

$$
\tanh \alpha=\frac{i C}{A}, \quad \tanh \beta=\frac{i C K}{A(1+C)} .
$$

Note that $\alpha$ and $\beta$ have the periodicities,

$$
\alpha \sim \alpha+\pi i, \quad \beta \sim \beta+\pi i .
$$

In the Lax pair (4.1), a spectral parameter $\lambda_{R}$ has been introduced. This is seemingly independent of $\lambda_{L_{ \pm}}$at this stage, but eventually there is a relation between them as we will see later. The Lax pair (4.1) is referred to as the right Lax pair hereafter.

The relations given in (4.2) imply the inequalities for $(C, K)$ :

$$
\begin{aligned}
-\tanh ^{2} \alpha & =\frac{C^{2}}{A^{2}}=\frac{C(1+C)}{1+C-K^{2}}>-1 \\
-\tanh ^{2} \beta & =\frac{C^{2} K^{2}}{A^{2}(1+C)^{2}}=\frac{C K^{2}}{(1+C)\left(1+C-K^{2}\right)}>-1 .
\end{aligned}
$$

With the kinematic restriction $C>-1$, these inequalities are equivalent to

$$
(C-K+1)(C+K+1)\left(C-K^{2}+1\right)>0 .
$$

\footnotetext{
${ }^{\dagger}$ The anisotropic Lax pair with $K=0$ is constructed originally by Cherednik [19]. See also [20].
} 


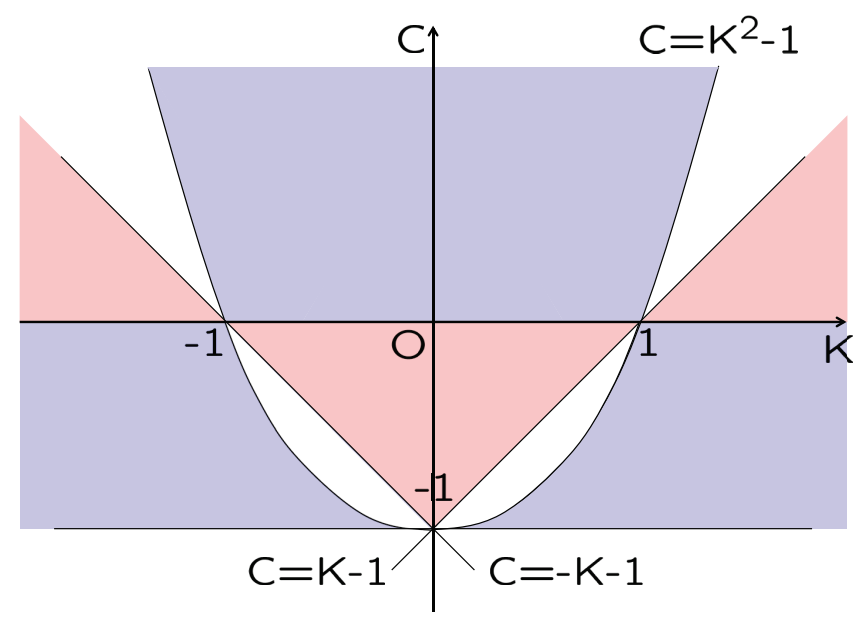

Figure 1: The allowed region of $C$ and $K$. In the red region, $\alpha$ and $\beta$ are real. On the other hand, these are purely imaginary in the blue region.

Due to the relations in (4.2) and the reality of $C$ and $K, \alpha$ and $\beta$ must be real or purely imaginary. When $C\left(C-K^{2}+1\right)<0, \alpha$ and $\beta$ are real (up to the shift of $i \pi n$ with $n \in \mathbb{Z}$ ). On the other hand, when $C\left(C-K^{2}+1\right)>0, \alpha$ and $\beta$ are purely imaginary. Thus the allowed region of $C$ and $K$ can be expressed on the $(C, K)$-plane as depicted in Fig.11.

The relations given in (4.2) can be solved for $\alpha$ and $\beta$, and hence $C, K$ (and $A$ ) are written in terms of $\alpha$ and $\beta$,

$$
\begin{aligned}
& K=\frac{\sinh 2 \beta}{\sinh 2 \alpha}, \quad C=-\frac{\sinh (\alpha+\beta) \sinh (\alpha-\beta)}{\cosh ^{2} \alpha}, \\
& A=\frac{-2 i \sinh (\alpha+\beta) \sinh (\alpha-\beta)}{\sinh 2 \alpha} .
\end{aligned}
$$

Note that $(C, K, A)$ are invariant under the shift of $\alpha$ and $\beta$ by $i \pi$.

The equations of motion in (2.17) and the flatness condition of $J$ are reproduced from the commutation relation,

$$
\left[\partial_{t}-L_{t}^{R}\left(x ; \lambda_{R}\right), \partial_{x}-L_{x}^{R}\left(x ; \lambda_{R}\right)\right]=0 .
$$

\section{Monodromy matrix}

With the right Lax pair given in (4.1), the associated monodromy matrix is defined as

$$
U^{R}\left(\lambda_{R}\right) \equiv \mathrm{P} \exp \left[\int_{-\infty}^{\infty} d x L_{x}^{R}\left(x ; \lambda_{R}\right)\right] .
$$


The flatness of the Lax pair (4.6) ensures that the monodromy matrix is a conserved quantity,

$$
\frac{d}{d t} U^{R}\left(\lambda_{R}\right)=0
$$

Thus, by expanding $U^{R}\left(\lambda_{R}\right)$, an infinite number of conserved charges are constructed.

\section{$4.2 q$-deformation of $s u(2)_{\mathrm{R}}$}

In the squashed WZNW models, the $S U(2)_{\mathrm{R}}$ symmetry, which is preserved by round $\mathrm{S}^{3}$, is broken to $U(1)_{\mathrm{R}}$, due to the deformation term. The Noether current for $U(1)_{\mathrm{R}}$ is given by

$$
\begin{aligned}
j_{\mu}^{R, 3} & =-\left[(1+C) J_{\mu}^{3}+K \epsilon_{\mu \nu} J^{3, \nu}\right] \\
& =-\frac{\cosh \beta}{\cosh \alpha}\left(\frac{\cosh \beta}{\cosh \alpha} J_{\mu}^{3}+\frac{\sinh \beta}{\sinh \alpha} \epsilon_{\mu \nu} J^{3, \nu}\right) .
\end{aligned}
$$

For later convenience, the expressions have been given in terms of $\alpha$ and $\beta$, as well as in terms of $C$ and $K$.

It is known that there exist non-local conserved currents which correspond to the broken components $T^{ \pm}$in the case without the Wess-Zumino term [25]. Let us show that this is the case even in the squashed WZNW models.

For this purpose, it is helpful to introduce a non-local function $\chi(x)$,

$$
\chi(x)=-\frac{1}{2} \int_{-\infty}^{\infty} d y \epsilon(x-y) j_{t}^{R, 3}(y) .
$$

This function satisfies the differential equation,

$$
\partial_{\mu} \chi=-\epsilon_{\mu \nu} j^{R, 3, \nu}=(1+C) \epsilon_{\mu \nu} J^{3, \nu}+K J_{\mu}^{3} .
$$

This relation ensures the conservation law of the non-local currents, as we will see later.

With $\chi(x)$, the conserved, non-local currents are constructed as

$$
\begin{aligned}
j_{\mu}^{R, \pm} & =\mathrm{e}^{\gamma^{ \pm}} i_{\mu}^{R, \pm} \\
i_{\mu}^{R, \pm} & =-\left(J_{\mu}^{ \pm}+(K \pm i A) \epsilon_{\mu \nu} J^{ \pm, \nu}\right) \\
& =-\frac{1}{\sinh 2 \alpha}\left[\sinh 2 \alpha J_{\mu}^{ \pm} \pm\left(\cosh 2 \alpha-\mathrm{e}^{\mp 2 \beta}\right) \epsilon_{\mu \nu} J^{ \pm, \nu}\right] .
\end{aligned}
$$

Here $\gamma^{ \pm}$are defined as

$$
\gamma^{ \pm} \equiv\left(\frac{1+C}{C} A \mp i K\right)^{-1}=-i \mathrm{e}^{ \pm \beta} \frac{\sinh 2 \alpha}{2 \cosh \beta} .
$$


The non-local currents $j_{\mu}^{R, \pm}$ give rise to the conserved charges

$$
Q^{R, \pm}=\int_{-\infty}^{\infty} d x j_{t}^{R, \pm}(x)
$$

as well as the standard Noether charge of $U(1)_{\mathrm{R}}$,

$$
Q^{R, 3}=\int_{-\infty}^{\infty} d x j_{t}^{R, 3}(x) .
$$

As a next step, let us compute the algebra of $Q^{R, \pm}$ and $Q^{R, 3}$. For this purpose, it is necessary to evaluate the Poisson brackets of $i_{t}^{R, \pm}(x)$ and $j_{t}^{R, 3}(x)$,

$$
\begin{aligned}
& \left\{j_{t}^{R, 3}(x), j_{t}^{R, 3}(y)\right\}_{\mathrm{P}}=2 K \partial_{x} \delta(x-y), \\
& \left\{i_{t}^{R, \pm}(x), j_{t}^{R, 3}(y)\right\}_{\mathrm{P}}= \pm i i_{t}^{R, \pm}(x) \delta(x-y), \\
& \left\{i_{t}^{R, \pm}(x), i_{t}^{R, \mp}(y)\right\}_{\mathrm{P}}=\mp i j_{t}^{R, 3}(x) \delta(x-y)+2 K \partial_{x} \delta(x-y) .
\end{aligned}
$$

Because the Poisson brackets contain non-ultra local terms even for the first bracket due to non-vanishing $K$, an appropriate prescription to treat the non-ultra local terms is required to evaluate the Poisson brackets of $Q^{R, \pm}$ and $Q^{R, 3}$, as in the case of the $s u(2)_{\mathrm{L}}$ algebra in the left description. The prescription is argued in Appendix B in detail.

The resulting algebra can be regarded as a classical analogue of $q$-deformed $s u(2)_{\mathrm{R}}$ [37,38] ,

$$
\begin{aligned}
& \left\{Q^{R, \pm}, Q^{R, 3}\right\}_{\mathrm{P}}= \pm i Q^{R, \pm} \\
& \left\{Q^{R,+}, Q^{R,-}\right\}_{\mathrm{P}}=-i \frac{\left(\gamma^{+}\right)^{2}+\left(\gamma^{-}\right)^{2}}{2 \gamma^{+} \gamma^{-}} \frac{2}{\left(\gamma^{+}+\gamma^{-}\right)} \sinh \left[\frac{\left(\gamma^{+}+\gamma^{-}\right)}{2} Q^{R, 3}\right] .
\end{aligned}
$$

The $q$-parameter is given by

$$
q=\mathrm{e}^{\left(\gamma^{+}+\gamma^{-}\right) / 2}=\mathrm{e}^{\gamma}
$$

where $\gamma$ is a new parameter defined as

$$
\gamma \equiv \frac{\gamma^{+}+\gamma^{-}}{2}=-\frac{i}{2} \sinh 2 \alpha
$$

Note that there is an unfamiliar ovarall factor $\left(\left(\gamma^{+}\right)^{2}+\left(\gamma^{-}\right)^{2}\right) / 2 \gamma^{+} \gamma^{-}$in the right-hand side of the Poisson bracket between $Q^{R,+}$ and $Q^{R,-}$. However, this factor can be absorbed by rescaling $Q^{R, \pm}$ without changing the Poisson structure. 
Similarly, there is another set of conserved, non-local currents,

$$
\begin{aligned}
\widetilde{j}_{\mu}^{R, \pm} & =\mathrm{e}^{-\gamma^{\mp} \chi_{i_{\mu}^{R}}^{R, \pm}}, \\
\widetilde{i}_{\mu}^{R, \pm} & =-\left(J_{\mu}^{ \pm}+(K \mp i A) \epsilon_{\mu \nu} J^{ \pm, \nu}\right) \\
& =-\frac{1}{\sinh 2 \alpha}\left[\sinh 2 \alpha J_{\mu}^{ \pm} \mp\left(\cosh 2 \alpha-\mathrm{e}^{ \pm 2 \beta}\right) \epsilon_{\mu \nu} J^{ \pm, \nu}\right],
\end{aligned}
$$

and the associated charges are

$$
\widetilde{Q}^{R, \pm}=\int_{-\infty}^{\infty} d x \widetilde{j}_{t}^{R, \pm}(x) .
$$

Note that $\widetilde{j}^{R, \pm}$ are related to $j_{\mu}^{R, \pm}(x)$ by flipping the sign of $A$. The sign flip of $A$ is translated to the following transformation laws,

$$
(\alpha, \beta) \rightarrow(-\alpha,-\beta), \quad \gamma^{ \pm} \rightarrow-\gamma^{\mp}
$$

Thus, with the sign flip of $A$, the algebra of $Q^{R, 3}$ and $\widetilde{Q}^{R, \pm}$ is obtained as

$$
\begin{aligned}
& \left\{\widetilde{Q}^{R, \pm}, Q^{R, 3}\right\}_{\mathrm{P}}= \pm i \widetilde{Q}^{R, \pm} \\
& \left\{\widetilde{Q}^{R,+}, \widetilde{Q}^{R,-}\right\}_{\mathrm{P}}=-i \frac{\left(\gamma^{+}\right)^{2}+\left(\gamma^{-}\right)^{2}}{2 \gamma^{+} \gamma^{-}} \frac{2}{\left(\gamma^{+}+\gamma^{-}\right)} \sinh \left[\frac{\left(\gamma^{+}+\gamma^{-}\right)}{2} Q^{R, 3}\right] .
\end{aligned}
$$

The algebra is also the $q$-deformed $s u(2)_{\mathrm{R}}$ with the same $q$-parameter.

\subsection{Expansions of the monodromy matrix}

The next task is to argue an infinite-dimensional extension of $q$-deformed $s u(2)_{\mathrm{R}}$. For this

purpose, let us consider two expansions of the monodromy matrix $U^{R}\left(\lambda_{R}\right)$ with respect to the spectral parameter $\lambda_{R}$. The expansion points are the same as in the case of squashed sigma models [26] and the monodromy matrix is expanded around $\operatorname{Re} \lambda_{R}= \pm \infty$. Note that the spectral parameter $\lambda_{R}$ is periodically identified as

$$
\lambda_{R} \sim \lambda_{R}+2 \pi i
$$

and hence the spectral parameter $\lambda_{R}$ is regarded as living on a cylinder. As a result, the point $\operatorname{Re} \lambda_{R}=+\infty$ is certainly different from the point $\operatorname{Re} \lambda_{R}=-\infty$. Thus the two expansion points give rise to two different sets of conserved charges. Here the expressions of the conserved charges are explicitly obtained. 
First of all, recall the concrete expression of $L_{x}^{R}\left(x ; \lambda_{R}\right)$ :

$$
\begin{aligned}
& L_{x}^{R}\left(x ; \lambda_{R}\right) \\
& =-\frac{1}{\sinh \left(\alpha+\beta+\lambda_{R}\right) \sinh \left(\alpha-\beta-\lambda_{R}\right)} \\
& \times\left\{T ^ { 3 } \left[-\frac{\sinh \alpha}{\cosh \alpha}\left(\cosh ^{2} \beta \cosh \lambda_{R} \sinh \lambda_{R}+\sinh \beta \cosh \beta \sinh ^{2} \lambda_{R}\right) J_{t}^{3}\right.\right. \\
& \quad+\quad \sinh (\alpha+\beta) \sinh (\alpha-\beta) \cosh ^{2} \lambda_{R} \\
& \left.\left.\quad-\sinh \beta \cosh \beta \cosh \lambda_{R} \sinh \lambda_{R}-\sinh ^{2} \alpha \sinh ^{2} \lambda_{R}\right) J_{x}^{3}\right] \\
& +T^{+}\left[-\sinh \alpha \cosh \alpha \sinh \lambda_{R} J_{t}^{-}\right. \\
& \left.\quad+\left(\sinh (\alpha+\beta) \sinh (\alpha-\beta) \cosh \lambda_{R}-\sinh \beta \cosh \beta \sinh \lambda_{R}\right) J_{x}^{-}\right] \\
& +\quad \sinh \alpha \cosh \alpha \sinh \lambda_{R} J_{t}^{+} \\
& \left.\left.\quad+\left(\sinh (\alpha+\beta) \sinh (\alpha-\beta) \cosh \lambda_{R}-\sinh \beta \cosh \beta \sinh \lambda_{R}\right) J_{x}^{+}\right]\right\} .
\end{aligned}
$$

This expression is useful to expand $U^{R}\left(\lambda_{R}\right)$.

\section{Expansion around $\operatorname{Re} \lambda_{R}=-\infty$}

Let us first expand $U^{R}\left(\lambda_{R}\right)$ around $\operatorname{Re} \lambda_{R}=-\infty$. It is convenient to perform a transformation for the spectral parameter as

$$
z_{R}=\mathrm{e}^{\lambda_{R}}
$$

The expansion around $\operatorname{Re} \lambda_{R}=-\infty$ corresponds to the one around $z_{R}=0$. Then the expanded monodromy matrix is expected to be the following form,

$$
U^{R}\left(\lambda_{R}\right)=\mathrm{e}^{q_{(0)} / 2} \exp \left[\sum_{n=1}^{\infty} z_{R}^{n} q_{(n)}\right] \mathrm{e}^{q_{(0)} / 2} .
$$

Here $q_{(n)}(n \geq 0)$ are conserved charges to be determined by a concrete expansion of $U^{R}\left(\lambda_{R}\right)$.

Note that $L_{x}^{R}\left(\lambda_{R}\right)$ is expanded around $z_{R}=0$ as

$$
\begin{aligned}
L_{x}^{R}\left(x ; \lambda_{R}\right)= & i \gamma^{+} T^{3} j_{t}^{R, 3}-z_{R} \mathrm{e}^{2 \beta} \sinh 2 \alpha\left(T^{-} i_{t}^{R,+}+T^{+} \widetilde{i}_{t}^{R,-}\right) \\
& -z_{R}^{2} \mathrm{e}^{2 \beta} \sinh ^{2} 2 \alpha T^{3}\left(j_{x}^{R, 3}+\frac{\cosh 2 \alpha}{\sinh 2 \alpha} j_{t}^{R, 3}\right) \\
& -z_{R}^{3} \mathrm{e}^{4 \beta} \sinh ^{2} 2 \alpha\left[T^{-}\left(i_{x}^{R,+}+\frac{\cosh 2 \alpha}{\sinh 2 \alpha} i_{t}^{R,+}\right)+T^{+}\left(\widetilde{i}_{x}^{R,-}+\frac{\cosh 2 \alpha}{\sinh 2 \alpha} \widetilde{i}_{t}^{R,-}\right)\right]
\end{aligned}
$$




$$
+\mathcal{O}\left(z_{R}^{4}\right) .
$$

By comparing the expansion of the expected form (4.27)

$$
\begin{aligned}
U^{R}\left(\lambda_{R}\right)=\mathrm{e}^{q_{(0)} / 2}[ & 1+z_{R} q_{(1)}+z_{R}^{2}\left(q_{(2)}+\frac{1}{2} q_{(1)}^{2}\right) \\
& \left.+z_{R}^{3}\left(q_{(3)}+\frac{1}{2}\left\{q_{(1)}, q_{(2)}\right\}+\frac{1}{6} q_{(1)}^{3}\right)+\mathcal{O}\left(z_{R}^{4}\right)\right] \mathrm{e}^{q_{(0)} / 2},
\end{aligned}
$$

with the direct expansion of $U^{R}\left(\lambda_{R}\right)$ using (4.28), the expressions of $q_{(n)}$ are fixed as follows:

$$
\begin{aligned}
& q_{(0)}=i \gamma^{+} T^{3} Q_{(0)}^{R, 3}, \\
& q_{(1)}=-\mathrm{e}^{2 \beta} \sinh 2 \alpha\left(T^{-} Q_{(1)}^{R,+}+T^{+} \widetilde{Q}_{(1)}^{R,-}\right), \\
& q_{(2)}=-\frac{i}{2} \mathrm{e}^{4 \beta} \sinh ^{2} 2 \alpha T^{3} Q_{(2)}^{R, 3}, \\
& q_{(3)}=-\frac{1}{4} \mathrm{e}^{6 \beta} \sinh ^{3} 2 \alpha\left(T^{-} Q_{(3)}^{R,+}+T^{+} \widetilde{Q}_{(3)}^{R,-}\right) .
\end{aligned}
$$

where the following quantities have been introduced,

$$
\begin{aligned}
& Q_{(0)}^{R, 3}=\int_{-\infty}^{\infty} d x j_{t}^{R, 3}(x) \quad\left(=Q^{R, 3}\right), \\
& Q_{(1)}^{R,+}=\int_{-\infty}^{\infty} d x j_{t}^{R,+}(x) \quad\left(=Q^{R,+}\right), \quad \widetilde{Q}_{(1)}^{R,-}=\int_{-\infty}^{\infty} d x \widetilde{j}_{t}^{R,-}(x) \quad\left(=\widetilde{Q}^{R,-}\right), \\
& Q_{(2)}^{R, 3}=-\int_{-\infty}^{\infty} d x \int_{-\infty}^{\infty} d y \epsilon(x-y) j_{t}^{R,+}(x) \widetilde{j}_{t}^{R,-}(y) \\
& -2 i \mathrm{e}^{-2 \beta} \int_{-\infty}^{\infty} d x j_{x}^{R, 3}(x)-2 i \mathrm{e}^{-2 \beta} \frac{\cosh 2 \alpha}{\sinh 2 \alpha} Q_{(0)}^{R, 3}, \\
& Q_{(3)}^{R,+}=\frac{1}{2} \int_{-\infty}^{\infty} d x \int_{-\infty}^{\infty} d y \int_{-\infty}^{\infty} d z \epsilon(x-y) \epsilon(x-z) \widetilde{j}_{t}^{R,-}(x) j_{t}^{R,+}(y) j_{t}^{R,+}(z) \\
& +2 i \mathrm{e}^{-2 \beta} \int_{-\infty}^{\infty} d x \int_{-\infty}^{\infty} d y \epsilon(x-y) j_{t}^{R,+}(x)\left(j_{x}^{R, 3}(y)+\frac{\cosh 2 \alpha}{\sinh 2 \alpha} j_{t}^{R, 3}(y)\right) \\
& +\frac{4 \mathrm{e}^{-2 \beta}}{\sinh 2 \alpha} \int_{-\infty}^{\infty} d x j_{x}^{R,+}(x)-\frac{1}{6} \widetilde{Q}_{(1)}^{R,+}\left(Q_{(1)}^{R,+}\right)^{2}+\frac{4 \mathrm{e}^{-2 \beta} \cosh 2 \alpha}{\sinh ^{2} 2 \alpha} Q_{(1)}^{R,+}, \\
& \widetilde{Q}_{(3)}^{R,-}=\frac{1}{2} \int_{-\infty}^{\infty} d x \int_{-\infty}^{\infty} d y \int_{-\infty}^{\infty} d z \epsilon(x-y) \epsilon(x-z) j_{t}^{R,+}(x) \widetilde{j}_{t}^{R,-}(y) \widetilde{j}_{t}^{R,-}(z) \\
& -2 i \mathrm{e}^{-2 \beta} \int_{-\infty}^{\infty} d x \int_{-\infty}^{\infty} d y \epsilon(x-y) \widetilde{j}_{t}^{R,-}(x)\left(j_{x}^{R, 3}(y)+\frac{\cosh 2 \alpha}{\sinh 2 \alpha} j_{t}^{R, 3}(y)\right) \\
& +\frac{4 \mathrm{e}^{-2 \beta}}{\sinh 2 \alpha} \int_{-\infty}^{\infty} d x \widetilde{j}_{x}^{R,-}(x)-\frac{1}{6} Q_{(1)}^{R,+}\left(\widetilde{Q}_{(1)}^{R,-}\right)^{2}+\frac{4 \mathrm{e}^{-2 \beta} \cosh 2 \alpha}{\sinh ^{2} 2 \alpha} \widetilde{Q}_{(1)}^{R,-} .
\end{aligned}
$$


These are also conserved charges and will play an important role later in studying the tower structure of the conserved charges that indicates an infinite-dimensional extension of $q$-deformed $s u(2)_{\mathrm{R}}$.

\section{Expansion around $\operatorname{Re} \lambda_{R}=+\infty$}

Let us next consider another expansion around $\operatorname{Re} \lambda_{R}=+\infty$. It is helpful to introduce a new parametrization $z_{R}^{\prime}$ defined as

$$
z_{R}^{\prime}=z_{R}^{-1}=\mathrm{e}^{-\lambda_{R}}
$$

The expansion around $\operatorname{Re} \lambda_{R}=+\infty$ corresponds to the one around $z_{R}^{\prime}=0$. Then the expected form of the expanded monodromy matrix $U^{R}\left(\lambda_{R}\right)$ is given by

$$
U^{R}\left(\lambda_{R}\right)=\mathrm{e}^{\bar{q}_{(0)} / 2} \exp \left[\sum_{n=1}^{\infty} z_{R}^{\prime n} \bar{q}_{(n)}\right] \mathrm{e}^{\bar{q}_{(0)} / 2} .
$$

Here $\bar{q}_{(n)}(n \geq 0)$ are conserved charges.

The Lax pair $L_{x}^{R}\left(\lambda_{R}\right)$ is expanded around $z_{R}^{\prime}=0$ as

$$
\begin{aligned}
L_{x}^{R}\left(x ; \lambda_{R}\right)= & i \gamma^{-} T^{3} j_{t}^{R, 3}+z_{R}^{\prime} \mathrm{e}^{-2 \beta} \sinh 2 \alpha\left(T^{+} i_{t}^{R,-}+T^{-} \widetilde{i}_{t}^{R,+}\right) \\
& -z_{R}^{\prime 2} \mathrm{e}^{-2 \beta} \sinh ^{2} 2 \alpha T^{3}\left(j_{x}^{R, 3}-\frac{\cosh 2 \alpha}{\sinh 2 \alpha} j_{t}^{R, 3}\right) \\
& -z_{R}^{\prime 3} \mathrm{e}^{-4 \beta} \sinh ^{2} 2 \alpha\left[T^{+}\left(i_{x}^{R,-}-\frac{\cosh 2 \alpha}{\sinh 2 \alpha} i_{t}^{R,-}\right)+T^{-}\left(\widetilde{i}_{x}^{R,+}-\frac{\cosh 2 \alpha}{\sinh 2 \alpha} i_{t}^{R,+}\right)\right] \\
& +\mathcal{O}\left(z_{R}^{\prime 4}\right) .
\end{aligned}
$$

Thus, by comparing the expansion of the expected monodromy matrix

$$
\begin{aligned}
U^{R}\left(\lambda_{R}\right)=\mathrm{e}^{\bar{q}_{(0)} / 2}[1 & +z_{R}^{\prime} \bar{q}_{(1)}+z_{R}^{\prime 2}\left(\bar{q}_{(2)}+\frac{1}{2} \bar{q}_{(1)}^{2}\right) \\
& \left.+z_{R}^{\prime 3}\left(\bar{q}_{(3)}+\frac{1}{2}\left\{\bar{q}_{(1)}, \bar{q}_{(2)}\right\}+\frac{1}{6} \bar{q}_{(1)}^{3}\right)+\mathcal{O}\left(z_{R}^{\prime 4}\right)\right] \mathrm{e}^{\bar{q}_{(0)} / 2}
\end{aligned}
$$

with the direct expansion using (4.33), $\bar{q}_{(n)}$ are obtained as

$$
\begin{aligned}
& \bar{q}_{(0)}=i \gamma^{-} T^{3} Q_{(0)}^{R, 3}, \\
& \bar{q}_{(1)}=\mathrm{e}^{-2 \beta} \sinh 2 \alpha\left(T^{+} Q_{(1)}^{R,-}+T^{-} \widetilde{Q}_{(1)}^{R,+}\right), \\
& \bar{q}_{(2)}=-\frac{i}{2} \mathrm{e}^{-4 \beta} \sinh ^{2} 2 \alpha T^{3} \bar{Q}_{(2)}^{R, 3},
\end{aligned}
$$




$$
\bar{q}_{(3)}=\frac{1}{4} \mathrm{e}^{-6 \beta} \sinh ^{3} 2 \alpha\left(T^{+} Q_{(3)}^{R,-}+T^{-} \widetilde{Q}_{(3)}^{R,+}\right) .
$$

Here the conserved quantities have been introduced as

$$
\begin{aligned}
& Q_{(0)}^{R, 3}=\int_{-\infty}^{\infty} d x j_{t}^{R, 3}(x) \quad\left(=Q^{R, 3}\right) \\
& Q_{(1)}^{R,-}=\int_{-\infty}^{\infty} d x j_{t}^{R,-}(x) \quad\left(=Q^{R,-}\right), \quad \widetilde{Q}_{(1)}^{R,+}=\int_{-\infty}^{\infty} d x \widetilde{j}_{t}^{R,+}(x) \quad\left(=\widetilde{Q}^{R,+}\right), \\
& \bar{Q}_{(2)}^{R, 3}=\int_{-\infty}^{\infty} d x \int_{-\infty}^{\infty} d y \epsilon(x-y) j_{t}^{R,-}(x) \widetilde{j}_{t}^{R,+}(y) \\
& -2 i \mathrm{e}^{2 \beta} \int_{-\infty}^{\infty} d x j_{x}^{R, 3}(x)+2 i \mathrm{e}^{2 \beta} \frac{\cosh 2 \alpha}{\sinh 2 \alpha} Q_{(0)}^{R, 3}, \\
& Q_{(3)}^{R,-}=\frac{1}{2} \int_{-\infty}^{\infty} d x \int_{-\infty}^{\infty} d y \int_{-\infty}^{\infty} d z \epsilon(x-y) \epsilon(x-z) \widetilde{j}_{t}^{R,+}(x) j_{t}^{R,-}(y) j_{t}^{R,-}(z) \\
& -2 i \mathrm{e}^{2 \beta} \int_{-\infty}^{\infty} d x \int_{-\infty}^{\infty} d y \epsilon(x-y) j_{t}^{R,-}(x)\left(j_{x}^{R, 3}(y)-\frac{\cosh 2 \alpha}{\sinh 2 \alpha} j_{t}^{R, 3}(y)\right) \\
& -\frac{4 \mathrm{e}^{2 \beta}}{\sinh 2 \alpha} \int_{-\infty}^{\infty} d x j_{x}^{R,-}(x)-\frac{1}{6} \widetilde{Q}_{(1)}^{R,+}\left(Q_{(1)}^{R,-}\right)^{2}+\frac{4 \mathrm{e}^{2 \beta} \cosh 2 \alpha}{\sinh ^{2} 2 \alpha} Q_{(1)}^{R,-}, \\
& \widetilde{Q}_{(3)}^{R,+}=\frac{1}{2} \int_{-\infty}^{\infty} d x \int_{-\infty}^{\infty} d y \int_{-\infty}^{\infty} d z \epsilon(x-y) \epsilon(x-z) j_{t}^{R,-}(x) \widetilde{j}_{t}^{R,+}(y) \widetilde{j}_{t}^{R,+}(z) \\
& +2 i \mathrm{e}^{2 \beta} \int_{-\infty}^{\infty} d x \int_{-\infty}^{\infty} d y \epsilon(x-y) \widetilde{j}_{t}^{R,+}(x)\left(j_{x}^{R, 3}(y)-\frac{\cosh 2 \alpha}{\sinh 2 \alpha} j_{t}^{R, 3}(y)\right) \\
& -\frac{4 \mathrm{e}^{2 \beta}}{\sinh 2 \alpha} \int_{-\infty}^{\infty} d x \widetilde{j}_{x}^{R,+}(x)-\frac{1}{6} Q_{(1)}^{R,+}\left(\widetilde{Q}_{(1)}^{R,+}\right)^{2}+\frac{4 \mathrm{e}^{2 \beta} \cosh 2 \alpha}{\sinh ^{2} 2 \alpha} \widetilde{Q}_{(1)}^{R,+} .
\end{aligned}
$$

These are also important to see the tower structure of the conserved charges in addition to the previous expansion.

\subsection{An infinite-dimensional extension of $q$-deformed $s u(2)_{\mathrm{R}}$}

The next is to compute the Poisson brackets of $Q_{(n)}^{R}$ and $\widetilde{Q}_{(n)}^{R}(n \geq 0)$.

For this purpose, the following Poisson brackets are useful,

$$
\begin{aligned}
& \left\{j_{t}^{R, 3}(x), j_{t}^{R, 3}(y)\right\}_{\mathrm{P}}=2 K \partial_{x} \delta(x-y), \\
& \left\{i_{t}^{R, \pm}(x), j_{t}^{R, 3}(y)\right\}_{\mathrm{P}}= \pm i i_{t}^{R, \pm}(x) \delta(x-y), \\
& \left\{i_{t}^{R, \pm}(x), i_{t}^{R, \mp}(y)\right\}_{\mathrm{P}}=\mp i j_{t}^{R, 3}(x) \delta(x-y)+2 K \partial_{x} \delta(x-y) . \\
& \left\{\widetilde{i}_{t}^{R, \pm}(x), j_{t}^{R, 3}(y)\right\}_{\mathrm{P}}= \pm i \tilde{i}_{t}^{R, \pm}(x) \delta(x-y),
\end{aligned}
$$




$$
\begin{aligned}
& \left\{\widetilde{i}_{t}^{R, \pm}(x), \widetilde{i}_{t}^{R, \mp}(y)\right\}_{\mathrm{P}}=\mp i j_{t}^{R, 3}(x) \delta(x-y)+2 K \partial_{x} \delta(x-y), \\
& \left\{i_{t}^{R, \pm}(x), \widetilde{i}_{t}^{R, \mp}(y)\right\}_{\mathrm{P}}=\mp i\left[\left(1 \mp \frac{2 \cosh ^{2} \alpha \sinh \beta}{\cosh \beta}\right) j_{t}^{R, 3}(x)\right. \\
& \left.\quad \pm 2 \sinh \alpha \cosh \alpha j_{x}^{R, 3}(x)\right] \delta(x-y) \\
& +2(K \pm i A) \partial_{x} \delta(x-y), \\
& \left\{j_{x}^{R, 3}(x), i_{t}^{R, \pm}(y)\right\}_{\mathrm{P}}=\mp i\left[\mathrm{e}^{ \pm 2 \beta} i_{t}^{R, \pm}(x) \pm \frac{\mathrm{e}^{ \pm \beta}(1-\cosh 2 \alpha \cosh 2 \beta)}{\sinh 2 \alpha \cosh \beta} i_{t}^{R, \pm}(x)\right] \delta(x-y), \\
& \left\{j_{x}^{R, 3}(x), \widetilde{i}_{t}^{R, \pm}(y)\right\}_{\mathrm{P}}=\mp i\left[\mathrm{e}^{\mp 2 \beta} \widetilde{i}_{t}^{R, \pm}(x) \mp \frac{\left.\mathrm{e}^{\mp \beta}(1-\cosh 2 \alpha \cosh 2 \beta) \widetilde{i n g}_{t}^{R, \pm}(x)\right] \delta(x-y) .}{\sinh 2 \alpha \cosh \beta}\right.
\end{aligned}
$$

Then, with the above relations, the Poisson brackets are computed as

$$
\begin{aligned}
& \left\{Q_{(1)}^{R, \pm}, Q_{(0)}^{R, 3}\right\}_{\mathrm{P}}= \pm i Q_{(1)}^{R, \pm} \\
& \left\{Q_{(1)}^{R,+}, Q_{(1)}^{R,-}\right\}_{\mathrm{P}}=-i \frac{\left(\gamma^{+}\right)^{2}+\left(\gamma^{-}\right)^{2}}{2 \gamma^{+} \gamma^{-}} \frac{2}{\left(\gamma^{+}+\gamma^{-}\right)} \sinh \left[\frac{\left(\gamma^{+}+\gamma^{-}\right)}{2} Q_{(0)}^{R, 3}\right], \\
& \left\{\widetilde{Q}_{(1)}^{R, \pm}, Q_{(0)}^{R, 3}\right\}_{\mathrm{P}}= \pm i Q_{(1)}^{R, \pm} \text {, } \\
& \left\{\widetilde{Q}_{(1)}^{R,+}, \widetilde{Q}_{(1)}^{R,-}\right\}_{\mathrm{P}}=-i \frac{\left(\gamma^{+}\right)^{2}+\left(\gamma^{-}\right)^{2}}{2 \gamma^{+} \gamma^{-}} \frac{2}{\left(\gamma^{+}+\gamma^{-}\right)} \sinh \left[\frac{\left(\gamma^{+}+\gamma^{-}\right)}{2} Q_{(0)}^{R, 3}\right], \\
& \left\{Q_{(1)}^{R,-}, \widetilde{Q}_{(1)}^{R,-}\right\}_{\mathrm{P}}=0, \\
& \left\{Q_{(1)}^{R,+}, \widetilde{Q}_{(1)}^{R,+}\right\}_{\mathrm{P}}=0, \\
& \left\{Q_{(1)}^{R,-}, \widetilde{Q}_{(1)}^{R,+}\right\}_{\mathrm{P}}=i \frac{\gamma^{-}}{\gamma^{+}} \gamma \bar{Q}_{(2)}^{R, 3}, \\
& \left\{Q_{(1)}^{R,+}, \widetilde{Q}_{(1)}^{R,-}\right\}_{\mathrm{P}}=i \frac{\gamma^{+}}{\gamma^{-}} \gamma Q_{(2)}^{R, 3}, \\
& \left\{\bar{Q}_{(2)}^{R, 3}, Q_{(1)}^{R,-}\right\}_{\mathrm{P}}=-i \frac{\gamma^{-}}{\gamma^{+}} \gamma\left[Q_{(3)}^{R,-}+\frac{2}{3} \widetilde{Q}_{(1)}^{R,+}\left(Q_{(1)}^{R,-}\right)^{2}\right], \\
& \left\{\bar{Q}_{(2)}^{R, 3}, \widetilde{Q}_{(1)}^{R,+}\right\}_{\mathrm{P}}=i \frac{\gamma^{-}}{\gamma^{+}} \gamma\left[\widetilde{Q}_{(3)}^{R,+}+\frac{2}{3} Q_{(1)}^{R,-}\left(\widetilde{Q}_{(1)}^{R,+}\right)^{2}\right] \text {, } \\
& \left\{Q_{(2)}^{R, 3}, \widetilde{Q}_{(1)}^{R,-}\right\}_{\mathrm{P}}=i \frac{\gamma^{+}}{\gamma^{-}} \gamma\left[\widetilde{Q}_{(3)}^{R,-}+\frac{2}{3} Q_{(1)}^{R,+}\left(\widetilde{Q}_{(1)}^{R,-}\right)^{2}\right], \\
& \left\{Q_{(2)}^{R, 3}, Q_{(1)}^{R,+}\right\}_{\mathrm{P}}=-i \frac{\gamma^{+}}{\gamma^{-}} \gamma\left[Q_{(3)}^{R,+}+\frac{2}{3} \widetilde{Q}_{(1)}^{R,-}\left(Q_{(1)}^{R,+}\right)^{2}\right] \text {, } \\
& \left\{Q_{(3)}^{R,-}, Q_{(1)}^{R,-}\right\}_{\mathrm{P}}=-\frac{i}{3} \frac{\gamma^{-}}{\gamma^{+}} \gamma\left(Q_{(1)}^{R,-}\right)^{2} \bar{Q}_{(2)}^{R, 3}, \\
& \left\{\widetilde{Q}_{(3)}^{R,+}, \widetilde{Q}_{(1)}^{R,+}\right\}_{\mathrm{P}}=\frac{i}{3} \frac{\gamma^{-}}{\gamma^{+}} \gamma\left(\widetilde{Q}_{(1)}^{R,+}\right)^{2} \bar{Q}_{(2)}^{R, 3}, \\
& \left\{\widetilde{Q}_{(3)}^{R,-}, \widetilde{Q}_{(1)}^{R,-}\right\}_{\mathrm{P}}=\frac{i}{3} \frac{\gamma^{+}}{\gamma^{-}} \gamma\left(\widetilde{Q}_{(1)}^{R,-}\right)^{2} Q_{(2)}^{R, 3},
\end{aligned}
$$




$$
\left\{Q_{(3)}^{R,+}, Q_{(1)}^{R,+}\right\}_{\mathrm{P}}=-\frac{i}{3} \frac{\gamma^{+}}{\gamma^{-}} \gamma\left(Q_{(1)}^{R,+}\right)^{2} Q_{(2)}^{R, 3}
$$

Again, we should be careful for non-ultra local terms. For the detail, see Appendix B.

In addition, the Serre-like relations are evaluated as follows:

$$
\begin{aligned}
& \left\{Q_{(1)}^{R, \pm},\left\{Q_{(1)}^{R, \pm},\left\{Q_{(1)}^{R, \pm}, \widetilde{Q}_{(1)}^{R, \mp}\right\}_{\mathrm{P}}\right\}_{\mathrm{P}}\right\}_{\mathrm{P}}=-\left(\frac{\gamma^{ \pm}}{\gamma^{\mp}} \gamma\right)^{2}\left(Q_{(1)}^{R, \pm}\right)^{2}\left\{Q_{(1)}^{R, \pm}, \widetilde{Q}_{(1)}^{R, \mp}\right\}_{\mathrm{P}}, \\
& \left\{\widetilde{Q}_{(1)}^{R, \pm},\left\{\widetilde{Q}_{(1)}^{R, \pm},\left\{\widetilde{Q}_{(1)}^{R, \pm}, Q_{(1)}^{R, \mp}\right\}_{\mathrm{P}}\right\}_{\mathrm{P}}\right\}_{\mathrm{P}}=-\left(\frac{\gamma^{\mp}}{\gamma^{ \pm}} \gamma\right)^{2}\left(\widetilde{Q}_{(1)}^{R, \pm}\right)^{2}\left\{\widetilde{Q}_{(1)}^{R, \pm}, Q_{(1)}^{R, \mp}\right\}_{\mathrm{P}} .
\end{aligned}
$$

The relations in (4.37) may be interpreted as deformations of the classical analogue of $q$-Serre

relations in the standard quantum affine algebra $U_{q}(\widehat{s u(2)})$ and ensure that the resulting algebra exhibits the similar tower structure. The higher-level charges are obtained from the level 1 charges by taking the Poisson bracket repeatedly as with $\left.U_{q}(\widehat{s u(2)})_{\mathrm{R}}\right)$. The deformed algebra obtained here may be lifted up to the quantum level. Although we have not yet succeeded to find out its mathematical formulation, a two-parameter quantum toroidal algebra [34] would be a possible candidate of it.

The tower structure of the conserved charges is depicted in Fig.2, The overall coefficient of the Poisson bracket is affected by the deformation. When going up the tower of charges, the factor $\gamma^{-} / \gamma^{+}$is multiplied to the Poisson bracket. On the other hand, when going down the tower, the factor $\gamma^{+} / \gamma^{-}$is multiplied to the Poisson bracket.

When $\gamma^{+} / \gamma^{-}=\mathrm{e}^{2 \beta}= \pm 1$, the relations in (4.37) are reduced to the classical analogue of $q$-Serre relations. The first condition $\mathrm{e}^{2 \beta}=1$ means that $K=0$ and corresponds to squashed sigma models without the Wess-Zumino term. The second condition $\mathrm{e}^{2 \beta}=-1$ means $K=0$ and $C=-1$. In the $C \rightarrow-1$ limit, $O(3)$ non-linear sigma models are reproduced as it is obvious from the classical action. However, because this limit is singular, the second condition would also be singular.

\subsection{The $r / s$-matrices}

The $r / s$-matrices associated with the right Lax pair in (4.1) are computed from the Poisson bracket between the spatial components of the Lax pair,

$$
\begin{aligned}
\left\{L_{x}^{R}\left(x ; \lambda_{R}\right) \stackrel{\otimes}{,} L_{x}^{R}\left(y ; \mu_{R}\right)\right\}_{\mathrm{P}}= & {\left[r^{R}\left(\lambda_{R}, \mu_{R}\right), L_{x}^{R}\left(x ; \lambda_{R}\right) \otimes 1+1 \otimes L_{x}^{R}\left(y ; \mu_{R}\right)\right] \delta(x-y) } \\
& -\left[s^{R}\left(\lambda_{R}, \mu_{R}\right), L_{x}^{R}\left(x ; \lambda_{R}\right) \otimes 1-1 \otimes L_{x}^{R}\left(y ; \mu_{R}\right)\right] \delta(x-y)
\end{aligned}
$$




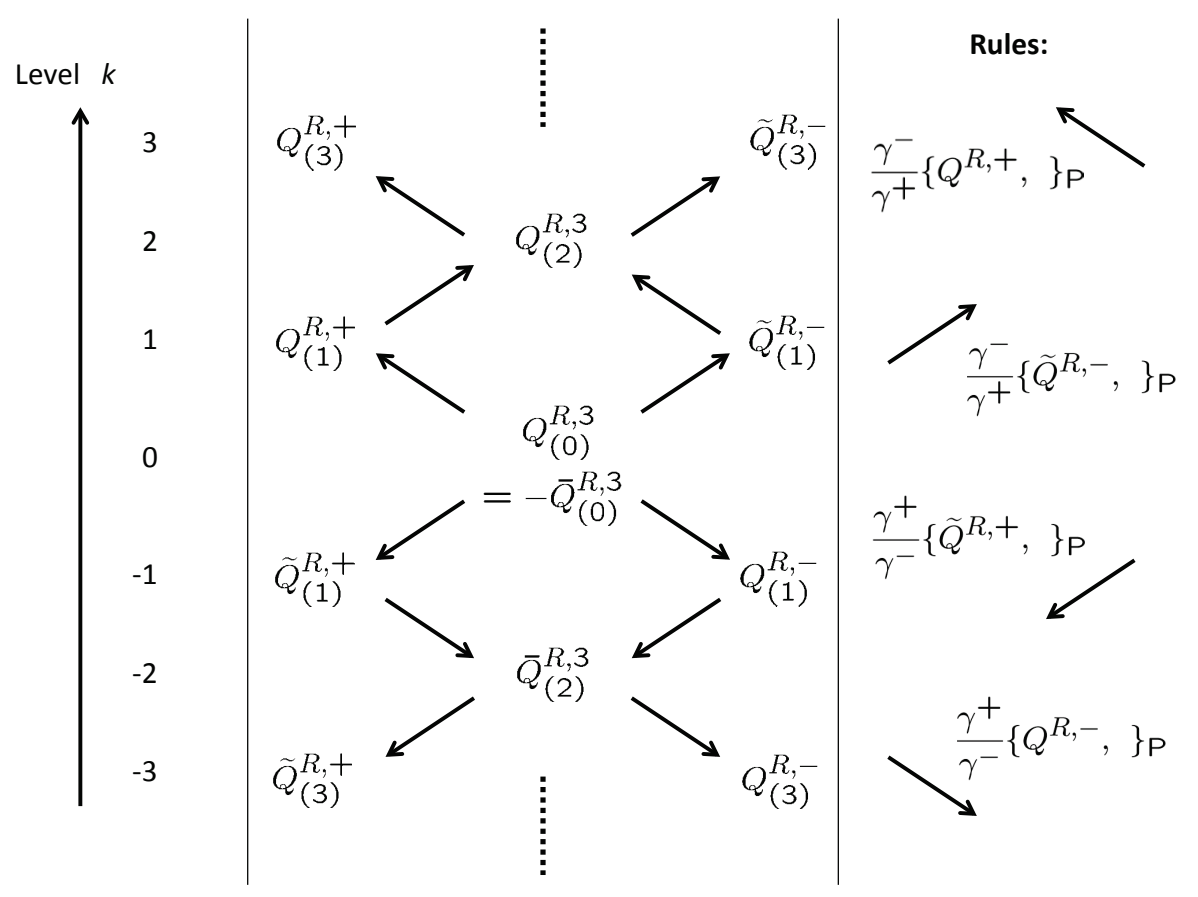

Figure 2: The tower structure of the conserved charges.

$$
-2 s^{R}\left(\lambda_{R}, \mu_{R}\right) \partial_{x} \delta(x-y)
$$

The resulting $r / s$-matrices are

$$
\begin{aligned}
r^{R}\left(\lambda_{R}, \mu_{R}\right)= & \frac{h_{\alpha, \beta}^{R}\left(\lambda_{R}\right)+h_{\alpha, \beta}^{R}\left(\mu_{R}\right)}{2 \sinh \left(\lambda_{R}-\mu_{R}\right)}\left[T^{-} \otimes T^{+}+T^{+} \otimes T^{-}+\cosh \left(\lambda_{R}-\mu_{R}\right) T^{3} \otimes T^{3}\right] \\
& -\frac{h_{\alpha, \beta}^{R}\left(\lambda_{R}\right)-h_{\alpha, \beta}^{R}\left(\mu_{R}\right)}{2} \tanh \beta T^{3} \otimes T^{3}, \\
s^{R}\left(\lambda_{R}, \mu_{R}\right)= & \frac{h_{\alpha, \beta}^{R}\left(\lambda_{R}\right)-h_{\alpha, \beta}^{R}\left(\mu_{R}\right)}{2 \sinh \left(\lambda_{R}-\mu_{R}\right)}\left[T^{-} \otimes T^{+}+T^{+} \otimes T^{-}+\cosh \left(\lambda_{R}-\mu_{R}\right) T^{3} \otimes T^{3}\right] \\
& -\frac{h_{\alpha, \beta}^{R}\left(\lambda_{R}\right)+h_{\alpha, \beta}^{R}\left(\mu_{R}\right)}{2} \tanh \beta T^{3} \otimes T^{3},
\end{aligned}
$$

where a scalar function $h_{\alpha, \beta}^{R}(\lambda)$ is defined as

$$
h_{\alpha, \beta}^{R}(\lambda) \equiv \frac{\sinh 2 \alpha \sinh ^{2} \lambda}{2 \sinh (\alpha+\beta+\lambda) \sinh (\alpha-\beta-\lambda)} .
$$

It is straightforward to show that the classical $r / s$-matrices given in (4.38) satisfy the extended classical Yang-Baxter equation,

$$
\left[(r-s)_{12}^{R}\left(\lambda_{R}, \mu_{R}\right),(r+s)_{13}^{R}\left(\lambda_{R}, \nu_{R}\right)\right]+\left[(r+s)_{12}^{R}\left(\lambda_{R}, \mu_{R}\right),(r+s)_{23}^{R}\left(\mu_{R}, \nu_{R}\right)\right]
$$




$$
+\left[(r+s)_{13}^{R}\left(\lambda_{R}, \nu_{R}\right),(r+s)_{23}^{R}\left(\mu_{R}, \nu_{R}\right)\right]=0
$$

Thus the classical integrability has been ensured $\ddagger$.

In the previous subsection, a deformation of the quantum affine algebra has been shown explicitly. According to the deformation, the $r / s$-matrices given in (4.38) are also deformed by additional terms proportional to $\tanh \beta T^{3} \otimes T^{3}$.

It seems likely that the additional terms cannot be eliminated by any gauge transformations. This would indicate that the deformed quantum affine algebra cannot be mapped to the standard quantum affine algebra.

When $\beta=0$ and $\alpha \neq 0$ (i.e., $K=0), h_{\alpha, \beta}^{R}(\lambda)$ is reduced to

$$
h_{\alpha, 0}^{R}(\lambda) \equiv \frac{\sinh 2 \alpha \sinh ^{2} \lambda}{2 \sinh (\alpha+\lambda) \sinh (\alpha-\lambda)},
$$

and the deformation terms proportional to $\tanh \beta T^{3} \otimes T^{3}$ vanish. Thus the $r / s$-matrices obtained in [26] are reproduced.

\section{The left-right duality}

Let us show the gauge-equivalence, which is referred to as left-right duality, between the right Lax pair $L_{\mu}^{R}\left(\lambda_{R}\right)$ and a pair of the left Lax pairs $L_{\mu}^{L_{ \pm}}\left(\lambda_{L_{ \pm}}\right)$under a certain relation between the spectral parameters and the rescaling of $s l(2)$ generators. The equivalence is shown in the case without the Wess-Zumino term [27]. The analysis here is a generalization of the result obtained in [27].

\section{The fundamental domains of the spectral parameters}

It is useful to realize the fundamental domains of the spectral parameters.

Recall that a pair of the Lax pairs in the left description [See (3.5)] are given by

$$
L_{t}^{L_{ \pm}}\left(x ; \lambda_{L_{ \pm}}\right)=\frac{1}{2}\left[L_{+}^{L_{ \pm}}\left(x ; \lambda_{L_{ \pm}}\right)+L_{-}^{L_{ \pm}}\left(x ; \lambda_{L_{ \pm}}\right)\right]
$$

\footnotetext{
$\ddagger$ The $r / s$-matrices depend on $\lambda_{R}$ and $\mu_{R}$ individually (not only $\lambda_{R}-\mu_{R}$ ), though they satisfy the extended classical Yang-Baxter equation. Hence it is unclear to classify the $r / s$-matrices in a well-known manner.
} 

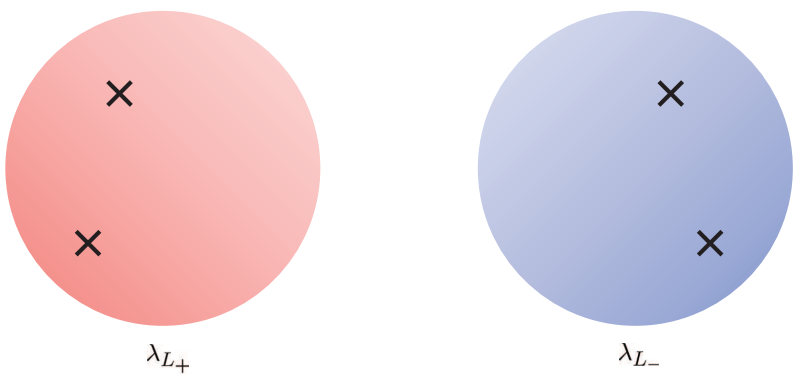

Figure 3: Two Riemann spheres with two punctures.

$$
\begin{aligned}
& L_{x}^{L_{ \pm}}\left(x ; \lambda_{L_{ \pm}}\right)=\frac{1}{2}\left[L_{+}^{L_{ \pm}}\left(x ; \lambda_{L_{ \pm}}\right)-L_{-}^{L_{ \pm}}\left(x ; \lambda_{L_{ \pm}}\right)\right] \\
& L_{+}^{L_{ \pm}}\left(x ; \lambda_{L_{ \pm}}\right)=\frac{1}{1+\lambda_{L_{ \pm}}} j_{+}^{L_{ \pm}}, \quad L_{-}^{L_{ \pm}}\left(x ; \lambda_{L_{ \pm}}\right)=\frac{1}{1-\lambda_{L_{ \pm}}} j_{-}^{L_{ \pm}},
\end{aligned}
$$

where each of the spectral parameters take the values on a Riemann sphere with two punctures: $\lambda_{L_{ \pm}} \in \mathbb{C} \cup\{\infty\}$. The punctures come from the fact that each Lax pair has two poles at $\lambda_{L_{ \pm}}= \pm 1$, as depicted in Fig. 3 .

On the other hand, the Lax pair in the right description [See (4.1)] is given by

$$
\begin{aligned}
& L_{t}^{R}\left(x ; \lambda_{R}\right)=\frac{1}{2}\left[L_{+}^{R}\left(x ; \lambda_{R}\right)+L_{-}^{R}\left(x ; \lambda_{R}\right)\right], \\
& L_{x}^{R}\left(x ; \lambda_{R}\right)=\frac{1}{2}\left[L_{+}^{R}\left(x ; \lambda_{R}\right)-L_{-}^{R}\left(x ; \lambda_{R}\right)\right], \\
& L_{ \pm}^{R}\left(x ; \lambda_{R}\right)=-\frac{\sinh (\alpha \pm \beta)}{\sinh \left[\alpha \pm\left(\beta+\lambda_{R}\right)\right]}\left[T^{+} J_{ \pm}^{-}+T^{-} J_{ \pm}^{+}+\frac{\cosh \left(\alpha \pm \lambda_{R}\right)}{\cosh \alpha} T^{3} J_{ \pm}^{3}\right] .
\end{aligned}
$$

The spectral parameter $\lambda_{R}$ is periodically identified as

$$
\lambda_{R} \sim \lambda_{R}+2 \pi i
$$

and hence it takes the values on a cylinder. Because the right Lax pair is regular in the $\operatorname{Re} \lambda_{R} \rightarrow \pm \infty$ limit, the fundamental domain of spectral parameter can be regarded as a Riemann sphere under the map

$$
\lambda_{R} \rightarrow z_{R}=\mathrm{e}^{\lambda_{R}} .
$$

It is obvious that the right Lax pair has four poles at

$$
z_{R}=\mathrm{e}^{ \pm \alpha-\beta}, \quad-\mathrm{e}^{ \pm \alpha-\beta} .
$$




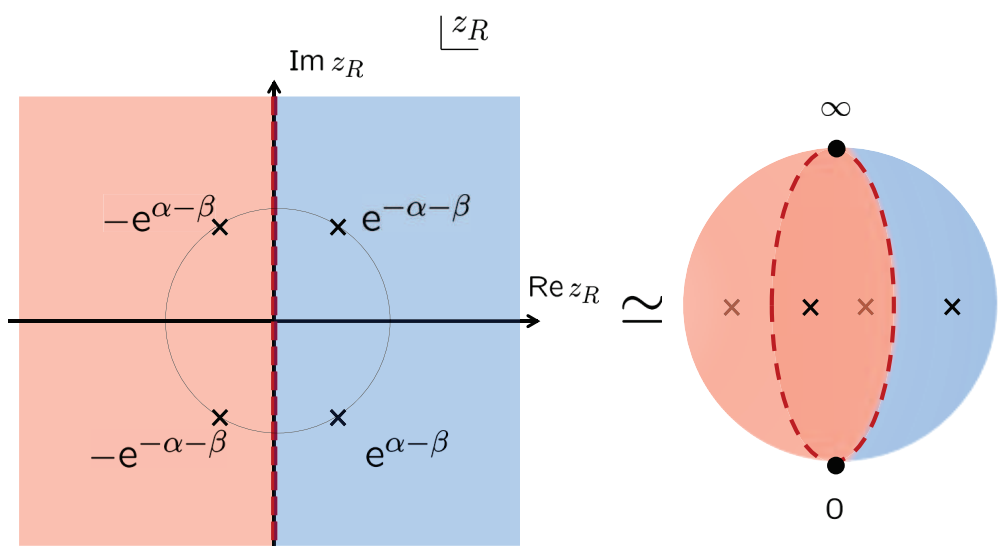

Figure 4: The Riemann sphere with four punctures for $\alpha$ and $\beta$ are purely imaginary.

Thus the domain of the spectral parameter $z_{R}$ is regarded as a Riemann sphere with four punctures as depicted in Fig. 䢞.

In the following, we show that the Riemann sphere of $z_{R}$ is related to a pair of the Riemann spheres of $\lambda_{L_{ \pm}}$.

\section{The reduced right descriptions}

As a next step, we introduce the reduced right descriptions. Lax pairs in the reduced right descriptions are obtained from the original right Lax pair through the following isomorphisms of the $\operatorname{sl}(2)$ algebra,

$$
\begin{aligned}
T^{ \pm} & \rightarrow \mathrm{e}^{ \pm \lambda_{R}} T^{ \pm}=\mathrm{e}^{-i \lambda_{R} T^{3}} T^{ \pm} \mathrm{e}^{i \lambda_{R} T^{3}} \\
\text { or } \quad T^{ \pm} & \rightarrow \mathrm{e}^{\mp \lambda_{R}} T^{ \pm}=\mathrm{e}^{i \lambda_{R} T^{3}} T^{ \pm} \mathrm{e}^{-i \lambda_{R} T^{3}} .
\end{aligned}
$$

Because $T^{3}$ is invariant under the isomorphisms, the generators can be rewritten into

$$
T^{a} \rightarrow \mathrm{e}^{\mp i \lambda_{R} T^{3}} T^{a} \mathrm{e}^{ \pm i \lambda_{R} T^{3}}
$$

One of the resulting Lax pairs is

$$
L_{ \pm}^{R_{+}}\left(x ; \lambda_{R_{+}}\right)=-\frac{\sinh (\alpha \pm \beta)}{\sinh \left[\alpha \pm\left(\beta+\lambda_{R_{+}}\right)\right]}
$$

$\S$ When $\alpha$ and $\beta$ are real, the four poles are on the real axis of the $z_{R}$-plane. When $\alpha$ and $\beta$ are purely imaginary, the four poles are on the unit circle with the center at the origin. 


$$
\times\left[\mathrm{e}^{+\lambda_{R_{+}}} T^{+} J_{ \pm}^{-}+\mathrm{e}^{-\lambda_{R_{+}}} T^{-} J_{ \pm}^{+}+\frac{\cosh \left(\alpha \pm \lambda_{R_{+}}\right)}{\cosh \alpha} T^{3} J_{ \pm}^{3}\right] .
$$

For later convenience, we change the subscript of the spectral parameter from $R$ to $R_{+}$. This reduced right Lax pair corresponds to the former isomorphism (5.4). Similarly, the other reduced right Lax pair is defined as

$$
\begin{aligned}
& L_{ \pm}^{R_{-}}\left(x ; \lambda_{R_{-}}\right)=-\frac{\sinh (\alpha \pm \beta)}{\sinh \left[\alpha \pm\left(\beta+\lambda_{R_{-}}\right)\right]} \\
& \times\left[\mathrm{e}^{-\lambda_{R_{-}}} T^{+} J_{ \pm}^{-}+\mathrm{e}^{+\lambda_{R_{-}}} T^{-} J_{ \pm}^{+}+\frac{\cosh \left(\alpha \pm \lambda_{R_{-}}\right)}{\cosh \alpha} T^{3} J_{ \pm}^{3}\right] .
\end{aligned}
$$

For this reduced right Lax pair, its spectral parameter is denoted as $\lambda_{R_{-}}$. This reduced right Lax pair corresponds to the later isomorphism (5.5) .

Note that the domains of the spectral parameters $\lambda_{R_{ \pm}}$have the periodicities,

$$
\lambda_{R_{ \pm}} \sim \lambda_{R_{ \pm}}+\pi i
$$

and hence the squares of $z_{R_{ \pm}}$

$$
z_{R_{ \pm}}^{2}=\mathrm{e}^{2 \lambda_{R_{ \pm}}}
$$

live on the Riemann spheres Each of the reduced right Lax pairs $L_{\mu}^{R_{ \pm}}\left(\lambda_{R_{ \pm}}\right)$has two poles in its fundamental domain,

$$
z_{R_{ \pm}}^{2}=\mathrm{e}^{2 \alpha-2 \beta} \quad \text { and } \quad \mathrm{e}^{-2 \alpha-2 \beta} .
$$

As a result, the fundamental domains of $z_{R_{ \pm}}^{2}$ are a pair of Riemann spheres with two punctures.

So far, we have shown that the right description is decomposed to a couple of the reduced right descriptions. The statement we want to show is that each of the Lax pair in the reduced right description is equivalent to each of the Lax pairs in the left description through a gauge transformation with a certain identification of the spectral parameters.

\section{A relation of the spectral parameters}

Then the next task is to find out a relation of the spectral parameters in the left and right descriptions. Because both $\lambda_{L_{ \pm}}$and $z_{R_{ \pm}}^{2}$ live on the two-punctured Riemann spheres, they

\footnotetext{
I Note that $z_{R_{ \pm}}$and $-z_{R_{ \pm}}$cannot be distinguished and hence $z_{R_{ \pm}}^{2}$ takes the value on a Riemann sphere.
} 
should be related through a Möbius transformation,

$$
z_{R_{ \pm}}^{2}=\frac{a \lambda_{L_{ \pm}}+b}{c \lambda_{L_{ \pm}}+d}
$$

The constant parameter should be fixed by the pole structure and the correspondence of the expansion points for Yangians.

The first requirement is that the position of the poles should be mapped each other. This condition leads to

$$
\mathrm{e}^{ \pm 2 \alpha-2 \beta}=\frac{a \pm b}{c \pm d}
$$

Now we have two possibilities: the pole at $z_{R_{ \pm}}^{2}=\mathrm{e}^{2 \alpha-2 \beta}$ corresponds to 1) the pole at $\lambda_{L_{ \pm}}=1$, or 2) the pole at $\lambda_{L_{ \pm}}=-1$. However, we should here take the possibility 1) so that the resulting Möbius transformation reproduces the result in [27] when $K=0$.

Then, recall that the Yangian charges $Q_{(n)}^{L_{ \pm}}$are obtained by expanding the (reduced) right Lax pairs around $z_{R_{ \pm}}^{2}=1$. From this information, the following relation is obtained,

$$
1=\frac{a}{c}
$$

Thus the final result is given by

$$
z_{R_{ \pm}}^{2}=\frac{\sinh 2 \alpha \lambda_{L_{ \pm}}+\cosh 2 \alpha-\mathrm{e}^{-2 \beta}}{\sinh 2 \alpha \lambda_{L_{ \pm}}-\cosh 2 \alpha+\mathrm{e}^{2 \beta}}=\frac{\lambda_{L_{ \pm}}+K+i A}{\lambda_{L_{ \pm}}+K-i A},
$$

or equivalently,

$$
\frac{\lambda_{L_{ \pm}}+K}{i A}=\frac{1}{\tanh \lambda_{R_{ \pm}}} .
$$

Here we comment on the connection between the two-punctured $\lambda_{L}$-Riemann spheres and the four-punctured $z_{R}$-Riemann sphere. The relation between $z_{R}$ and $\lambda_{L}$ is also given by $(5.15)$ :

$$
z_{R}^{2}=\frac{\lambda_{L_{ \pm}}+K+i A}{\lambda_{L_{ \pm}}+K-i A} .
$$

However the interpretation is more involved because $-z_{R}$ is certainly a different from the point $z_{R}$. As a relation between $z_{R}$ and $\lambda_{L_{ \pm}}$(not $z_{R}^{2}$ and $\lambda_{L_{ \pm}}$), the relation in (5.17) indicates that there exists a cut between $\lambda_{L_{ \pm}}=-K+i A$ and $\lambda_{L_{ \pm}}=-K-i A$. Two-punctured $\lambda_{L^{-}}$ Riemann spheres are connected along the cut and combined into a single Riemann sphere with four punctures. The resulting four-punctured Riemann sphere is depicted in Fig 5 . 


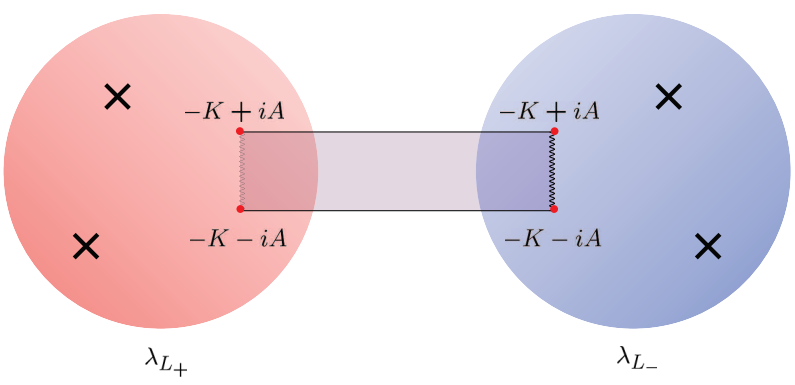

Figure 5: Two-punctured $\lambda_{L}$-Riemann spheres are connected along the cut.

\section{The gauge equivalence}

We concentrate on showing the gauge equivalence between $L_{\mu}^{R_{+}}\left(x ; \lambda_{R_{+}}\right)$and $L_{\mu}^{L_{+}}\left(x ; \lambda_{L_{+}}\right)$. The analysis for the case with (-)-subscript is also similar. The gauge transformation is generated by $g$ and the relation between the spectral parameters is given by

$$
\frac{\lambda_{L_{+}}+K}{i A}=\frac{1}{\tanh \lambda_{R_{+}}} .
$$

First of all, the left Lax pair $L_{\mu}^{L_{+}}\left(x ; \lambda_{L_{+}}\right)$can be rewritten as

$$
\begin{aligned}
& L_{ \pm}^{L_{+}}\left(x ; \lambda_{L_{+}}\right) \\
= & \frac{1}{1 \pm \lambda_{L_{+}}} g\left(J_{ \pm}-2 C \operatorname{tr}\left(T^{3} J_{ \pm}\right) T^{3} \mp K J_{ \pm} \mp A\left[J_{ \pm}, T^{3}\right]\right) g^{-1} \\
= & \frac{1}{1 \pm \lambda_{L_{+}}} g\left[T^{+}(1 \mp K \mp i A) J_{ \pm}^{-}+T^{-}(1 \mp K \pm i A) J_{ \pm}^{+}\right. \\
& \left.+T^{3}(1+C \mp K) J_{ \pm}^{3}\right] g^{-1} .
\end{aligned}
$$

Then a gauge-transformation of the left Lax pair is given by

$$
\begin{aligned}
& {\left[L_{ \pm}^{L_{+}}\left(x ; \lambda_{L_{+}}\right)\right]^{g} } \\
\equiv & g^{-1} L_{ \pm}^{L_{+}}\left(x ; \lambda_{L_{+}}\right) g-g^{-1} \partial_{ \pm} g \\
= & -J_{ \pm}+\frac{1}{1 \pm \lambda_{L_{+}}}\left[T^{+}(1 \mp K \mp i A) J_{ \pm}^{-}+T^{-}(1 \mp K \pm i A) J_{ \pm}^{+}\right. \\
& \left.\quad+T^{3}(1+C \mp K) J_{ \pm}^{3}\right] \\
= & -\left[\frac{\lambda_{L_{+}}+K+i A}{\lambda_{L_{+}} \pm 1} T^{+} J_{ \pm}^{-}+\frac{\lambda_{L_{+}}+K-i A}{\lambda_{L_{+}} \pm 1} T^{-} J_{ \pm}^{+}+\frac{\lambda_{L_{+}}+K \mp C}{\lambda_{L_{+}} \pm 1} T^{3} J_{ \pm}^{3}\right] .
\end{aligned}
$$


Finally, with the relation between the spectral parameters (5.18), it becomes the reduced right Lax pair:

$$
\begin{aligned}
& {\left[L_{ \pm}^{L_{+}}\left(x ; \lambda_{L_{+}}\right)\right]^{g} } \\
= & -\frac{\sinh (\alpha \pm \beta)}{\sinh \left[\alpha \pm\left(\beta+\lambda_{R_{+}}\right)\right]}\left[\mathrm{e}^{+\lambda_{R_{+}}} T^{+} J_{ \pm}^{-}+\mathrm{e}^{-\lambda_{R_{-}}} T^{-} J_{ \pm}^{+}+\frac{\cosh \left(\alpha \pm \lambda_{R_{+}}\right)}{\cosh \alpha} T^{3} J_{ \pm}^{3}\right] \\
= & L_{ \pm}^{R_{+}}\left(x ; \lambda_{R_{+}}\right) .
\end{aligned}
$$

Similarly, with the spectral-parameter relation

$$
\frac{\lambda_{L_{-}}+K}{i A}=\frac{1}{\tanh \lambda_{R_{-}}}
$$

we can show the gauge equivalence between the other left Lax pair $L_{\mu}^{L_{-}}\left(x ; \lambda_{L_{-}}\right)$and the other reduced right Lax pair $L_{\mu}^{R}\left(x ; \lambda_{R_{-}}\right)$:

$$
\left[L_{ \pm}^{L_{-}}\left(x ; \lambda_{L_{-}}\right)\right]^{g}=L_{ \pm}^{R_{-}}\left(x ; \lambda_{R_{-}}\right) .
$$

\section{Summary}

Let us summarize the results obtained so far. The two reduced right Lax pairs $L_{\mu}^{R_{ \pm}}\left(x ; \lambda_{R_{ \pm}}\right)$ have been introduced. Then they are obtained from the right Lax pair through the $\operatorname{sl}(2)$ isomorphisms,

$$
T^{a} \rightarrow \mathrm{e}^{\mp i \lambda_{R} T^{3}} T^{a} \mathrm{e}^{ \pm i \lambda_{R} T^{3}}
$$

The reduced right Lax pairs $L_{\mu}^{R_{ \pm}}\left(x ; \lambda_{R_{ \pm}}\right)$are related to the left Lax pairs $L_{\mu}^{L_{ \pm}}\left(x ; \lambda_{L_{ \pm}}\right)$through the gauge transformation generated by $g$,

$$
\left[L_{\mu}^{L_{ \pm}}\left(x ; \lambda_{L_{ \pm}}\right)\right]^{g}=L_{\mu}^{R_{ \pm}}\left(x ; \lambda_{R_{ \pm}}\right),
$$

under the relations between the spectral parameters

$$
\frac{\lambda_{L_{ \pm}}+K}{i A}=\frac{1}{\tanh \lambda_{R_{ \pm}}} .
$$

So far we have shown that $U^{L_{ \pm}}\left(\lambda_{L_{ \pm}}\right)$are gauge-equivalent to $U^{R_{ \pm}}\left(\lambda_{R_{ \pm}}\right)$and hence the charges for the deformed $\left.U_{q}(\widehat{s u(2)})_{\mathrm{R}}\right)$ can also be obtained from $U^{L_{ \pm}}\left(\lambda_{L_{ \pm}}\right)$. One can read off the expansion points from the relation (5.26) and the fact that the expansion points for $U^{R_{ \pm}}\left(\lambda_{R_{ \pm}}\right)$are $z_{R_{ \pm}}=0$ and $z_{R_{ \pm}}=\infty$. Thus the charges for the deformed $\left.\widehat{U_{q}(\widehat{s u(2)}} \widehat{\mathrm{R}}\right)$ are obtained by expanding $U^{L_{ \pm}}\left(\lambda_{L_{ \pm}}\right)$with respect to $\lambda_{L_{ \pm}}$around $\lambda_{L_{ \pm}}=-K-i A$ and $\lambda_{L_{ \pm}}=-K+i A$. The locations of poles and the expansion points for the deformed $\left.U_{q}(\widehat{s u(2)})_{\mathrm{R}}\right)$ and $\mathcal{Y}\left(s u(2)_{\mathrm{L}}\right)$ are summarized in Tab.1. 


\begin{tabular}{c||c|c}
\hline Charges $\backslash$ Monodromies & $U^{R_{ \pm}}\left(\lambda_{R_{ \pm}}\right)$ & $U^{L_{ \pm}}\left(\lambda_{L_{ \pm}}\right)$ \\
\hline \hline$Q_{(0)}^{R, 3}, Q_{(1)}^{R,+}, \widetilde{Q}_{(1)}^{R,-}$ & 0 & $-K-i A$ \\
\hline$Q_{(0)}^{R, 3}, Q_{(1)}^{R,-}, \widetilde{Q}_{(1)}^{R,+}$ & $\infty$ & $-K+i A$ \\
\hline$Q_{(0)}^{L, a}, Q_{(1)}^{L, a}$ & 1 & $\infty$ \\
\hline \hline local charges & $\mathrm{e}^{ \pm 2 \alpha-2 \beta}$ & \pm 1 \\
\hline
\end{tabular}

Table 1: The conserved charges and the expansion points of monodromy matrices are listed. The expansion points of $U^{R_{ \pm}}\left(\lambda_{R_{ \pm}}\right)$are described in terms of $z_{R_{ \pm}}^{2}$.

\section{The gauge transformation of the $r / s$-matrices}

The $r / s$-matrices for the reduced right Lax pairs can be obtained from the $r / s$-matrices for the left Lax pairs. Recall the gauge-transformation laws of the $r / s$-matrices,

$$
\begin{aligned}
& (r+s)^{R_{ \pm}}\left(\lambda_{R_{ \pm}}, \mu_{R_{ \pm}}\right) \delta(x-y) \\
= & g^{-1}(x) \otimes g^{-1}(y) \\
& \times\left[(r+s)^{L_{ \pm}}\left(\lambda_{L_{ \pm}}, \mu_{L_{ \pm}}\right) \delta(x-y)-\left\{L_{x}^{L_{ \pm}}\left(x ; \lambda_{L_{ \pm}}\right) \stackrel{\otimes}{,} g(y)\right\}_{\mathrm{P}} 1 \otimes g^{-1}(y)\right] \\
& \times g(x) \otimes g(y), \\
& (r-s)^{R_{ \pm}}\left(\lambda_{R_{ \pm}}, \mu_{R_{ \pm}}\right) \delta(x-y) \\
= & g^{-1}(x) \otimes g^{-1}(y) \\
& \times\left[(r-s)^{L_{ \pm}}\left(\lambda_{L_{ \pm}}, \mu_{L_{ \pm}}\right) \delta(x-y)-\left\{g(x) \stackrel{\otimes}{,} L_{x}^{L_{ \pm}}\left(y ; \mu_{L_{ \pm}}\right)\right\}_{\mathrm{P}} 1 \otimes g^{-1}(y)\right] \\
& \times g(x) \otimes g(y) .
\end{aligned}
$$

Thus the Poisson brackets

$$
\begin{aligned}
& \left\{L_{x}^{L_{ \pm}}\left(x ; \lambda_{L}\right) \otimes, g(y)\right\}_{\mathrm{P}} 1 \otimes g^{-1}(y) \\
& =\frac{-1}{1-\lambda_{L}^{2}}\left\{\left(-\lambda_{L}-\frac{K}{1+C}\right)\left(T^{-} \otimes T^{+}+T^{+} \otimes T^{-}+T^{3} \otimes T^{3}\right)\right. \\
& \quad \quad \pm A\left[T^{-} \otimes T^{+}+T^{+} \otimes T^{-}+T^{3} \otimes T^{3}, 1 \otimes g T^{3} g^{-1}(y)\right] \\
& \left.\quad \quad+\frac{C K}{1+C}\left[\left[T^{-} \otimes T^{+}+T^{+} \otimes T^{-}+T^{3} \otimes T^{3}, 1 \otimes g T^{3} g^{-1}(y)\right], 1 \otimes g T^{3} g^{-1}(y)\right]\right\} \\
& \quad \times \delta(x-y), \\
& \left\{g(x) \otimes, L_{x}^{L_{ \pm}}\left(y ; \mu_{L}\right)\right\}_{\mathrm{P}} g^{-1}(x) \otimes 1
\end{aligned}
$$




$$
\begin{aligned}
=\frac{1}{1-\mu_{L}^{2}}\{ & \left(-\mu_{L}-\frac{K}{1+C}\right)\left(T^{-} \otimes T^{+}+T^{+} \otimes T^{-}+T^{3} \otimes T^{3}\right) \\
& \pm A\left[T^{-} \otimes T^{+}+T^{+} \otimes T^{-}+T^{3} \otimes T^{3}, g T^{3} g^{-1}(x) \otimes 1\right] \\
& \left.\quad+\frac{C K}{1+C}\left[\left[T^{-} \otimes T^{+}+T^{+} \otimes T^{-}+T^{3} \otimes T^{3}, g T^{3} g^{-1}(x) \otimes 1\right], g T^{3} g^{-1}(x) \otimes 1\right]\right\} \\
\quad \times & \delta(x-y),
\end{aligned}
$$

are needed to compute the $r / s$-matrices for the reduced right Lax pair.

With the explicit expression of the $r / s$-matrices for the left Lax pairs (3.13), the relation (5.29) and the maps between spectral parameters (5.26), we can evaluate the right hand side of (5.27) as

$$
\begin{aligned}
& (r+s)^{R_{ \pm}}\left(\lambda_{R_{ \pm}}, \mu_{R_{ \pm}}\right) \delta(x-y) \\
= & \frac{1}{\sinh \left(\lambda_{R_{ \pm}}-\mu_{R_{ \pm}}\right)} \frac{\sinh 2 \alpha \sinh ^{2} \lambda_{R_{ \pm}}}{2 \sinh \left(\alpha+\beta+\lambda_{R_{ \pm}}\right) \sinh \left(\alpha-\beta-\lambda_{R_{ \pm}}\right)} \\
& \times\left\{\left[\cosh \left(\lambda_{R_{ \pm}}-\mu_{R_{ \pm}}\right)-\tanh \beta \sinh \left(\lambda_{R_{ \pm}}-\mu_{R_{ \pm}}\right)\right] T^{3} \otimes T^{3}\right. \\
& \left.\quad+\mathrm{e}^{ \pm\left(\lambda_{R_{ \pm}}-\mu_{R_{ \pm}}\right)} T^{-} \otimes T^{+}+\mathrm{e}^{\mp\left(\lambda_{R_{ \pm}}-\mu_{R_{ \pm}}\right)} T^{+} \otimes T^{-}\right\} \delta(x-y) .
\end{aligned}
$$

Similarly, the following can be obtained

$$
\begin{aligned}
& (r-s)^{R_{ \pm}}\left(\lambda_{R_{ \pm}}, \mu_{R_{ \pm}}\right) \delta(x-y) \\
= & \frac{1}{\sinh \left(\lambda_{R_{ \pm}}-\mu_{R_{ \pm}}\right)} \frac{\sinh 2 \alpha \sinh ^{2} \mu_{R_{ \pm}}}{2 \sinh \left(\alpha+\beta+\mu_{R_{ \pm}}\right) \sinh \left(\alpha-\beta-\mu_{R_{ \pm}}\right)} \\
& \times\left\{\left[\cosh \left(\lambda_{R_{ \pm}}-\mu_{R_{ \pm}}\right)+\tanh \beta \sinh \left(\lambda_{R_{ \pm}}-\mu_{R_{ \pm}}\right)\right] T^{3} \otimes T^{3}\right. \\
& \left.\quad+\mathrm{e}^{ \pm\left(\lambda_{R_{ \pm}}-\mu_{R_{ \pm}}\right)} T^{-} \otimes T^{+}+\mathrm{e}^{\mp\left(\lambda_{R_{ \pm}}-\mu_{R_{ \pm}}\right)} T^{+} \otimes T^{-}\right\} \delta(x-y) .
\end{aligned}
$$

Thus the resulting $r / s$-matrices for the reduced right Lax pairs are

$$
\begin{aligned}
& r^{R_{ \pm}}\left(\lambda_{R_{ \pm}}, \mu_{R_{ \pm}}\right)= \frac{h_{\alpha, \beta}^{R}\left(\lambda_{R_{ \pm}}\right)+h_{\alpha, \beta}^{R}\left(\mu_{R_{ \pm}}\right)}{2 \sinh \left(\lambda_{R_{ \pm}}-\mu_{R_{ \pm}}\right)} \\
& \times\left[\mathrm{e}^{ \pm\left(\lambda_{R_{ \pm}}-\mu_{R_{ \pm}}\right)} T^{-} \otimes T^{+}+\mathrm{e}^{\mp\left(\lambda_{R_{ \pm}}-\mu_{R_{ \pm}}\right)} T^{+} \otimes T^{-}\right. \\
&\left.\quad+\cosh \left(\lambda_{R_{ \pm}}-\mu_{R_{ \pm}}\right) T^{3} \otimes T^{3}\right] \\
& \\
&-\frac{\left(h_{\alpha, \beta}^{R}\left(\lambda_{R_{ \pm}}\right)-h_{\alpha, \beta}^{R}\left(\mu_{R_{ \pm}}\right)\right)}{2} \tanh \beta T^{3} \otimes T^{3},
\end{aligned}
$$




$$
\begin{aligned}
s^{R_{ \pm}}\left(\lambda_{R_{ \pm}}, \mu_{R_{ \pm}}\right)= & \frac{h_{\alpha, \beta}^{R}\left(\lambda_{R_{ \pm}}\right)-h_{\alpha, \beta}^{R}\left(\mu_{R_{ \pm}}\right)}{2 \sinh \left(\lambda_{R_{ \pm}}-\mu_{R_{ \pm}}\right)} \\
& \times\left[\mathrm{e}^{ \pm\left(\lambda_{R_{ \pm}}-\mu_{R_{ \pm}}\right)} T^{-} \otimes T^{+}+\mathrm{e}^{\mp\left(\lambda_{R_{ \pm}}-\mu_{R_{ \pm}}\right)} T^{+} \otimes T^{-}\right. \\
\left.\quad+\cosh \left(\lambda_{R_{ \pm}}-\mu_{R_{ \pm}}\right) T^{3} \otimes T^{3}\right] & \\
& -\frac{\left(h_{\alpha, \beta}^{R}\left(\lambda_{R_{ \pm}}\right)+h_{\alpha, \beta}^{R}\left(\mu_{R_{ \pm}}\right)\right)}{2} \tanh \beta T^{3} \otimes T^{3}
\end{aligned}
$$

The $r / s$-matrices for the right Lax pair (4.38) are obtained from these $r / s$-matrices for the reduced right Lax pairs by taking the isomorphism of $s l(2)$ (5.24) into account.

\section{The degenerate limits}

So far, it has been shown that $U(1)_{\mathrm{R}}$ is enhanced to $q$-deformed $s u(2)_{\mathrm{R}}$. Then let us argue the $q \rightarrow 1$ limit in which $q$-deformed $s u(2)_{\mathrm{R}}$ degenerates to the original $s u(2)_{\mathrm{R}}$.

In fact, there are two kinds of the $q \rightarrow 1$ limit, which are referred to as the degenerate limits. Since the $q$-parameter is written as $q=\mathrm{e}^{\gamma}$, the limits are specified by the condition, $\gamma=0$. From (4.19), this condition is equivalent to

$$
\alpha=0 \quad \text { or } \quad \alpha=\frac{\pi i}{2} \text {. }
$$

In the following, we will argue each of the limits.

\section{$6.1 \alpha=0$}

We begin with the $\alpha \rightarrow 0$ limit. Recall the relation between the original parameters $(C, K)$ and the parameters $(\alpha, \beta)$, (4.5) . Due to the relation and the finiteness of the original parameters, the condition $\alpha=0$ requires the condition $\beta=0$.

Let us rescale $\alpha$ and $\beta$ as

$$
\alpha \rightarrow \epsilon \alpha, \quad \beta \rightarrow \epsilon \beta,
$$

and take the $\epsilon \rightarrow 0$ limit. Then the relation (4.5) is reduced to the following:

$$
K=\frac{\beta}{\alpha}, \quad C=0, \quad A=0 .
$$

Thus this limit reproduces the undeformed $S U(2)$ WZNW models. 
Next we consider the limit for the right Lax pair. Let us perform the redefinition of spectral parameter,

$$
\lambda_{R} \rightarrow \epsilon \alpha \lambda_{R}-\epsilon \beta
$$

and take the $\epsilon \rightarrow 0$ limit. Then the Lax pair is evaluated as

$$
L_{ \pm}^{R}\left(x ; \lambda_{R}\right)=-\frac{1 \pm K}{1 \pm \lambda_{R}}\left[T^{+} J_{ \pm}^{-}+T^{-} J_{ \pm}^{+}+T^{3} J_{ \pm}^{3}\right]
$$

The same procedure gives rise to the $r / s$-matrices,

$$
\begin{aligned}
& r^{R}\left(\lambda_{R}, \mu_{R}\right)=\frac{h_{0}^{R}\left(\lambda_{R}\right)+h_{0}^{R}\left(\mu_{R}\right)}{2\left(\lambda_{R}-\mu_{R}\right)}\left[T^{-} \otimes T^{+}+T^{+} \otimes T^{-}+T^{3} \otimes T^{3}\right], \\
& s^{R}\left(\lambda_{R}, \mu_{R}\right)=\frac{h_{0}^{R}\left(\lambda_{R}\right)-h_{0}^{R}\left(\mu_{R}\right)}{2\left(\lambda_{R}-\mu_{R}\right)}\left[T^{-} \otimes T^{+}+T^{+} \otimes T^{-}+T^{3} \otimes T^{3}\right] .
\end{aligned}
$$

Here the function $h_{0}^{R}(\lambda)$ is given by

$$
h_{0}^{R}(\lambda)=\frac{(\lambda-K)^{2}}{1-\lambda^{2}} .
$$

Since $\gamma^{ \pm}=0$ and $A=0$, two sets of non-local currents $j_{\mu}^{R, \pm}$ and $\widetilde{j}_{\mu}^{R, \pm}$ degenerate into

$$
j_{\mu}^{R, \pm}=\widetilde{j}_{\mu}^{R, \pm}=-\left(J_{\mu}^{ \pm}+K \epsilon_{\mu \nu} J^{ \pm, \nu}\right) \equiv \mathcal{J}_{\mu}^{R, \pm}
$$

For later convenience, let us introduce the following matrix valued conserved current:

$$
\mathcal{J}_{\mu}^{R}=T^{+} \mathcal{J}_{\mu}^{R,-}+T^{-} \mathcal{J}_{\mu}^{R,+}+T^{3} \mathcal{J}_{\mu}^{R, 3}
$$

where the $T^{3}$ component $\mathcal{J}_{\mu}^{R, 3}$ is defined as

$$
\mathcal{J}_{\mu}^{R, 3} \equiv j_{\mu}^{R, 3}=-\left(J_{\mu}^{3}+K \epsilon_{\mu \nu} J^{3, \nu}\right)
$$

It is an easy task to show that this conserved current satisfies the flatness condition:

$$
\epsilon^{\mu \nu}\left(\partial_{\mu} \mathcal{J}_{\nu}^{R}-\mathcal{J}_{\mu}^{R} \mathcal{J}_{\nu}^{R}\right)=0
$$

and the right Lax pair can be written in terms of the conserved current $\mathcal{J}_{\mu}^{R}$ as

$$
L_{ \pm}^{R}\left(x ; \lambda_{R}\right)=\frac{\mathcal{J}_{ \pm}^{R}}{1 \pm \lambda_{R}}
$$


Then it is possible to construct an infinite number of conserved charges from $\mathcal{J}_{\mu}^{R}$, by following the BIZZ construction [8]:

$$
\begin{aligned}
& \mathcal{Y}_{(0)}^{R, a}=\int_{-\infty}^{\infty} d x \mathcal{J}_{t}^{R, a}(x) \\
& \mathcal{Y}_{(1)}^{R, a}=\frac{1}{4} \int_{-\infty}^{\infty} d x \int_{-\infty}^{\infty} d y \varepsilon^{a}{ }_{b c} \epsilon(x-y) \mathcal{J}_{t}^{R . b}(x) \mathcal{J}_{t}^{R . c}(y)-\int_{-\infty}^{\infty} d x \mathcal{J}_{x}^{R . a}(x),
\end{aligned}
$$

The current algebra for $\mathcal{J}_{\mu}^{R, a}$ is given by

$$
\begin{aligned}
& \left\{\mathcal{J}_{t}^{R, a}(x), \mathcal{J}_{t}^{R, b}(y)\right\}_{\mathrm{P}}=\varepsilon^{a b}{ }_{c} \mathcal{J}_{t}^{R, c}(x)+2 K \delta^{a b} \partial_{x} \delta(x-y), \\
& \left\{\mathcal{J}_{t}^{R, a}(x), \mathcal{J}_{x}^{R, b}(y)\right\}_{\mathrm{P}}=\varepsilon^{a b}{ }_{c} \mathcal{J}_{x}^{R, c}(x)+\left(1+K^{2}\right) \delta^{a b} \partial_{x} \delta(x-y), \\
& \left\{\mathcal{J}_{x}^{R, a}(x), \mathcal{J}_{x}^{R, b}(y)\right\}_{\mathrm{P}}=-K^{2} \varepsilon^{a b}{ }_{c} \mathcal{J}_{t}^{R, c}(x)+2 K \varepsilon^{a b}{ }_{c} \mathcal{J}_{x}^{R, c}(x) \delta(x-y) \\
& +2 K \delta^{a b} \partial_{x} \delta(x-y) \text {. }
\end{aligned}
$$

With this current algebra, one can show that the algebra formed by a set of $\mathcal{Y}_{(n)}^{R}$ is Yangian $\mathcal{Y}\left(s u(2)_{\mathrm{L}}\right)$ with an appropriate regularization of non-ultra local terms.

\section{$6.2 \alpha=\pi i / 2$}

The other degenerate limit is next considered. Again, due to the relation (4.5) and the finiteness of $(C, K)$, the condition $\alpha=\pi i / 2$ implies that $\beta=\pi i / 2$. So it is useful to redefine $\alpha$ and $\beta$ as

$$
\alpha \rightarrow \frac{\pi i}{2}+\epsilon \alpha, \quad \beta \rightarrow \frac{\pi i}{2}+\epsilon \beta
$$

The $\epsilon \rightarrow 0$ limit with the redefinition leads to the following relations,

$$
K=\frac{\beta}{\alpha}, \quad C=\frac{\beta^{2}}{\alpha^{2}}-1, \quad A=0 .
$$

This limit describes the points specified by $C=K^{2}-1$. As in the case with $\alpha=0$, the coefficient of the improvement term vanishes, $A=0$. At the points, the effect of the squashing parameter $C$ is canceled by the Wess-Zumino term. As a result, the right description becomes isotropic even though the metric of target space is deformed.

To see the right Lax pair at the points, the spectral parameter should be redefined as

$$
\lambda_{R} \quad \rightarrow \quad \epsilon \alpha \lambda_{R}-\epsilon \beta
$$


Then the $\epsilon \rightarrow 0$ limit leads to the following expression of the Lax pair,

$$
L_{ \pm}^{R}\left(x ; \lambda_{R}\right)=-\frac{1 \pm K}{1 \pm \lambda_{R}}\left[T^{+} J_{ \pm}^{-}+T^{-} J_{ \pm}^{+}+\left(1 \mp K \pm \lambda_{R}\right) T^{3} J_{ \pm}^{3}\right] .
$$

Similarly, the $r / s$-matrices are obtained as

$$
\begin{aligned}
r^{R}\left(\lambda_{R}, \mu_{R}\right)= & \frac{h_{\pi i / 2}^{R}\left(\lambda_{R}\right)+h_{\pi i / 2}^{R}\left(\mu_{R}\right)}{2\left(\lambda_{R}-\mu_{R}\right)}\left[T^{-} \otimes T^{+}+T^{+} \otimes T^{-}+T^{3} \otimes T^{3}\right] \\
& -\frac{h_{\pi i / 2}^{R}\left(\lambda_{R}\right)-h_{\pi i / 2}^{R}\left(\mu_{R}\right)}{2 K} T^{3} \otimes T^{3}, \\
s^{R}\left(\lambda_{R}, \mu_{R}\right)= & \frac{h_{\pi i / 2}^{R}\left(\lambda_{R}\right)-h_{\pi i / 2}^{R}\left(\mu_{R}\right)}{2\left(\lambda_{R}-\mu_{R}\right)}\left[T^{-} \otimes T^{+}+T^{+} \otimes T^{-}+T^{3} \otimes T^{3}\right] \\
& -\frac{h_{\pi i / 2}^{R}\left(\lambda_{R}\right)+h_{\pi i / 2}^{R}\left(\mu_{R}\right)}{2 K} T^{3} \otimes T^{3},
\end{aligned}
$$

where the function $h_{\pi i / 2}^{R}(\lambda)$ is defined as

$$
h_{\pi i / 2}^{R}(\lambda) \equiv \frac{(\lambda-K)^{2}}{1-\lambda^{2}}
$$

In this limit, the following relations are satisfied,

$$
\gamma^{ \pm}=-\gamma^{\mp}= \pm i / K, \quad A=0 .
$$

Then $\widetilde{j}_{\mu}^{R, \pm}$ coincide with $j_{\mu}^{R, \pm}$ as

$$
j_{\mu}^{R, \pm}=\widetilde{j}_{\mu}^{R, \pm}=-\mathrm{e}^{ \pm i \chi / K}\left(J^{ \pm}+K \epsilon_{\mu \nu} J^{ \pm, \nu}\right) \equiv \mathcal{J}_{\mu}^{R, \pm}
$$

Note that $\mathcal{J}_{\mu}^{R, \pm}$ remain non-local currents, in contrast to the $\alpha=0$ case. It is useful to define a matrix valued conserved current as

$$
\mathcal{J}_{\mu}^{R}=T^{+} \mathcal{J}_{\mu}^{R,-}+T^{-} \mathcal{J}_{\mu}^{R,+}+T^{3} \mathcal{J}_{\mu}^{R, 3},
$$

where the $T^{3}$ component $\mathcal{J}_{\mu}^{R, 3}$ is defined as

$$
\mathcal{J}_{\mu}^{R, 3} \equiv-j_{\mu}^{R, 3}=\left(K^{2} J_{\mu}^{3}+K \epsilon_{\mu \nu} J^{3, \nu}\right)
$$

Here the sign of $j_{\mu}^{R, 3}$ is flipped for later convenience. Although $\mathcal{J}_{\mu}^{R}$ is non-local, it satisfies the flatness condition,

$$
\epsilon^{\mu \nu}\left(\partial_{\mu} \mathcal{J}_{\nu}^{R}-\mathcal{J}_{\mu}^{R} \mathcal{J}_{\nu}^{R}\right)=0
$$


Thus an infinite number of conserved charges can be constructed from $\mathcal{J}_{\mu}^{R}$ like

$$
\begin{aligned}
& \mathcal{Y}_{(0)}^{R, a}=\int_{-\infty}^{\infty} d x \mathcal{J}_{t}^{R, a}(x) \\
& \mathcal{Y}_{(1)}^{R, a}=\frac{1}{4} \int_{-\infty}^{\infty} d x \int_{-\infty}^{\infty} d y \varepsilon^{a}{ }_{b c} \epsilon(x-y) \mathcal{J}_{t}^{R . b}(x) \mathcal{J}_{t}^{R . c}(y)-\int_{-\infty}^{\infty} d x \mathcal{J}_{x}^{R . a}(x),
\end{aligned}
$$

Again, the higher-level charges $\mathcal{Y}_{(n \geq 2)}^{R}$ are obtained recursively by the BIZZ procedure [8].

In addition, another Lax pair can be obtained from the flat current $\mathcal{J}_{\mu}^{R}$ as

$$
\mathcal{L}_{ \pm}^{R}\left(\lambda_{R}\right)=\frac{\mathcal{J}_{ \pm}^{R}}{1 \pm \lambda_{R}}
$$

where we use the same spectral parameter $\lambda_{R}$, for later convenience. This Lax pair is non-local and hence it is different from the Lax pair (6.18). However there exists a gauge transformation which connect the non-local Lax pair (6.26) to the right Lax pair (6.18) .

Let us find an appropriate gauge function $\|$. An important observation is that, at $\lambda_{R}=\infty$, the right Lax pair (6.18) does not vanish but approaches to a finite quantity as follows:

$$
L_{ \pm}^{R}\left(\lambda_{R}=\infty\right)=-T^{3}(1 \pm K) J_{ \pm}^{3}=-T^{3} \partial_{ \pm}\left[\frac{\chi}{K}\right]
$$

The gauge transformation must be chosen to eliminate this finite part because the non-local Lax pair (6.26) vanishes at $\lambda_{R}=\infty$. Thus the gauge transformation is generated by

$$
F(x)=\mathrm{e}^{-T^{3} \frac{\chi(x)}{K}}
$$

Note that a constant $S U(2)$ element may be multiplied from the right and thus the function $F(x)$ is not determined uniquely. However, the choice in (6.28) is appropriate to relate the right Lax pair (6.18) to the non-local Lax pair (6.26), as we will see just below.

By performing a non-local gauge transformation generated by $F(x)$ in (6.28), the right Lax pair (6.18) can be rewritten as

$$
\begin{aligned}
L_{ \pm}^{R}\left(\lambda_{R}\right) \rightarrow\left[L_{ \pm}^{R}\left(\lambda_{R}\right)\right]^{F} & =F^{-1} L_{ \pm}^{R}\left(\lambda_{R}\right) F-F^{-1} \partial_{ \pm} F \\
& =\frac{\mathcal{J}_{ \pm}^{R}}{1 \pm \lambda_{R}}=\mathcal{L}_{ \pm}^{R}\left(\lambda_{R}\right)
\end{aligned}
$$

\footnotetext{
\| The strategy is similar to the one to consider the Jordanian twists in the Schrödinger sigma models [29].
} 
According to the gauge transformation, the monodromy matrix is also transformed,

$$
\begin{aligned}
\mathcal{U}^{R}\left(\lambda_{R}\right)=\left[U^{R}\left(\lambda_{R}\right)\right]^{F} & =F^{-1}(x=\infty) U^{R}\left(\lambda_{R}\right) F(x=-\infty) \\
& =\mathrm{e}^{-\frac{T^{3} Q^{R, 3}}{2 K}} U^{R}\left(\lambda_{R}\right) \mathrm{e}^{-\frac{T^{3} Q^{R, 3}}{2 K}} .
\end{aligned}
$$

This relation may be interpreted as a classical analogue of the Reshetikhin twist [39]. It is interesting to reveal the relation to the quantum twist from the viewpoint of the mathematical formulation.

In addition, it is worth to note that the flat and conserved current $\mathcal{J}_{\mu}^{R}$ can be written as

$$
\mathcal{J}_{\mu}^{R}=-\left(G^{-1} \partial_{\mu} G+K \epsilon_{\mu \nu} G^{-1} \partial^{\nu} G\right), \quad G=g F
$$

Then the current algebra of $\mathcal{J}_{\mu}^{R, a}$ is given by

$$
\begin{aligned}
& \left\{\mathcal{J}_{t}^{R, a}(x), \mathcal{J}_{t}^{R, b}(y)\right\}_{\mathrm{P}}=\varepsilon^{a b}{ }_{c} \mathcal{J}_{t}^{R, c}(x)+2 K \delta^{a b} \partial_{x} \delta(x-y), \\
& \left\{\mathcal{J}_{t}^{R, a}(x), \mathcal{J}_{x}^{R, b}(y)\right\}_{\mathrm{P}}=\varepsilon^{a b}{ }_{c} \mathcal{J}_{x}^{R, c}(x)+\left(1+K^{2}\right) \delta^{a b} \partial_{x} \delta(x-y), \\
& \left\{\mathcal{J}_{x}^{R, a}(x), \mathcal{J}_{x}^{R, b}(y)\right\}_{\mathrm{P}}=-K^{2} \varepsilon^{a b}{ }_{c} \mathcal{J}_{t}^{R, c}(x)+2 K \varepsilon^{a b}{ }_{c} \mathcal{J}_{x}^{R, c}(x) \delta(x-y) \\
& +2 K \delta^{a b} \partial_{x} \delta(x-y) \text {. }
\end{aligned}
$$

Again, the infinite-dimensional algebra of $\mathcal{Y}_{(n)}^{R}$ is $\mathcal{Y}\left(s u(2)_{\mathrm{R}}\right)$.

\section{Conclusion and Discussion}

We have considered the classical integrable structure of the squashed WZNW models based on an infinite-dimensional extension of $U(1)_{\mathrm{R}}$. The system contains two deformation parameters. The one is provided by the coefficient of the Wess-Zumino term. The other is the squashing parameter of target space. We have constructed an anisotropic Lax pair and computed the associated $r / s$-matrices, which may be regarded as one-parameter deformations of the results without the Wess-Zumino term [25, 26].

Due to the presence of the Wess-Zumino term, an infinite-dimensional extension of $s u(2)_{\mathrm{R}}$ is given by a deformation of the standard quantum affine algebra, which contain two parameters. The deformed algebra contains two sets of $q$-deformed $s u(2)_{\mathrm{R}}$ and the tower structure of the conserved charges is quite similar to that of the standard quantum affine algebra. The only modification appears in the coefficient of the Poisson bracket. It also changes the 
classical $q$-Serre relations. The resulting algebra seems likely to be the classical analogue of a two-parameter quantum toroidal algebra [34]. However, it is not sure so far for this point because it seems that the first realization of the algebra has not been constructed yet.

The left-right duality has also been revealed by showing that the left Lax pairs are gaugeequivalent to the right Lax pair with the relation between the spectral parameters and the isomorphism of the $s l(2)$ algebra. This is a generalization of the duality in the case without the Wess-Zumino term. The right Lax pair can also be decomposed to a pair of the reduced right Lax pairs, each of which is equivalent to the corresponding left Lax pair.

In addition, two degenerate limits have been considered. They are realized at the points $C=0$ (corresponding to $\alpha=\beta=0$ ) and the points $C=K^{2}-1$ (corresponding to $\alpha=\beta=\pi i / 2)$. In the former case, the target space is undeformed and the right description becomes the isotropic. In the latter case, while the metric of the target space is still squashed, the effect of the squashing is compensated by taking an appropriate value of the coefficient of the Wess-Zumino term, so that the right description becomes the isotropic.

In each of the degenerate limits, we have constructed an $S U(2)_{\mathrm{R}}$ conserved current satisfying the flatness condition. With this current, another Lax pair can be constructed. For the later case, the construction is more involved and a non-local gauge-transformation is needed. In addition, Yangian generators have also been constructed.

There are some open issues. It would be interesting to study the fast-moving limit [40] of the squashed WZNW models (For squashed $\mathrm{S}^{3}$ and warped $\mathrm{AdS}_{3}$ see [41] and [42], respectively). Some applications of the results may be considered in the context of string theory. In this direction, for a deformation of $\mathrm{AdS}_{3} / \mathrm{CFT}_{2}$, see an earlier paper [43]. For warped $\mathrm{AdS}_{3}$ and squashed $\mathrm{S}^{3}$ geometries in string theory, for example, see [44 48].

We hope that some applications of the deformed quantum affine algebra would be found and be a key ingredient in studying the integrability of the AdS/CFT correspondence.

\section{Acknowledgment}

We would like to thank Takuya Matsumoto for useful discussions. The work of IK was supported by the Japan Society for the Promotion of Science (JSPS) . 


\section{Appendix}

\section{A The Poisson brackets of $J_{\mu}^{a}$ and $j_{\mu}^{L_{ \pm}}$}

The Poisson brackets of $J_{\mu}^{a}$ play an important role in computing the current algebra of $j_{\mu}^{L_{ \pm}}$. The computation is straightforward but very messy. In order to use the canonical Poisson brackets of the dynamical variables, the computation is described in terms of angle variables. Then, by using the Poisson brackets, the current algebra of $j_{\mu}^{L_{ \pm}}$is computed.

\section{A.1 The Poisson brackets of $J_{\mu}^{a}$}

The classical action (2.16) is composed of the two parts $S=S_{\sigma M}+S_{W Z}$. Each part is expressed in terms of the angle variables $(\theta, \phi, \psi)$ as follows:

$$
\begin{aligned}
S_{\sigma M}=\frac{1}{2 \lambda^{2}} \int_{-\infty}^{\infty} d t \int_{-\infty}^{\infty} d x\left[\dot{\theta}^{2}+\sin ^{2} \theta \dot{\phi}^{2}+(1+C)(\dot{\psi}+\cos \theta \dot{\phi})^{2}\right. \\
\left.\quad-\theta^{\prime 2}-\sin ^{2} \theta \phi^{\prime 2}-(1+C)\left(\psi^{\prime}+\cos \theta \phi^{\prime}\right)^{2}\right] \\
S_{W Z}=-\frac{n}{8 \pi} \int_{-\infty}^{\infty} d t \int_{-\infty}^{\infty} d x\left(\cos \theta \dot{\phi} \psi^{\prime}-\cos \theta \dot{\psi} \phi^{\prime}\right)
\end{aligned}
$$

The symbols "dot" and "prime" denote the derivatives with respect to $t$ and $x$, respectively.

The conjugate momenta $\left(\Pi_{\theta}, \Pi_{\phi}, \Pi_{\psi}\right)$ for $(\theta, \phi, \psi)$ are given by

$$
\begin{aligned}
& \Pi_{\theta}=\dot{\theta} \\
& \Pi_{\phi}=\sin ^{2} \theta \dot{\phi}+(1+C) \cos \theta(\dot{\psi}+\cos \theta \dot{\phi})-K \cos \theta \psi^{\prime}, \\
& \Pi_{\psi}=(1+C)(\dot{\psi}+\cos \theta \dot{\phi})+K \cos \theta \phi^{\prime} .
\end{aligned}
$$

The "velocity" variables are

$$
\begin{aligned}
& \dot{\theta}=\widehat{\Pi}_{\theta} \\
& \dot{\phi}=\frac{1}{\sin ^{2} \theta} \widehat{\Pi}_{\phi}-\frac{\cos \theta}{\sin ^{2} \theta} \widehat{\Pi}_{\psi}, \\
& \dot{\psi}=\left(\frac{1}{1+C}-\frac{\cos ^{2} \theta}{\sin ^{2} \theta}\right) \widehat{\Pi}_{\psi}-\frac{\cos \theta}{\sin ^{2} \theta} \widehat{\Pi}_{\phi},
\end{aligned}
$$

where $\left(\widehat{\Pi}_{\theta}, \widehat{\Pi}_{\phi}, \widehat{\Pi}_{\psi}\right)$ are defined as

$$
\widehat{\Pi}_{\theta} \equiv \Pi_{\theta}, \quad \widehat{\Pi}_{\phi} \equiv \Pi_{\phi}+K \cos \theta \psi^{\prime}, \quad \widehat{\Pi}_{\psi} \equiv \Pi_{\psi}-K \cos \theta \phi^{\prime} .
$$




\begin{tabular}{c||c|c|c|c|c|c} 
& $\theta$ & $\phi$ & $\psi$ & $\widehat{\Pi}_{\theta}$ & $\widehat{\Pi}_{\phi}$ & $\widehat{\Pi}_{\psi}$ \\
\hline \hline$\theta$ & 0 & 0 & 0 & 1 & 0 & 0 \\
\hline$\phi$ & 0 & 0 & 0 & 0 & 1 & 0 \\
\hline$\psi$ & 0 & 0 & 0 & 0 & 0 & 1 \\
\hline$\widehat{\Pi}_{\theta}$ & -1 & 0 & 0 & 0 & $K \sin \theta \psi^{\prime}$ & $-K \sin \theta \phi^{\prime}$ \\
\hline$\widehat{\Pi}_{\phi}$ & 0 & -1 & 0 & $-K \sin \theta \psi^{\prime}$ & 0 & $K \sin \theta \theta^{\prime}$ \\
\hline$\widehat{\Pi}_{\psi}$ & 0 & 0 & -1 & $K \sin \theta \phi^{\prime}$ & $-K \sin \theta \theta^{\prime}$ & 0 \\
\hline
\end{tabular}

Table 2: The Poisson brackets of the angle variables and the canonical momenta.

Then the Poisson brackets of the canonical variables are summarized in Tab.2,

With the canonical variables, the components of $J$ are expressed as

$$
\begin{aligned}
J_{t}^{ \pm} & =\frac{1}{\sqrt{2}} \mathrm{e}^{\mp i \psi}\left( \pm i \widehat{\Pi}_{\theta}-\frac{1}{\sin \theta} \widehat{\Pi}_{\phi}+\frac{\cos \theta}{\sin \theta} \widehat{\Pi}_{\psi}\right), \\
J_{t}^{3} & =\frac{1}{1+C} \widehat{\Pi}_{\psi}, \\
J_{x}^{ \pm} & =\frac{1}{\sqrt{2}} \mathrm{e}^{\mp i \psi}\left( \pm i \theta^{\prime}-\sin \theta \phi^{\prime}\right), \\
J_{x}^{3} & =\psi^{\prime}+\cos \theta \phi^{\prime} .
\end{aligned}
$$

By using the Poisson brackets in Tab.2 and the expressions given in (A.5), the Poisson brackets of $J_{\mu}^{a}$ are computed like

$$
\begin{aligned}
& \left\{J_{t}^{ \pm}(x), J_{t}^{\mp}(y)\right\}_{\mathrm{P}}=\mp i\left[-(1+C) J_{t}^{3}+K J_{x}^{3}\right](x) \delta(x-y), \\
& \left\{J_{t}^{ \pm}(x), J_{t}^{3}(y)\right\}_{\mathrm{P}}= \pm i\left[-\frac{1}{1+C} J_{t}^{ \pm}+\frac{K}{1+C} J_{x}^{ \pm}\right](x) \delta(x-y), \\
& \left\{J_{t}^{ \pm}(x), J_{x}^{\mp}(y)\right\}_{\mathrm{P}}=\mp i\left[-J_{x}^{3}\right](x) \delta(x-y)+\partial_{x} \delta(x-y), \\
& \left\{J_{t}^{ \pm}(x), J_{x}^{3}(y)\right\}_{\mathrm{P}}= \pm i\left[-J_{x}^{ \pm}\right](x) \delta(x-y), \\
& \left\{J_{t}^{3}(x), J_{x}^{ \pm}(y)\right\}_{\mathrm{P}}=\mp i\left[-\frac{1}{1+C} J_{x}^{ \pm}\right](x) \delta(x-y), \\
& \left\{J_{t}^{3}(x), J_{x}^{3}(y)\right\}_{\mathrm{P}}=\frac{1}{1+C} \partial_{x} \delta(x-y), \\
& \left\{J_{x}^{ \pm}(x), J_{t}^{\mp}(y)\right\}_{\mathrm{P}}=\mp i\left[-J_{x}^{3}\right](x) \delta(x-y)+\partial_{x} \delta(x-y), \\
& \left\{J_{x}^{ \pm}(x), J_{t}^{3}(y)\right\}_{\mathrm{P}}= \pm i\left[-\frac{1}{1+C} J_{x}^{ \pm}\right](x) \delta(x-y), \\
& \left\{J_{x}^{3}(x), J_{t}^{ \pm}(y)\right\}_{\mathrm{P}}=\mp i\left[-J_{x}^{ \pm}\right](x) \delta(x-y),
\end{aligned}
$$




$$
\left\{J_{x}^{3}(x), J_{t}^{3}(y)\right\}_{\mathrm{P}}=\frac{1}{1+C} \partial_{x} \delta(x-y)
$$

Similarly, the Poisson brackets between $g$ and $J_{\mu}^{a}$ are evaluated as

$$
\begin{aligned}
& \left\{g(x), J_{t}^{ \pm}(y)\right\}_{\mathrm{P}}=g(x) T^{ \pm} \delta(x-y), \\
& \left\{g(x), J_{t}^{3}(y)\right\}_{\mathrm{P}}=\frac{1}{1+C} g(x) T^{3} \delta(x-y) .
\end{aligned}
$$

\section{A.2 The current algebra of $j_{\mu}^{L_{ \pm}}$}

To compute the current algebra of $j_{\mu}^{L_{ \pm}}$, it is convenient to rewrite $j_{\mu}^{L_{ \pm}}$in terms of $J$ as

$$
\begin{aligned}
j_{\mu}^{L_{ \pm}}= & g\left\{J_{\mu}-2 C \operatorname{tr}\left(T^{3} J_{\mu}\right) T^{3}-K \epsilon_{\mu \nu} J^{\nu} \mp A \epsilon_{\mu \nu}\left[J^{\nu}, T^{3}\right]\right\} g^{-1} \\
= & g\left\{T^{+}\left[J_{\mu}^{-}-(K \pm i A) \epsilon_{\mu \nu} J^{-, \nu}\right]+T^{-}\left[J_{\mu}^{+}-(K \mp i A) \epsilon_{\mu \nu} J^{+, \nu}\right]\right. \\
& \left.+T^{3}\left[(1+C) J_{\mu}^{3}-K \epsilon_{\mu \nu} J^{3, \nu}\right]\right\} g^{-1} .
\end{aligned}
$$

With the relations in (A.7) and (A.8), the current algebra is computed as

$$
\begin{aligned}
\left\{j_{t}^{L_{ \pm}, a}(x), j_{t}^{L_{ \pm}, b}(y)\right\}_{\mathrm{P}}= & \varepsilon^{a b}{ }_{c} j_{t}^{L_{ \pm}, c}(x) \delta(x-y)-2 K \delta^{a b} \partial_{x} \delta(x-y) \\
\left\{j_{t}^{L_{ \pm}, a}(x), j_{x}^{L_{ \pm}, b}(y)\right\}_{\mathrm{P}}= & \varepsilon^{a b}{ }_{c} j_{x}^{L_{ \pm}, c}(x) \delta(x-y)+\left(1+K^{2}+A^{2}\right) \delta^{a b} \partial_{x} \delta(x-y), \\
\left\{j_{x}^{L_{ \pm}, a}(x), j_{x}^{L_{ \pm}, b}(y)\right\}_{\mathrm{P}}= & -\left(K^{2}+A^{2}\right) \varepsilon^{a b}{ }_{c} j_{t}^{L_{ \pm}, c}(x) \delta(x-y) \\
& -2 K \varepsilon^{a b}{ }_{c} j_{x}^{L_{ \pm}, c}(x) \delta(x-y)-2 K \delta^{a b} \partial_{x} \delta(x-y) .
\end{aligned}
$$

In addition, the Poisson brackets between $g$ and $j_{\mu}^{L_{+}}$are also obtained as

$$
\begin{aligned}
\left\{g(x), j_{t}^{L_{+}, a}(y)\right\}_{\mathrm{P}}= & T^{a} g(x) \delta(x-y), \\
\left\{g(x), j_{x}^{L_{+}, a}(y)\right\}_{\mathrm{P}}= & \left\{-\frac{K}{1+C} T^{a}+A\left[T^{a}, g T^{3} g^{-1}\right]\right. \\
& \left.\quad+\frac{C K}{1+C}\left[\left[T^{a}, g T^{3} g^{-1}\right], g T^{3} g^{-1}\right]\right\} g(x) \delta(x-y) .
\end{aligned}
$$

\section{B A prescription to treat non-ultra local terms}

We present the computations of 1) Yangian, 2) $q$-deformed $s u(2)_{\mathrm{R}}$, and 3) a deformed quantum affine algebra. In particular, a prescription to treat non-ultra local terms is carefully described in each case. 


\section{B.1 Yangian $\mathcal{Y}\left(s u(2)_{\mathrm{L}}\right)$}

Let us first compute Yangian $\mathcal{Y}\left(s u(2)_{\mathrm{L}}\right)$. Non-ultra local terms appear in the computations of the Poisson brackets of $Q_{(n)}^{L_{ \pm}}$and hence we should be careful of the order of limits. The subscripts of $L,( \pm)$ are omitted for simplicity henceforth.

\section{The Poisson brackets at level 0}

The first is the Poisson brackets of the level 0 charges. The charges are regularized as

$$
Q_{(0)}^{L, a}\left(X_{0}, X_{1}\right)=\int_{-X_{0}}^{X_{1}} d x j_{t}^{L, a}(x)
$$

Then the Poisson brackets of the regularized charges are given by

$$
\left\{Q_{(0)}^{L, a}\left(X_{0}, X_{1}\right), Q_{(0)}^{L, b}\left(Y_{0}, Y_{1}\right)\right\}_{\mathrm{P}}=\int_{-X_{0}}^{X_{1}} d x \int_{-Y_{0}}^{Y_{1}} d y\left[-2 K \delta^{a b} \partial_{x} \delta(x-y)+\varepsilon^{a b}{ }_{c} j_{t}^{L, c}(x) \delta(x-y)\right] .
$$

The first term contains a derivative of the delta function, called a non-ultra local term. This term develops an ambiguity depending on the order of limits as follows:

$$
\begin{aligned}
& \left\{Q_{(0)}^{L, a}\left(X_{0}, X_{1}\right), Q_{(0)}^{L, b}\left(Y_{0}, Y_{1}\right)\right\}_{\mathrm{P}} \\
= & -2 K \delta^{a b}\left[\theta\left(X_{0}-Y_{0}\right)-\theta\left(X_{1}-Y_{1}\right)\right]+\varepsilon^{a b}{ }_{c} Q_{(0)}^{L, c}\left(\min \left(X_{0}, Y_{0}\right), \min \left(X_{1}, Y_{1}\right)\right) .
\end{aligned}
$$

In the following, we will not write down unambiguous terms explicitly as follows:

$$
\begin{aligned}
& \left\{Q_{(0)}^{L, a}\left(X_{0}, X_{1}\right), Q_{(0)}^{L, b}\left(Y_{0}, Y_{1}\right)\right\}_{\mathrm{P}} \\
= & -2 K \delta^{a b}\left[\theta\left(X_{0}-Y_{0}\right)-\theta\left(X_{1}-Y_{1}\right)\right]+(\text { no ambiguity }) .
\end{aligned}
$$

Note that this ambiguity arises due to the presence of the Wess-Zumino term. When $K=0$, there is no ambiguity at the level 0 as usual.

Now, one may follow a prescription proposed in [10],

$$
X_{0}=X_{1} \equiv X, \quad Y_{0}=Y_{1} \equiv Y
$$

so as to make the ambiguous term vanish. Then, by taking the limits $X \rightarrow \infty$ and $Y \rightarrow \infty$, the following is obtained,

$$
\left\{Q_{(0)}^{L, a}, Q_{(0)}^{L, b}\right\}_{\mathrm{P}}=\varepsilon^{a b}{ }_{c} Q_{(0)}^{L, c}
$$




\section{The Poisson brackets at level 1}

The next is to regularize the level 1 charges. The level 1 charges are regularized as

$$
Q_{(1)}^{L, a}\left(X, X^{\prime}, X^{\prime \prime}\right)=\frac{1}{4} \int_{-X}^{X} d x \int_{-X^{\prime}}^{X^{\prime}} d x^{\prime} \epsilon\left(x-x^{\prime}\right) \varepsilon_{b c}^{a} j_{t}^{L, b}(x) j_{t}^{L, c}\left(x^{\prime}\right)-\int_{-X^{\prime \prime}}^{X^{\prime \prime}} d x^{\prime \prime} j_{x}^{L, a}\left(x^{\prime \prime}\right) .
$$

The Poisson brackets of the regularized level 1 and level 0 charges are

$$
\begin{aligned}
& \left\{Q_{(1)}^{L, a}\left(X, X^{\prime}, X^{\prime \prime}\right), Q_{(0)}^{L, b}(Y)\right\}_{\mathrm{P}} \\
= & 2 K \varepsilon_{c}^{a b}\left[\theta(X-Y) Q_{(0)}^{L, c}\left(\min \left(X^{\prime}, Y\right)\right)+\theta\left(X^{\prime}-Y\right) Q_{(0)}^{L, c}(\min (X, Y))\right] \\
& +(\text { no ambiguity }) .
\end{aligned}
$$

Now the unambiguous terms are given by

$$
\varepsilon_{c}^{a b} Q_{(1)}^{L, c}
$$

and hence the ambiguous terms have to vanish. Thus the $Y \rightarrow \infty$ limit should be taken before the $X, X^{\prime}, X^{\prime \prime} \rightarrow \infty$ limits. As a result, the following is obtained,

$$
\left\{Q_{(1)}^{L, a}, Q_{(0)}^{L, b}\right\}_{\mathrm{P}}=\varepsilon_{c}^{a b} Q_{(1)}^{L, a}
$$

\section{The Poisson brackets at level 2}

The Poisson brackets of the regularized level 1 charges are computed here. Those give rise to the brackets at level 2,

$$
\begin{gathered}
\left\{Q_{(1)}^{L, a}\left(X, X^{\prime}, X^{\prime \prime}\right), Q_{(1)}^{L, b}\left(Y, Y^{\prime}, Y^{\prime \prime}\right)\right\}_{\mathrm{P}} \\
=-\frac{K}{8} \theta(X-Y) \int_{-X^{\prime}}^{X^{\prime}} d x^{\prime} \int_{-Y^{\prime}}^{Y^{\prime}} d y^{\prime} \\
\times\left[\epsilon\left(x^{\prime}+Y\right) \epsilon\left(y^{\prime}+Y\right)-\epsilon\left(x^{\prime}-Y\right) \epsilon\left(y^{\prime}-Y\right)\right] \\
\times\left[\delta^{a b} \delta_{c d} j_{t}^{L, c}\left(x^{\prime}\right) j_{t}^{L, d}\left(y^{\prime}\right)-j_{t}^{L, b}\left(x^{\prime}\right) j_{t}^{L, a}\left(y^{\prime}\right)\right] \\
-\frac{K}{8} \theta\left(X^{\prime}-Y\right) \int_{-X}^{X} d x \int_{-Y^{\prime}}^{Y^{\prime}} d y^{\prime} \\
\times\left[\epsilon(x+Y) \epsilon\left(y^{\prime}+Y\right)-\epsilon(x-Y) \epsilon\left(y^{\prime}-Y\right)\right] \\
\times\left[\delta^{a b} \delta_{c d} j_{t}^{L, c}(x) j_{t}^{L, d}\left(y^{\prime}\right)-j_{t}^{L, b}(x) j_{t}^{L, a}\left(y^{\prime}\right)\right] \\
-\frac{K}{8} \theta\left(X-Y^{\prime}\right) \int_{-X^{\prime}}^{X^{\prime}} d x^{\prime} \int_{-Y}^{Y} d y
\end{gathered}
$$




$$
\begin{gathered}
\times\left[\epsilon\left(x^{\prime}+Y^{\prime}\right) \epsilon\left(y+Y^{\prime}\right)-\epsilon\left(x^{\prime}-Y^{\prime}\right) \epsilon\left(y-Y^{\prime}\right)\right] \\
\times\left[\delta^{a b} \delta_{c d} j_{t}^{L, c}\left(x^{\prime}\right) j_{t}^{L, d}(y)-j_{t}^{L, b}\left(x^{\prime}\right) j_{t}^{L, a}(y)\right] \\
-\frac{K}{8} \theta\left(X^{\prime}-Y^{\prime}\right) \int_{-X}^{X} d x \int_{-Y}^{Y} d y \\
\times\left[\epsilon\left(x+Y^{\prime}\right) \epsilon\left(y+Y^{\prime}\right)-\epsilon\left(x-Y^{\prime}\right) \epsilon\left(y-Y^{\prime}\right)\right] \\
\times\left[\delta^{a b} \delta_{c d} j_{t}^{L, c}(x) j_{t}^{L, d}(y)-j_{t}^{L, b}(x) j_{t}^{L, a}(y)\right] \\
+\frac{(1+C)^{2}+K^{2}}{4(1+C)} \theta\left(X-Y^{\prime \prime}\right) \varepsilon^{a b}{ }_{c} \int_{-X^{\prime}}^{X^{\prime}} d x^{\prime}\left[\epsilon\left(x^{\prime}+Y^{\prime \prime}\right)-\epsilon\left(x^{\prime}-Y^{\prime \prime}\right)\right] j_{t}^{L, c}\left(x^{\prime}\right) \\
+\frac{(1+C)^{2}+K^{2}}{4(1+C)} \theta\left(X^{\prime}-Y^{\prime \prime}\right) \varepsilon^{a b} \int_{-X}^{X} d x\left[\epsilon\left(x+Y^{\prime \prime}\right)-\epsilon\left(x-Y^{\prime \prime}\right)\right] j_{t}^{L, c}(x) \\
+\frac{(1+C)^{2}+K^{2}}{4(1+C)} \theta\left(Y-X^{\prime \prime}\right) \varepsilon^{a b}{ }_{c} \int_{-Y^{\prime}}^{Y^{\prime}} d y^{\prime}\left[\epsilon\left(y^{\prime}+X^{\prime \prime}\right)-\epsilon\left(y^{\prime}-X^{\prime \prime}\right)\right] j_{t}^{L, c}\left(y^{\prime}\right) \\
+\frac{(1+C)^{2}+K^{2}}{4(1+C)} \theta\left(Y^{\prime}-X^{\prime \prime}\right) \varepsilon^{a b} \int_{-Y^{\prime}}^{Y} d y\left[\epsilon\left(y+X^{\prime \prime}\right)-\epsilon\left(y-X^{\prime \prime}\right)\right] j_{t}^{L, c}(y) \\
+(\text { no ambiguity). }
\end{gathered}
$$

By taking the $Y, Y^{\prime}, Y^{\prime \prime \prime} \rightarrow \infty$ limits before sending $X, X^{\prime}, X^{\prime \prime}$ to infinity, the following expression is obtained,

$$
\begin{aligned}
& \left\{Q_{(1)}^{L, a}\left(X, X^{\prime}, X^{\prime \prime}\right), Q_{(1)}^{L, b}\right\}_{\mathrm{P}} \\
= & \min _{Y, Y^{\prime}, Y^{\prime \prime} \rightarrow \infty}\left\{Q_{(1)}^{L, a}\left(X, X^{\prime}, X^{\prime \prime}\right), Q_{(1)}^{L, b}\left(Y, Y^{\prime}, Y^{\prime \prime}\right)\right\}_{\mathrm{P}} \\
= & \frac{(1+C)^{2}+K^{2}}{2(1+C)} \varepsilon^{a b}{ }_{c} \int_{-\infty}^{\infty} d y\left[\epsilon\left(y+X^{\prime \prime}\right)-\epsilon\left(y-X^{\prime \prime}\right)\right] j_{t}^{L, c}(y)+(\text { no ambiguity }) .
\end{aligned}
$$

Then the ambiguous terms are given by

$$
\frac{(1+C)^{2}+K^{2}}{1+C} \varepsilon^{a b}{ }_{c} Q_{(0)}^{L, c},
$$

by taking the $X, X^{\prime}, X^{\prime \prime} \rightarrow \infty$ limits.

On the other hand, since the unambiguous terms are represented by

$$
\varepsilon_{c}^{a b}\left[Q_{(2)}^{L, c}+\frac{1}{12}\left(Q_{(0)}^{L}\right)^{2} Q_{(0)}^{L, c}+2 K Q_{(1)}^{L, c}-\frac{(1+C)^{2}+K^{2}}{1+C} Q_{(0)}^{L, c}\right],
$$

the net result is

$$
\left\{Q_{(1)}^{L, a}, Q_{(1)}^{L, b}\right\}_{\mathrm{P}}=\varepsilon^{a b}\left[Q_{(2)}^{L, c}+\frac{1}{12}\left(Q_{(0)}^{L}\right)^{2} Q_{(0)}^{L, c}+2 K Q_{(1)}^{L, c}\right]
$$




\section{Serre relations}

The Serre-relations are finally considered. One of them is represented by

$$
\left\{Q_{(1)}^{L, 3},\left\{Q_{(1)}^{L,+}, Q_{(1)}^{L,-}\right\}_{\mathrm{P}}\right\}_{\mathrm{P}}=\frac{1}{4} Q_{(0)}^{L, 3}\left[Q_{(0)}^{L,+} Q_{(1)}^{L,-}-Q_{(0)}^{L,-} Q_{(1)}^{L,+}\right] .
$$

Let us first introduce the following quantity:

$$
\begin{aligned}
& \left\{Q_{(1)}^{L,+}, Q_{(1)}^{L,-}\right\}_{\mathrm{P}}\left(X, X^{\prime}, X^{\prime \prime}, X^{\prime \prime \prime}, X^{\prime \prime \prime \prime}, X^{\prime \prime \prime \prime \prime \prime}\right) \\
& =-\frac{i}{4} \int_{-X}^{X} d x \int_{-X^{\prime}}^{X^{\prime}} d x^{\prime} \int_{-X^{\prime \prime}}^{X^{\prime \prime}} d x^{\prime \prime} \epsilon\left(x-x^{\prime}\right) \epsilon\left(x-x^{\prime \prime}\right) \\
& \times\left[j_{t}^{L,+}(x) j_{t}^{L, 3}\left(x^{\prime}\right) j_{t}^{L,-}\left(x^{\prime \prime}\right)+j_{t}^{L,-}(x) j_{t}^{L, 3}\left(x^{\prime}\right) j_{t}^{L,+}\left(x^{\prime \prime}\right)\right. \\
& \left.+j_{t}^{L, 3}(x) j_{t}^{L, 3}\left(x^{\prime}\right) j_{t}^{L, 3}\left(x^{\prime \prime}\right)\right] \\
& -\frac{1}{2} \int_{-X^{\prime \prime \prime}}^{X^{\prime \prime \prime}} d x^{\prime \prime \prime} \int_{-X^{\prime \prime \prime \prime}}^{X^{\prime \prime \prime \prime}} d x^{\prime \prime \prime \prime} \epsilon\left(x^{\prime \prime \prime}-x^{\prime \prime \prime \prime}\right) \\
& \times\left[j_{t}^{L,+}\left(x^{\prime \prime \prime}\right) j_{x}^{L,-}\left(x^{\prime \prime \prime \prime}\right)-j_{t}^{L,-}\left(x^{\prime \prime \prime}\right) j_{x}^{L,+}\left(x^{\prime \prime \prime \prime}\right)\right] \\
& -i \int_{-X^{\prime \prime \prime \prime \prime \prime}}^{X^{\prime \prime \prime \prime \prime \prime}} d x^{\prime \prime \prime \prime \prime \prime} j_{t}^{L, 3}\left(x^{\prime \prime \prime \prime \prime \prime}\right) \\
& +K \int_{-X^{\prime \prime \prime}}^{X^{\prime \prime \prime}} d x^{\prime \prime \prime} \int_{-X^{\prime \prime \prime \prime}}^{X^{\prime \prime \prime \prime}} d x^{\prime \prime \prime \prime} \epsilon\left(x^{\prime \prime \prime}-x^{\prime \prime \prime \prime \prime}\right) j_{t}^{L,+}\left(x^{\prime \prime \prime}\right) j_{t}^{L,-}\left(x^{\prime \prime \prime \prime \prime}\right) \\
& +2 i K \int_{-X^{\prime \prime \prime \prime \prime \prime}}^{X^{\prime \prime \prime \prime \prime}} d x^{\prime \prime \prime \prime \prime \prime \prime} j_{x}^{L, 3}\left(x^{\prime \prime \prime \prime \prime \prime}\right) \text {. }
\end{aligned}
$$

Note that the regularized $Q_{(1)}^{L, 3}$ is given by

$$
Q_{(1)}^{L, 3}\left(Y, Y^{\prime}, Y^{\prime \prime}\right)=\frac{i}{2} \int_{-Y}^{Y} d y \int_{-Y^{\prime}}^{Y^{\prime}} d y^{\prime} \epsilon\left(y-y^{\prime}\right) j_{t}^{L,+}(y) j_{t}^{L,-}\left(y^{\prime}\right)-\int_{-Y^{\prime \prime}}^{Y^{\prime \prime}} d y^{\prime \prime} j_{x}^{L, 3}\left(y^{\prime \prime}\right) .
$$

By using the quantities in (B.15) and (B.16), the following relation is obtained,

$$
\begin{aligned}
& \left\{Q_{(1)}^{L, 3}\left(Y, Y^{\prime}, Y^{\prime \prime}\right),\left\{Q_{(1)}^{L,+}, Q_{(1)}^{L,-}\right\}_{\mathrm{P}}\left(X, X^{\prime}, X^{\prime \prime}, X^{\prime \prime \prime}, X^{\prime \prime \prime \prime}, X^{\prime \prime \prime \prime \prime \prime}\right)\right\}_{\mathrm{P}} \\
= & \frac{K}{2} \int_{-\min \left(X, X^{\prime \prime}, Y\right)}^{\min \left(X, X^{\prime \prime}, Y\right)} d x \int_{-X^{\prime}}^{X^{\prime}} d x^{\prime} \int_{-Y^{\prime}}^{Y^{\prime}} d y^{\prime} \epsilon\left(x-x^{\prime}\right) \epsilon\left(x-y^{\prime}\right) j_{t}^{L,+}(x) j_{t}^{L, 3}\left(x^{\prime}\right) j_{t}^{L,-}\left(y^{\prime}\right) \\
& -\frac{K}{2} \int_{-\min \left(X, X^{\prime \prime}, Y^{\prime}\right)}^{\min \left(X, X^{\prime \prime}, Y^{\prime}\right)} d x \int_{-X^{\prime}}^{X^{\prime}} d x^{\prime} \int_{-Y}^{Y} d y \epsilon\left(x-x^{\prime}\right) \epsilon(x-y) j_{t}^{L,-}(x) j_{t}^{L, 3}\left(x^{\prime}\right) j_{t}^{L,+}(y) \\
& +\frac{K}{2} \int_{-\min \left(X, X^{\prime}, Y^{\prime}\right)}^{\min \left(X, X^{\prime}, Y^{\prime}\right)} d x_{-X^{\prime}}^{X^{\prime \prime}} d x^{\prime \prime} \int_{-Y}^{Y} d y \epsilon\left(x^{\prime}-y\right) \epsilon\left(x^{\prime}-x^{\prime \prime}\right) j_{t}^{L, 3}\left(x^{\prime}\right) j_{t}^{L,-}\left(x^{\prime \prime}\right) j_{t}^{L,+}(y) \\
& +\frac{K}{2} \int_{-X^{\prime}}^{X^{\prime}} d x_{-\min \left(X, X^{\prime \prime}, Y^{\prime}\right)}^{\min \left(X, X^{\prime \prime}, Y^{\prime}\right)} d x_{-Y}^{\prime \prime} \int_{-Y}^{Y} \epsilon\left(x^{\prime \prime}-y\right) \epsilon\left(x^{\prime \prime}-x^{\prime}\right) j_{t}^{L,-}\left(x^{\prime \prime}\right) j_{t}^{L, 3}\left(x^{\prime}\right) j_{t}^{L,+}(y)
\end{aligned}
$$




$$
\begin{aligned}
& -\frac{K}{2} \int_{-\min \left(X, X^{\prime}, Y\right)}^{\min \left(X, X^{\prime}, Y\right)} d x^{\prime} \int_{-X^{\prime \prime}}^{X^{\prime \prime}} d x^{\prime \prime} \int_{-Y^{\prime}}^{Y^{\prime}} d y^{\prime} \epsilon\left(x^{\prime}-y^{\prime}\right) \epsilon\left(x^{\prime}-x^{\prime \prime}\right) j_{t}^{L, 3}\left(x^{\prime}\right) j_{t}^{L,+}\left(x^{\prime \prime}\right) j_{t}^{L,-}\left(y^{\prime}\right) \\
& -\frac{K}{2} \int_{-X^{\prime}}^{X^{\prime}} d x^{\prime} \int_{-\min \left(X, X^{\prime \prime}, Y\right)}^{\min \left(X, X^{\prime \prime}, Y\right)} d x^{\prime \prime} \int_{-Y^{\prime}}^{Y^{\prime}} d y^{\prime} \epsilon\left(x^{\prime \prime}-y^{\prime}\right) \epsilon\left(x^{\prime \prime}-x^{\prime}\right) j_{t}^{L,+}\left(x^{\prime \prime}\right) j_{t}^{L, 3}\left(x^{\prime}\right) j_{t}^{L,-}\left(y^{\prime}\right) \\
& +\frac{K}{4} \theta\left(Y-X^{\prime \prime}\right) \int_{-X}^{X} d x \int_{-X^{\prime}}^{X^{\prime}} d x^{\prime} \int_{-Y^{\prime}}^{Y^{\prime}} d y^{\prime} \epsilon\left(x-x^{\prime}\right) j_{t}^{L,+}(x) j_{t}^{L, 3}\left(x^{\prime}\right) j_{t}^{L,-}\left(y^{\prime}\right) \\
& \times\left[\epsilon\left(y^{\prime}+X^{\prime \prime}\right) \epsilon\left(x+X^{\prime \prime}\right)-\epsilon\left(y^{\prime}-X^{\prime \prime}\right) \epsilon\left(x-X^{\prime \prime}\right)\right] \\
& -\frac{K}{4} \theta\left(Y^{\prime}-X^{\prime \prime}\right) \int_{-X}^{X} d x \int_{-X^{\prime}}^{X^{\prime}} d x^{\prime} \int_{-Y}^{Y} d y \epsilon\left(x-x^{\prime}\right) j_{t}^{L,-}(x) j_{t}^{L, 3}\left(x^{\prime}\right) j_{t}^{L,+}(y) \\
& \times\left[\epsilon\left(y+X^{\prime \prime}\right) \epsilon\left(x+X^{\prime \prime}\right)-\epsilon\left(y-X^{\prime \prime}\right) \epsilon\left(x-X^{\prime \prime}\right)\right] \\
& -\frac{K}{4} \theta\left(Y^{\prime}-X\right) \int_{-X^{\prime}}^{X^{\prime}} d x^{\prime} \int_{-X^{\prime \prime}}^{X^{\prime \prime}} d x^{\prime \prime} \int_{-Y}^{Y} d y j_{t}^{L, 3}\left(x^{\prime}\right) j_{t}^{L,-}\left(x^{\prime \prime}\right) j_{t}^{L,+}(y) \\
& \times\left[\epsilon(y+X) \epsilon\left(x^{\prime}+X\right) \epsilon\left(x^{\prime \prime}+X\right)-\epsilon(y-X) \epsilon\left(x^{\prime}-X\right) \epsilon\left(x^{\prime \prime}-X\right)\right] \\
& -\frac{K}{4} \theta(Y-X) \int_{-X^{\prime}}^{X^{\prime}} d x^{\prime} \int_{-X^{\prime \prime}}^{X^{\prime \prime}} d x^{\prime \prime} \int_{-Y^{\prime}}^{Y^{\prime}} d y^{\prime} j_{t}^{L, 3}\left(x^{\prime}\right) j_{t}^{L,+}\left(x^{\prime \prime}\right) j_{t}^{L,-}\left(y^{\prime}\right) \\
& \times\left[\epsilon\left(y^{\prime}+X\right) \epsilon\left(x^{\prime}+X\right) \epsilon\left(x^{\prime \prime}+X\right)-\epsilon\left(y^{\prime}-X\right) \epsilon\left(x^{\prime}-X\right) \epsilon\left(x^{\prime \prime}-X\right)\right] \\
& +\frac{i}{2} \frac{(1+C)^{2}+K^{2}}{1+C} \int_{-\min \left(X^{\prime \prime \prime}, X^{\prime \prime \prime \prime}, Y\right)}^{\min \left(X^{\prime \prime \prime}, X^{\prime \prime \prime \prime}, Y\right)} d x^{\prime \prime \prime} \int_{-Y^{\prime}}^{Y^{\prime}} d y^{\prime} \epsilon\left(x^{\prime \prime \prime}-y^{\prime}\right) j_{t}^{L,+}\left(x^{\prime \prime \prime}\right) j_{t}^{L,-}\left(y^{\prime}\right) \\
& -\frac{i}{2} \frac{(1+C)^{2}+K^{2}}{1+C} \int_{-\min \left(X^{\prime \prime \prime}, X^{\prime \prime \prime \prime}, Y^{\prime}\right)}^{\min \left(X^{\prime \prime \prime}, X^{\prime \prime \prime \prime}, Y^{\prime}\right)} d x^{\prime \prime \prime} \int_{-Y}^{Y} d y \epsilon\left(x^{\prime \prime \prime}-y\right) j_{t}^{L,+}(y) j_{t}^{L,-}\left(x^{\prime \prime \prime}\right) \\
& +i K \int_{-\min \left(X^{\prime \prime \prime}, X^{\prime \prime \prime \prime}, Y^{\prime}\right)}^{\min \left(X^{\prime \prime \prime}, X^{\prime \prime \prime \prime}, Y^{\prime}\right)} d x^{\prime \prime \prime \prime \prime} \int_{-Y}^{Y} d y \epsilon\left(y-x^{\prime \prime \prime \prime}\right) j_{t}^{L,+}(y) j_{x}^{L,-}\left(x^{\prime \prime \prime \prime \prime}\right) \\
& -i K \int_{-\min \left(X^{\prime \prime \prime}, X^{\prime \prime \prime \prime}, Y\right)}^{\min \left(X^{\prime \prime \prime}, X^{\prime \prime \prime \prime}, Y\right)} d x^{\prime \prime \prime \prime \prime} \int_{-Y^{\prime}}^{Y^{\prime}} d y^{\prime} \epsilon\left(x^{\prime \prime \prime \prime \prime}-y^{\prime}\right) j_{x}^{L,+}\left(x^{\prime \prime \prime \prime}\right) j_{t}^{L,-}\left(y^{\prime}\right) \\
& -\frac{i}{2} \frac{(1+C)^{2}+K^{2}}{1+C} \int_{-\min \left(X, X^{\prime}, Y^{\prime \prime}\right)}^{\min \left(X, X^{\prime}, Y^{\prime \prime}\right)} d x \int_{-X^{\prime \prime}}^{X^{\prime \prime}} d x^{\prime \prime} \epsilon\left(x-x^{\prime \prime}\right) j_{t}^{L,+}(x) j_{t}^{L,-}\left(x^{\prime \prime}\right) \\
& -\frac{i}{2} \frac{(1+C)^{2}+K^{2}}{1+C} \int_{-\min \left(X, X^{\prime}, Y^{\prime \prime}\right)}^{\min \left(X, X^{\prime}, Y^{\prime \prime}\right)} d x \int_{-X^{\prime \prime}}^{X^{\prime \prime}} d x^{\prime \prime} \epsilon\left(x-x^{\prime \prime}\right) j_{t}^{L,-}(x) j_{t}^{L,+}\left(x^{\prime \prime}\right) \\
& +\frac{i}{2} \frac{(1+C)^{2}+K^{2}}{1+C} \int_{-\min \left(X, X^{\prime}, Y^{\prime \prime}\right)}^{\min \left(X, X^{\prime}, Y^{\prime \prime}\right)} d x^{\prime} \int_{-X^{\prime \prime}}^{X^{\prime \prime}} d x^{\prime \prime} \epsilon\left(x^{\prime}-x^{\prime \prime}\right) j_{t}^{L, 3}\left(x^{\prime}\right) j_{t}^{L, 3}\left(x^{\prime \prime}\right) \\
& +\frac{i}{2} \frac{(1+C)^{2}+K^{2}}{1+C} \int_{-X^{\prime}}^{X^{\prime}} d x^{\prime} \int_{-\min \left(X, X^{\prime \prime}, Y^{\prime \prime}\right)}^{\min \left(X, X^{\prime \prime}, Y^{\prime \prime}\right)} d x^{\prime \prime} \epsilon\left(x^{\prime \prime}-x^{\prime}\right) j_{t}^{L, 3}\left(x^{\prime \prime}\right) j_{t}^{L, 3}\left(x^{\prime}\right) \\
& -\frac{i}{2} \frac{(1+C)^{2}+K^{2}}{1+C} \int_{-\min \left(X, X^{\prime}, Y^{\prime \prime}\right)}^{\min \left(X, X^{\prime}, Y^{\prime \prime}\right)} d x \int_{-X^{\prime \prime}}^{X^{\prime \prime}} d x^{\prime \prime} \epsilon\left(x-x^{\prime \prime}\right) j_{t}^{L, 3}(x) j_{t}^{L, 3}\left(x^{\prime \prime}\right) \\
& -\frac{i}{2} \frac{(1+C)^{2}+K^{2}}{1+C} \int_{-\min \left(X, X^{\prime \prime}, Y^{\prime \prime}\right)}^{\min \left(X, X^{\prime \prime}, Y^{\prime \prime}\right)} d x \int_{-X^{\prime}}^{X^{\prime}} d x^{\prime} \epsilon\left(x-x^{\prime}\right) j_{t}^{L, 3}(x) j_{t}^{L, 3}\left(x^{\prime}\right)
\end{aligned}
$$




$$
\begin{aligned}
& +\frac{i}{4} \frac{(1+C)^{2}+K^{2}}{1+C} \theta\left(Y-X^{\prime \prime \prime \prime}\right) \int_{-X^{\prime \prime \prime}}^{X^{\prime \prime \prime}} d x^{\prime \prime \prime} \int_{-Y^{\prime}}^{Y^{\prime}} d y^{\prime} j_{t}^{L,+}\left(x^{\prime \prime \prime}\right) j_{t}^{L,-}\left(y^{\prime}\right) \\
& \times\left[\epsilon\left(x^{\prime \prime \prime}+X^{\prime \prime \prime \prime}\right) \epsilon\left(y^{\prime}+X^{\prime \prime \prime \prime}\right)-\epsilon\left(x^{\prime \prime \prime}-X^{\prime \prime \prime \prime}\right) \epsilon\left(y^{\prime}-X^{\prime \prime \prime \prime}\right)\right] \\
& +\frac{i}{4} \frac{(1+C)^{2}+K^{2}}{1+C} \theta\left(Y^{\prime}-X^{\prime \prime \prime \prime}\right) \int_{-X^{\prime \prime \prime}}^{X^{\prime \prime \prime}} d x^{\prime \prime \prime} \int_{-Y}^{Y} d y j_{t}^{L,-}\left(x^{\prime \prime \prime}\right) j_{t}^{L,+}(y) \\
& \times\left[\epsilon\left(x^{\prime \prime \prime}+X^{\prime \prime \prime \prime}\right) \epsilon\left(y+X^{\prime \prime \prime \prime}\right)-\epsilon\left(x^{\prime \prime \prime}-X^{\prime \prime \prime \prime}\right) \epsilon\left(y-X^{\prime \prime \prime \prime}\right)\right] \\
& -\frac{i}{2} K \theta\left(Y^{\prime}-X^{\prime \prime \prime}\right) \int_{-X^{\prime \prime \prime \prime}}^{X^{\prime \prime \prime \prime}} d x^{\prime \prime \prime \prime} \int_{-Y}^{Y} d y j_{x}^{L,-}\left(x^{\prime \prime \prime \prime \prime}\right) j_{t}^{L,+}(y) \\
& \times\left[\epsilon\left(x^{\prime \prime \prime \prime}+X^{\prime \prime \prime}\right) \epsilon\left(y+X^{\prime \prime \prime}\right)-\epsilon\left(x^{\prime \prime \prime \prime}-X^{\prime \prime \prime}\right) \epsilon\left(y-X^{\prime \prime \prime}\right)\right] \\
& -\frac{i}{2} K \theta\left(Y-X^{\prime \prime \prime}\right) \int_{-X^{\prime \prime \prime \prime}}^{X^{\prime \prime \prime \prime}} d x^{\prime \prime \prime \prime} \int_{-Y^{\prime}}^{Y^{\prime}} d y^{\prime} j_{x}^{L,+}\left(x^{\prime \prime \prime \prime}\right) j_{t}^{L,-}\left(y^{\prime}\right) \\
& \times\left[\epsilon\left(x^{\prime \prime \prime \prime}+X^{\prime \prime \prime}\right) \epsilon\left(y^{\prime}+X^{\prime \prime \prime}\right)-\epsilon\left(x^{\prime \prime \prime \prime}-X^{\prime \prime \prime}\right) \epsilon\left(y^{\prime}-X^{\prime \prime \prime}\right)\right] \\
& +\frac{i}{4} \frac{(1+C)^{2}+K^{2}}{1+C} \theta\left(Y^{\prime \prime}-X^{\prime}\right) \int_{-X}^{X} d x \int_{-X^{\prime \prime}}^{X^{\prime \prime}} d x^{\prime \prime} j_{t}^{L,+}(x) j_{t}^{L,-}\left(x^{\prime \prime}\right) \\
& \times \epsilon\left(x-x^{\prime \prime}\right)\left[\epsilon\left(x+X^{\prime}\right)-\epsilon\left(x-X^{\prime}\right)\right] \\
& +\frac{i}{4} \frac{(1+C)^{2}+K^{2}}{1+C} \theta\left(Y^{\prime \prime}-X^{\prime}\right) \int_{-X}^{X} d x \int_{-X^{\prime \prime}}^{X^{\prime \prime}} d x^{\prime \prime} j_{t}^{L,-}(x) j_{t}^{L,+}\left(x^{\prime \prime}\right) \\
& \times \epsilon\left(x-x^{\prime \prime}\right)\left[\epsilon\left(x+X^{\prime}\right)-\epsilon\left(x-X^{\prime}\right)\right] \\
& +\frac{i}{4} \frac{(1+C)^{2}+K^{2}}{1+C} \theta\left(Y^{\prime \prime}-X\right) \int_{-X^{\prime}}^{X^{\prime}} d x^{\prime} \int_{-X^{\prime \prime}}^{X^{\prime \prime}} d x^{\prime \prime} j_{t}^{L, 3}\left(x^{\prime}\right) j_{t}^{L, 3}\left(x^{\prime \prime}\right) \\
& \times\left[\epsilon\left(x^{\prime}+X\right) \epsilon\left(x^{\prime \prime}+X\right)-\epsilon\left(x^{\prime}-X\right) \epsilon\left(x^{\prime \prime}-X\right)\right] \\
& +\frac{i}{4} \frac{(1+C)^{2}+K^{2}}{1+C} \theta\left(Y^{\prime \prime}-X^{\prime}\right) \int_{-X}^{X} d x \int_{-X^{\prime \prime}}^{X^{\prime \prime}} d x^{\prime \prime} j_{t}^{L, 3}(x) j_{t}^{L, 3}\left(x^{\prime \prime}\right) \\
& \times \epsilon\left(x-x^{\prime \prime}\right)\left[\epsilon\left(x+X^{\prime}\right)-\epsilon\left(x-X^{\prime}\right)\right] \\
& +\frac{i}{4} \frac{(1+C)^{2}+K^{2}}{1+C} \theta\left(Y^{\prime \prime}-X^{\prime \prime}\right) \int_{-X}^{X} d x \int_{-X^{\prime}}^{X^{\prime}} d x^{\prime} j_{t}^{L, 3}(x) j_{t}^{L, 3}\left(x^{\prime}\right) \\
& \times \epsilon\left(x-x^{\prime}\right)\left[\epsilon\left(x+X^{\prime \prime}\right)-\epsilon\left(x-X^{\prime \prime}\right)\right]
\end{aligned}
$$

Then, by taking the limits in which $X, X^{\prime}, X^{\prime \prime}, X^{\prime \prime \prime}, X^{\prime \prime \prime \prime}$ and $X^{\prime \prime \prime \prime \prime \prime}$ are sent to infinity, the expression is simplified as

$$
\begin{aligned}
& \left\{Q_{(1)}^{L, 3}\left(Y, Y^{\prime}, Y^{\prime \prime}\right),\left\{Q_{(1)}^{L,+}, Q_{(1)}^{L,-}\right\}_{\mathrm{P}}\right\}_{\mathrm{P}} \\
= & \frac{K}{2} \int_{-Y}^{Y} d x \int_{-\infty}^{\infty} d x^{\prime} \int_{-Y^{\prime}}^{Y^{\prime}} d y^{\prime} \epsilon\left(x-x^{\prime}\right) \epsilon\left(x-y^{\prime}\right) j_{t}^{L,+}(x) j_{t}^{L, 3}\left(x^{\prime}\right) j_{t}^{L,-}\left(y^{\prime}\right) \\
& -\frac{K}{2} \int_{-Y^{\prime}}^{Y^{\prime}} d x \int_{-\infty}^{\infty} d x^{\prime} \int_{-Y}^{Y} d y \epsilon\left(x-x^{\prime}\right) \epsilon(x-y) j_{t}^{L,-}(x) j_{t}^{L, 3}\left(x^{\prime}\right) j_{t}^{L,+}(y)
\end{aligned}
$$




$$
\begin{aligned}
& +\frac{K}{2} \int_{-Y^{\prime}}^{Y^{\prime}} d x^{\prime} \int_{-\infty}^{\infty} d x^{\prime \prime} \int_{-Y}^{Y} d y \epsilon\left(x^{\prime}-y\right) \epsilon\left(x^{\prime}-x^{\prime \prime}\right) j_{t}^{L, 3}\left(x^{\prime}\right) j_{t}^{L,-}\left(x^{\prime \prime}\right) j_{t}^{L,+}(y) \\
& +\frac{K}{2} \int_{-\infty}^{\infty} d x^{\prime} \int_{-Y^{\prime}}^{Y^{\prime}} d x^{\prime \prime} \int_{-Y}^{Y} d y \epsilon\left(x^{\prime \prime}-y\right) \epsilon\left(x^{\prime \prime}-x^{\prime}\right) j_{t}^{L,-}\left(x^{\prime \prime}\right) j_{t}^{L, 3}\left(x^{\prime}\right) j_{t}^{L,+}(y) \\
& -\frac{K}{2} \int_{-Y}^{Y} d x^{\prime} \int_{-\infty}^{\infty} d x^{\prime \prime} \int_{-Y^{\prime}}^{Y^{\prime}} d y^{\prime} \epsilon\left(x^{\prime}-y^{\prime}\right) \epsilon\left(x^{\prime}-x^{\prime \prime}\right) j_{t}^{L, 3}\left(x^{\prime}\right) j_{t}^{L,+}\left(x^{\prime \prime}\right) j_{t}^{L,-}\left(y^{\prime}\right) \\
& -\frac{K}{2} \int_{-\infty}^{\infty} d x^{\prime} \int_{-Y}^{Y} d x^{\prime \prime} \int_{-Y^{\prime}}^{Y^{\prime}} d y^{\prime} \epsilon\left(x^{\prime \prime}-y^{\prime}\right) \epsilon\left(x^{\prime \prime}-x^{\prime}\right) j_{t}^{L,+}\left(x^{\prime \prime}\right) j_{t}^{L, 3}\left(x^{\prime}\right) j_{t}^{L,-}\left(y^{\prime}\right) \\
& +\frac{i}{2} \frac{(1+C)^{2}+K^{2}}{1+C} \int_{-Y}^{Y} d x^{\prime \prime \prime} \int_{-Y^{\prime}}^{Y^{\prime}} d y^{\prime} \epsilon\left(x^{\prime \prime \prime}-y^{\prime}\right) j_{t}^{L,+}\left(x^{\prime \prime \prime}\right) j_{t}^{L,-}\left(y^{\prime}\right) \\
& -\frac{i}{2} \frac{(1+C)^{2}+K^{2}}{1+C} \int_{-Y^{\prime}}^{Y^{\prime}} d x^{\prime \prime \prime} \int_{-Y}^{Y} d y \epsilon\left(x^{\prime \prime \prime}-y\right) j_{t}^{L,+}(y) j_{t}^{L,-}\left(x^{\prime \prime \prime}\right) \\
& +i K \int_{-Y^{\prime}}^{Y^{\prime}} d x^{\prime \prime \prime \prime} \int_{-Y}^{Y} d y \epsilon\left(y-x^{\prime \prime \prime \prime \prime}\right) j_{t}^{L,+}(y) j_{x}^{L,-}\left(x^{\prime \prime \prime \prime}\right) \\
& -i K \int_{-Y}^{Y} d x^{\prime \prime \prime \prime} \int_{-Y^{\prime}}^{Y^{\prime}} d y^{\prime} \epsilon\left(x^{\prime \prime \prime \prime}-y^{\prime}\right) j_{x}^{L,+}\left(x^{\prime \prime \prime \prime}\right) j_{t}^{L,-}\left(y^{\prime}\right) \\
& -\frac{i}{2} \frac{(1+C)^{2}+K^{2}}{1+C} \int_{-Y^{\prime \prime}}^{Y^{\prime \prime}} d x \int_{-\infty}^{\infty} d x^{\prime \prime} \epsilon\left(x-x^{\prime \prime}\right) j_{t}^{L,+}(x) j_{t}^{L,-}\left(x^{\prime \prime}\right) \\
& -\frac{i}{2} \frac{(1+C)^{2}+K^{2}}{1+C} \int_{-Y^{\prime \prime}}^{Y^{\prime \prime}} d x \int_{-\infty}^{\infty} d x^{\prime \prime} \epsilon\left(x-x^{\prime \prime}\right) j_{t}^{L,-}(x) j_{t}^{L,+}\left(x^{\prime \prime}\right) \\
& +\frac{i}{2} \frac{(1+C)^{2}+K^{2}}{1+C} \int_{-Y^{\prime \prime}}^{Y^{\prime \prime}} d x^{\prime} \int_{-\infty}^{\infty} d x^{\prime \prime} \epsilon\left(x^{\prime}-x^{\prime \prime}\right) j_{t}^{L, 3}\left(x^{\prime}\right) j_{t}^{L, 3}\left(x^{\prime \prime}\right) \\
& +\frac{i}{2} \frac{(1+C)^{2}+K^{2}}{1+C} \int_{-\infty}^{\infty} d x^{\prime} \int_{-Y^{\prime \prime}}^{Y^{\prime \prime}} d x^{\prime \prime} \epsilon\left(x^{\prime \prime}-x^{\prime}\right) j_{t}^{L, 3}\left(x^{\prime \prime}\right) j_{t}^{L, 3}\left(x^{\prime}\right) \\
& -\frac{i}{2} \frac{(1+C)^{2}+K^{2}}{1+C} \int_{-Y^{\prime \prime}}^{Y^{\prime \prime}} d x \int_{-\infty}^{\infty} d x^{\prime \prime} \epsilon\left(x-x^{\prime \prime}\right) j_{t}^{L, 3}(x) j_{t}^{L, 3}\left(x^{\prime \prime}\right) \\
& -\frac{i}{2} \frac{(1+C)^{2}+K^{2}}{1+C} \int_{-Y^{\prime \prime}}^{Y^{\prime \prime}} d x \int_{-\infty}^{\infty} d x^{\prime} \epsilon\left(x-x^{\prime}\right) j_{t}^{L, 3}(x) j_{t}^{L, 3}\left(x^{\prime}\right) \\
& + \text { (no ambiguity). }
\end{aligned}
$$

Finally, by sending $Y, Y^{\prime}$ and $Y^{\prime \prime}$ to infinity, the ambiguous terms are written as

$$
\begin{aligned}
& \left\{Q_{(1)}^{L, 3},\left\{Q_{(1)}^{L,+}, Q_{(1)}^{L,-}\right\}_{\mathrm{P}}\right\}_{\mathrm{P}} \\
= & i K \int_{-\infty}^{\infty} d x \int_{-\infty}^{\infty} d y \epsilon(x-y)\left[j_{t}^{L,+}(x) j^{L,-} x(y)-j_{x}^{L,+}(x) j_{t}^{L,-}(y)\right]+(\text { no ambiguity })
\end{aligned}
$$

The unambiguous terms are evaluated as

$$
\frac{1}{4} Q_{(0)}^{L, 3}\left[Q_{(0)}^{L,+} Q_{(1)}^{L,-}-Q_{(0)}^{L,-} Q_{(1)}^{L,+}\right]
$$




$$
-i K \int_{-\infty}^{\infty} d x \int_{-\infty}^{\infty} d y \epsilon(x-y)\left[j_{t}^{L,+}(x) j^{L,-} x(y)-j_{x}^{L,+}(x) j_{t}^{L,-}(y)\right] .
$$

Thus the relation (B.14) has been shown, though the expression was quite messy in the middle of the computation. The other Serre relations can also be shown in a similar way.

\section{B.2 q-deformed $s u(2)_{\mathrm{R}}$}

The Poisson brackets of $Q^{R, 3}$ and $Q^{R, \pm}$ are next computed.

The level 0 charge $Q^{R, 3}$ and the non-local field $\chi(x)$ are regularized as, respectively,

$$
\begin{aligned}
& Q^{R, 3}\left(X_{0}, X_{1}\right)=\int_{-X_{0}}^{X_{1}} d x j_{t}^{R, 3}(x), \\
& \chi\left(x ; X_{0}, X_{1}\right)=-\frac{1}{2} \int_{-X_{0}}^{X_{1}} d x^{\prime} \epsilon\left(x-x^{\prime}\right) j_{t}^{R, 3}\left(x^{\prime}\right), \quad x \in\left[-X_{0}, X_{1}\right] .
\end{aligned}
$$

Note that for $\chi\left(x ; X_{0}, X_{1}\right)$ the domain of $x$ is restricted. The restriction prevents us from taking the $x \rightarrow \pm \infty$ limit before $-X_{0}$ and $X_{1}$ are sent to infinity. Hence the relation

$$
\lim _{x \rightarrow \pm \infty} \chi\left(x ; X_{0}, X_{1}\right)=\mp \frac{1}{2} Q^{R, 3}\left(X_{0}, X_{1}\right)
$$

is not valid any more. The Poisson brackets of the regularized charges $Q^{R, 3}$ and $\chi(x)$ are

$$
\begin{aligned}
& \left\{Q^{R, 3}\left(X_{0}, X_{1}\right), Q^{R, 3}\left(Y_{0}, Y_{1}\right)\right\}_{\mathrm{P}}=2 K\left[\theta\left(X_{0}-Y_{0}\right)-\theta\left(X_{1}-Y_{1}\right)\right], \\
& \left\{\chi\left(x ; X_{0}, X_{1}\right), Q^{R, 3}\left(Y_{0}, Y_{1}\right)\right\}_{\mathrm{P}}=-K\left[2 \theta\left(x-Y_{1}\right)-2 \theta\left(x+Y_{0}\right)\right. \\
& \left.+\theta\left(Y_{0}-X_{0}\right)+\theta\left(Y_{1}-X_{1}\right)\right] \\
& \left\{\chi\left(x ; X_{0}, X_{1}\right), \chi\left(y ; Y_{0}, Y_{1}\right)\right\}_{\mathrm{P}}=K\left[-\epsilon(x-y)+\theta\left(x-Y_{1}\right)+\theta\left(x+Y_{0}\right)-\theta\left(y-X_{1}\right)\right. \\
& \left.-\theta\left(y+X_{0}\right)+\frac{1}{2} \theta\left(X_{0}-Y_{0}\right)-\frac{1}{2} \theta\left(X_{1}-Y_{1}\right)\right] .
\end{aligned}
$$

Next we define the regularized level 1 charges as

$$
Q^{R, \pm}\left(X_{0}, X_{1} ; X_{0}^{\prime}, X_{1}^{\prime}\right)=\int_{-X_{0}}^{X_{1}} d x \mathrm{e}^{\gamma^{ \pm} \chi\left(x ; X_{0}^{\prime}, X_{1}^{\prime}\right)} i_{t}^{R, \pm}(x), \quad\left[-X_{0}, X_{1}\right] \subset\left[-X_{0}^{\prime}, X_{1}^{\prime}\right]
$$

Note that the interval $\left[-X_{0}, X_{1}\right]$ is included in the interval $\left[-X_{0}^{\prime}, X_{1}^{\prime}\right]$. Then the Poisson brackets of the regularized charges $Q^{R, 3}$ and $Q^{R, \pm}$ are given by

$$
\left\{Q^{R, \pm}\left(X_{0}, X_{1} ; X_{0}^{\prime}, X_{1}^{\prime}\right), Q^{R, 3}\left(Y_{0}, Y_{1}\right)\right\}_{\mathrm{P}}
$$




$$
\begin{aligned}
& = \pm i Q^{R, \pm}\left(\min \left(X_{0}, Y_{0}\right), \min \left(X_{1}, Y_{1}\right) ; X_{0}^{\prime}, X_{1}^{\prime}\right) \\
& \pm \frac{i}{2}\left(1-\frac{\gamma^{ \pm}}{\gamma^{\mp}}\right)\left[2 Q^{R, \pm}\left(\min \left(X_{0}, Y_{0}\right), \min \left(X_{1}, Y_{1}\right) ; X_{0}^{\prime}, X_{1}^{\prime}\right)\right. \\
& \left.-\left[\theta\left(Y_{0}-X_{0}^{\prime}\right)+\theta\left(Y_{1}-X_{1}^{\prime}\right)\right] Q^{R, \pm}\left(X_{0}, X_{1} ; X_{0}^{\prime}, X_{1}^{\prime}\right)\right], \\
& \left\{Q^{R,+}\left(X_{0}, X_{1} ; X_{0}^{\prime}, X_{1}^{\prime}\right), Q^{R,-}\left(Y_{0}, Y_{1} ; Y_{0}^{\prime}, Y_{1}^{\prime}\right)\right\}_{\mathrm{P}} \\
& =i \frac{\gamma^{-}}{\gamma^{+}} \frac{1}{\gamma^{+}+\gamma^{-}}\left[\mathrm{e}^{\gamma^{+} \chi\left(\min \left(X_{1}, Y_{1}\right) ; X_{0}^{\prime}, X_{1}^{\prime}\right)+\gamma^{-} \chi\left(\min \left(X_{1}, Y_{1}\right) ; Y_{0}^{\prime}, Y_{1}^{\prime}\right)}\right. \\
& \left.-\mathrm{e}^{\gamma^{+} \chi\left(-\min \left(X_{0}, Y_{0}\right) ; X_{0}^{\prime}, X_{1}^{\prime}\right)+\gamma^{-} \chi\left(-\min \left(X_{0}, Y_{0}\right) ; Y_{0}^{\prime}, Y_{1}^{\prime}\right)}\right] \\
& -i \frac{\gamma^{+}-\gamma^{-}}{\gamma^{+} \gamma^{-}}\left[\theta\left(X_{0}-Y_{0}\right) \mathrm{e}^{\gamma^{+} \chi\left(-Y_{0} ; X_{0}^{\prime}, X_{1}^{\prime}\right)+\gamma^{-} \chi\left(-Y_{0} ; Y_{0}^{\prime}, Y_{1}^{\prime}\right)}\right. \\
& \left.-\theta\left(X_{1}-Y_{1}\right) \mathrm{e}^{\gamma^{+} \chi\left(Y_{1} ; X_{0}^{\prime}, X_{1}^{\prime}\right)+\gamma^{-} \chi\left(Y_{1} ; Y_{0}^{\prime}, Y_{1}^{\prime}\right)}\right] \\
& -\frac{1}{2}\left(\gamma^{+}-\gamma^{-}\right) Q^{R,-}\left(Y_{0}, Y_{1} ; Y_{0}^{\prime}, Y_{1}^{\prime}\right) \\
& \times \int_{-X_{0}}^{X_{1}} d x\left[\theta\left(x-Y_{1}^{\prime}\right)+\theta\left(x+Y_{0}^{\prime}\right)\right] \mathrm{e}^{\gamma^{+} \chi\left(x ; X_{0}^{\prime}, X_{1}^{\prime}\right)} i_{t}^{R,+}(x) \\
& +\frac{1}{2}\left(\gamma^{+}-\gamma^{-}\right) Q^{R,+}\left(X_{0}, X_{1} ; X_{0}^{\prime}, X_{1}^{\prime}\right) \\
& \times \int_{-Y_{0}}^{Y_{1}} d y\left[\theta\left(y-X_{1}^{\prime}\right)+\theta\left(y+X_{0}^{\prime}\right)\right] \mathrm{e}^{\gamma^{-} \chi\left(y ; Y_{0}^{\prime}, Y_{1}^{\prime}\right)} i_{t}^{R,-}(y) \\
& -\frac{1}{4}\left(\gamma^{+}-\gamma^{-}\right)\left[\theta\left(X_{0}^{\prime}-Y_{0}^{\prime}\right)-\theta\left(X_{1}^{\prime}-Y_{1}^{\prime}\right)\right] \\
& \times Q^{R,+}\left(X_{0}, X_{1} ; X_{0}^{\prime}, X_{1}^{\prime}\right) Q^{R,-}\left(Y_{0}, Y_{1} ; Y_{0}^{\prime}, Y_{1}^{\prime}\right) \text {. }
\end{aligned}
$$

\section{The order of limits}

Let us consider the order of limits in the Poisson brackets computed so far.

The first is the Poisson brackets in (B.22), which depend on the order of limits. Note that

the bracket $\left\{Q^{R, 3}, Q^{R, 3}\right\}_{\mathrm{P}}$ should vanish. A prescription is to take a specific regularization as follows:

$$
Q^{R, 3}(X)=Q^{R, 3}(X, X)
$$

Then the Poisson bracket vanishes properly,

$$
\left\{Q^{R, 3}(X), Q^{R, 3}(Y)\right\}_{\mathrm{P}}=0
$$

Thus, after taking the limits, the desired result is obtained as

$$
\left\{Q^{R, 3}, Q^{R, 3}\right\}_{\mathrm{P}}=0
$$


Similarly, the level 1 charges can be regularized as

$$
Q^{R, \pm}\left(X ; X^{\prime}\right)=Q^{R, \pm}\left(X, X ; X^{\prime}, X^{\prime}\right) .
$$

The Poisson brackets of $Q^{R, \pm}\left(X, X^{\prime}\right)$ and $Q^{R, 3}(Y)$ are

$$
\begin{aligned}
&\left\{Q^{R, \pm}\left(X ; X^{\prime}\right), Q^{R, 3}(Y)\right\}_{\mathrm{P}}= \pm i Q^{R, \pm}\left(\min (X, Y) ; X^{\prime}\right) \\
& \pm i\left(1-\frac{\gamma^{ \pm}}{\gamma^{\mp}}\right)\left[Q^{R, \pm}\left(\min (X, Y) ; X^{\prime}\right)-\theta\left(Y-X^{\prime}\right) Q^{R, \pm}\left(X ; X^{\prime}\right)\right]
\end{aligned}
$$

and the Poisson bracket between $Q^{R, \pm}\left(X, X^{\prime}\right)$ is

$$
\begin{aligned}
& \left\{Q^{R,+}\left(X ; X^{\prime}\right), Q^{R,-}\left(Y ; Y^{\prime}\right)\right\}_{\mathrm{P}} \\
= & i \frac{\gamma^{-}}{\gamma^{+}} \frac{1}{\gamma^{+}+\gamma^{-}}\left[\mathrm{e}^{\gamma^{+} \chi\left(\min (X, Y) ; X^{\prime}\right)+\gamma^{-} \chi\left(\min (X, Y) ; Y^{\prime}\right)}-\mathrm{e}^{\gamma^{+} \chi\left(-\min (X, Y) ; X^{\prime}\right)+\gamma^{-} \chi\left(-\min (X, Y) ; Y^{\prime}\right)}\right] \\
& -i \frac{\gamma^{+}-\gamma^{-}}{\gamma^{+} \gamma^{-}} \theta(X-Y)\left[\mathrm{e}^{\gamma^{+} \chi\left(-Y ; X^{\prime}\right)+\gamma^{-} \chi\left(-Y ; Y^{\prime}\right)}-\mathrm{e}^{\gamma^{+} \chi\left(Y ; X^{\prime}\right)+\gamma^{-} \chi\left(Y ; Y^{\prime}\right)}\right] \\
& -\frac{1}{2}\left(\gamma^{+}-\gamma^{-}\right) Q^{R,-}\left(Y ; Y^{\prime}\right) \int_{-X}^{X} d x\left[\theta\left(x-Y^{\prime}\right)+\theta\left(x+Y^{\prime}\right)\right] \mathrm{e}^{\gamma^{+} \chi\left(x ; X^{\prime}\right)} i_{t}^{R,+}(x) \\
& +\frac{1}{2}\left(\gamma^{+}-\gamma^{-}\right) Q^{R,+}\left(X ; X^{\prime}\right) \int_{-Y}^{Y} d y\left[\theta\left(y-X^{\prime}\right)+\theta\left(y+X^{\prime}\right)\right] \mathrm{e}^{\gamma^{-} \chi\left(y ; Y^{\prime}\right)} i_{t}^{R,-}(y),
\end{aligned}
$$

where $\chi(x ; X)$ is defined as

$$
\chi(x ; X) \equiv \chi(x ; X, X) .
$$

Thus the Poisson brackets in (B.28) and (B.29) depend on the order of limits. The appearance of the order of limits is due to the presence of the Wess-Zumino term. When $K=0$, $\gamma_{+}=\gamma_{-}$and no ambiguity exists, as discussed in [25].

Let us consider the order of limits for the Poisson bracket (B.28) . A prescription is to take the limit $Y \rightarrow \infty$ before the limit $X^{\prime} \rightarrow \infty$ :

$$
\left\{Q^{R, \pm}\left(X ; X^{\prime}\right), Q^{R, 3}\right\}_{\mathrm{P}}= \pm i Q^{R, \pm}\left(X ; X^{\prime}\right) .
$$

Then one can obtain the desired expression,

$$
\left\{Q^{R, \pm}, Q^{R, 3}\right\}_{\mathrm{P}}= \pm i Q^{R, \pm},
$$

by sending $X$ and $X^{\prime}$ to infinity. 
The next is the order of limits in (B.29) . A prescription is to take the $X^{\prime} \rightarrow \infty$ limit before the $X \rightarrow \infty$ limit. Thus the regularized charge is introduced as

$$
Q^{R, \pm}(X)=\lim _{X^{\prime} \rightarrow \infty} Q^{R, \pm}\left(X ; X^{\prime}\right)
$$

The Poisson bracket between $Q^{R, \pm}$ is

$$
\begin{aligned}
\left\{Q^{R,+}(X), Q^{R,-}(Y)\right\}_{\mathrm{P}}= & i \frac{\gamma^{-}}{\gamma^{+}} \frac{1}{\gamma^{+}+\gamma^{-}}\left[\mathrm{e}^{\left(\gamma^{+}+\gamma^{-}\right) \chi(\min (X, Y))}-\mathrm{e}^{\left(\gamma^{+}+\gamma^{-}\right) \chi(-\min (X, Y))}\right] \\
& -i \frac{\gamma^{+}-\gamma^{-}}{\gamma^{+} \gamma^{-}} \theta(X-Y)\left[\mathrm{e}^{\left(\gamma^{+}+\gamma^{-}\right) \chi(-Y)}-\mathrm{e}^{\left(\gamma^{+}+\gamma^{-}\right) \chi(Y)}\right] .
\end{aligned}
$$

The second term depends on the order of limits. This ambiguity appears as only the overall factor and hence it can be absorbed by rescaling the charges. Here the order of limits is fixed in the following way:

$$
\begin{aligned}
\left\{Q^{R,+}, Q^{R,-}\right\}_{\mathrm{P}} & \equiv \lim _{X \rightarrow \infty}\left\{Q^{R,+}(X), Q^{R,-}(X)\right\}_{\mathrm{P}} \\
& =\frac{i}{2} \frac{\left(\gamma^{+}\right)^{2}+\left(\gamma^{-}\right)^{2}}{\gamma^{+} \gamma^{-}\left(\gamma^{+}+\gamma^{-}\right)} \lim _{X \rightarrow \infty}\left[\mathrm{e}^{\left(\gamma^{+}+\gamma^{-}\right) \chi(X)}-\mathrm{e}^{\left(\gamma^{+}+\gamma^{-}\right) \chi(-X)}\right] \\
& =-i \frac{\left(\gamma^{+}\right)^{2}+\left(\gamma^{-}\right)^{2}}{2 \gamma^{+} \gamma^{-}} \frac{2}{\left(\gamma^{+}+\gamma^{-}\right)} \sinh \left[\frac{\left(\gamma^{+}+\gamma^{-}\right)}{2} Q^{R, 3}\right]
\end{aligned}
$$

Thus the q-deformed su(2) algebra has been shown with some prescriptions for the order of limits, where the $q$-parameter is defined as

$$
q \equiv \exp \left[\frac{\gamma_{+}+\gamma_{-}}{2}\right] .
$$

\section{B.3 A deformation of quantum affine algebra}

Let us here present how to compute the deformed quantum affine algebra presented in Sec.4.4. In the middle of the computation, again, it is necessary to introduce some prescriptions to treat the ambiguities coming from the order of limits.

The Poisson bracket between $Q_{(1)}^{R,-}$ and $\widetilde{Q}_{(1)}^{R,+}$ is first computed. For the regularized charges, it is given by

$$
\begin{aligned}
& \left\{Q_{(1)}^{R,-}(X), \widetilde{Q}_{(1)}^{R,+}(Y)\right\}_{\mathrm{P}} \\
= & \frac{1}{2} \mathrm{e}^{-2 \beta} \sinh 2 \alpha\left[\int_{-X}^{X} d x \int_{-Y}^{Y} d y \epsilon(x-y) j_{t}^{R,-}(x) \widetilde{j}_{t}^{R,+}(y)\right.
\end{aligned}
$$




$$
\begin{gathered}
\left.-2 i \mathrm{e}^{2 \beta} \int_{-\min (X, Y)}^{\min (X, Y)} d x j_{x}^{R, 3}(x)+2 i \mathrm{e}^{2 \beta} \frac{\cosh 2 \alpha}{\sinh 2 \alpha} Q_{(0)}^{R, 3}(\min (X, Y))\right] \\
=\frac{1}{2} \mathrm{e}^{-2 \beta} \sinh 2 \alpha \bar{Q}_{(2)}^{R, 3}(X, Y, \min (X, Y)) .
\end{gathered}
$$

Here the level 2 charge $\bar{Q}_{(2)}^{R, 3}$ is regularized as

$$
\begin{aligned}
\bar{Q}_{(2)}^{R, 3}\left(X, X^{\prime}, X^{\prime \prime}\right)= & \int_{-X}^{X} d x \int_{-X^{\prime}}^{X^{\prime}} d y \epsilon(x-y) j_{t}^{R,-}(x) \widetilde{j}_{t}^{R,+}(y) \\
& -2 i \mathrm{e}^{2 \beta} \int_{-X^{\prime \prime}}^{X^{\prime \prime}} d x j_{x}^{R, 3}(x)+2 i \mathrm{e}^{2 \beta} \frac{\cosh 2 \alpha}{\sinh 2 \alpha} Q_{(0)}^{R, 3}\left(X^{\prime \prime}\right) .
\end{aligned}
$$

Note that no ambiguous term is contained in this Poisson bracket (B.35) . Thus, by taking the $X, Y \rightarrow \infty$ limits, we obtain the following:

$$
\left\{Q_{(1)}^{R,-}, \widetilde{Q}_{(1)}^{R,+}\right\}_{\mathrm{P}}=\frac{1}{2} \mathrm{e}^{-2 \beta} \sinh 2 \alpha \bar{Q}_{(2)}^{R, 3} .
$$

As a next step, the Poisson bracket between $\bar{Q}_{(2)}^{R, 3}$ and $Q_{(1)}^{R,-}$ is computed. For the regularized charges, it is evaluated as

$$
\begin{aligned}
& \left\{\bar{Q}_{(2)}^{R, 3}\left(X, X^{\prime}, X^{\prime \prime}\right), Q_{(1)}^{R,-}(Y)\right\}_{\mathrm{P}} \\
= & -\frac{1}{2} \mathrm{e}^{-2 \beta} \sinh 2 \alpha \\
& \times\left[\int_{-X}^{X} d x \int_{-X^{\prime}}^{X^{\prime}} d x^{\prime} \int_{-Y}^{Y} d y \epsilon\left(x-x^{\prime}\right)\left[\epsilon(x-y)-\epsilon\left(x^{\prime}-y\right)\right] j_{t}^{R,-}(x) \widetilde{j}_{t}^{R,+}\left(x^{\prime}\right) j_{t}^{R,-}(y)\right. \\
& \quad-2 i \mathrm{e}^{2 \beta} \int_{-X}^{X} d x \int_{-\min \left(X^{\prime}, Y\right)}^{\min \left(X^{\prime}, Y\right)} d x^{\prime} \epsilon\left(x-x^{\prime}\right) j_{t}^{R,-}(x)\left(j_{x}^{R, 3}\left(x^{\prime}\right)-\frac{\cosh 2 \alpha}{\sinh 2 \alpha} j_{t}^{R, 3}\left(x^{\prime}\right)\right) \\
& \left.\quad-\frac{4 \mathrm{e}^{2 \beta}}{\sinh 2 \alpha} \int_{-\min \left(X^{\prime \prime}, Y\right)}^{\min \left(X^{\prime \prime}, Y\right)} d x^{\prime \prime} j_{x}^{R,-}\left(x^{\prime \prime}\right)+\frac{4 \mathrm{e}^{2 \beta} \cosh 2 \alpha}{\sinh ^{2} 2 \alpha} Q_{(1)}^{R,-}\left(\min \left(X^{\prime \prime}, Y\right)\right)\right] \\
= & -\frac{1}{2} \mathrm{e}^{-2 \beta} \sinh 2 \alpha \\
& \times\left[Q_{(3)}^{R,-}\left(X, X^{\prime}, Y, X, \min \left(X^{\prime}, Y\right), \min \left(X^{\prime \prime}, Y\right)\right)+\frac{2}{3} Q_{(1)}^{R,-}(X)(\min (X, Y))-\theta\left(Y-X^{\prime}\right) Q_{(1)}^{R,-}(X)\right\} \\
& +\frac{1}{2} \int_{-X}^{X,+} d x \int_{-X^{\prime}}^{X^{\prime}} d x^{\prime} \int_{-Y}^{Y} d y \epsilon(x-y)\left[\epsilon\left(x-x_{(1)}^{R,-}(Y)+\epsilon\left(y-x^{\prime}\right)\right] j_{t}^{R,-}(x) \widetilde{j}_{t}^{R,+}\left(x^{\prime}\right) j_{t}^{R,-}\left(x^{\prime \prime}\right)\right] \\
& +4(K-i A)\left\{Q_{(1)}^{R,-}(\min (X, Y))-\theta\left(Y-X^{\prime}\right) Q_{(1)}^{R,-}(X)\right\} .
\end{aligned}
$$


Here the last term

$$
4(K-i A)\left\{Q_{(1)}^{R,-}(\min (X, Y))-\theta\left(Y-X^{\prime}\right) Q_{(1)}^{R,-}(X)\right\}
$$

depends on the order of limits. Note that the level 3 charge $Q_{(3)}^{R,-}$ is regularized as

$$
\begin{aligned}
& Q_{(3)}^{R,-}\left(X, X^{\prime}, X^{\prime \prime}, X^{\prime \prime \prime}, X^{\prime \prime \prime \prime}, X^{\prime \prime \prime \prime \prime}\right) \\
& =\frac{1}{2} \int_{-X}^{X} d x \int_{-X^{\prime}}^{X^{\prime}} d x^{\prime} \int_{-X^{\prime \prime}}^{X^{\prime \prime}} d x^{\prime \prime} \epsilon\left(x^{\prime}-x\right) \epsilon\left(x^{\prime}-x^{\prime \prime}\right) j_{t}^{R,-}(x) \widetilde{j}_{t}^{R,+}\left(x^{\prime}\right) j_{t}^{R,-}\left(x^{\prime \prime}\right) \\
& -2 i \mathrm{e}^{2 \beta} \int_{-X^{\prime \prime \prime}}^{X^{\prime \prime \prime}} d x^{\prime \prime \prime} \int_{-X^{\prime \prime \prime \prime}}^{X^{\prime \prime \prime \prime}} d x^{\prime \prime \prime \prime} \epsilon\left(x^{\prime \prime \prime}-x^{\prime \prime \prime \prime}\right) j_{t}^{R,-}\left(x^{\prime \prime \prime}\right)\left(j_{x}^{R, 3}\left(x^{\prime \prime \prime \prime}\right)-\frac{\cosh 2 \alpha}{\sinh 2 \alpha} j_{t}^{R, 3}\left(x^{\prime \prime \prime \prime}\right)\right) \\
& -\frac{4 \mathrm{e}^{2 \beta}}{\sinh 2 \alpha} \int_{-X^{\prime \prime \prime \prime \prime}}^{X^{\prime \prime \prime \prime \prime}} d x^{\prime \prime \prime \prime \prime \prime \prime} j_{x}^{R,-}\left(x^{\prime \prime \prime \prime \prime \prime}\right)+\frac{4 \mathrm{e}^{2 \beta} \cosh 2 \alpha}{\sinh ^{2} 2 \alpha} Q_{(1)}^{R,-}\left(X^{\prime \prime \prime \prime \prime \prime}\right) \\
& -\frac{1}{6} Q_{(1)}^{R,-}(X) \widetilde{Q}_{(1)}^{R,+}\left(X^{\prime}\right) Q_{(1)}^{R,-}\left(X^{\prime \prime}\right) .
\end{aligned}
$$

A prescription is to take the $Y \rightarrow \infty$ limit before $X$ is sent to infinity so that the ambiguous term vanishes:

$$
\begin{aligned}
& \left\{\bar{Q}_{(2)}^{R, 3}\left(X, X^{\prime}, X^{\prime \prime}\right), Q_{(1)}^{R,-}\right\}_{\mathrm{P}} \\
= & \lim _{Y \rightarrow \infty}\left\{\bar{Q}_{(2)}^{R, 3}\left(X, X^{\prime}, X^{\prime \prime}\right), Q^{R,-}(Y)\right\}_{\mathrm{P}} \\
= & -\frac{1}{2} \mathrm{e}^{-2 \beta} \sinh 2 \alpha\left[Q_{(3)}^{R,-}\left(X, X^{\prime}, \infty, X, X^{\prime}, X^{\prime \prime}\right)+\frac{2}{3} Q_{(1)}^{R,-}(X) \widetilde{Q}_{(1)}^{R,+}\left(X^{\prime}\right) Q_{(1)}^{R,-}\right] \\
& -\frac{1}{4} \mathrm{e}^{-2 \beta} \sinh 2 \alpha \int_{-X}^{X} d x \int_{-X^{\prime}}^{X^{\prime}} d x^{\prime} \int_{-\infty}^{\infty} d y \epsilon(x-y)\left[\epsilon\left(x-x^{\prime}\right)+\epsilon\left(y-x^{\prime}\right)\right] \\
& \quad \times j_{t}^{R,-}(x) \widetilde{j}_{t}^{R,+}\left(x^{\prime}\right) j_{t}^{R,-}(y) .
\end{aligned}
$$

By taking the remaining limits, we obtain the following result:

$$
\left\{\bar{Q}_{(2)}^{R, 3}, Q_{(1)}^{R,-}\right\}_{\mathrm{P}}=-\frac{1}{2} \mathrm{e}^{-2 \beta} \sinh 2 \alpha\left[Q_{(3)}^{R,-}+\frac{2}{3} \widetilde{Q}_{(1)}^{R,+}\left(Q_{(1)}^{R,-}\right)^{2}\right] .
$$

It is interesting to see a higher-level Poisson bracket, for example, the Poisson bracket between $Q_{(3)}^{R,-}$ and $Q_{(1)}^{R,-}$. For the regularized charges, it is given by

$$
\begin{aligned}
& \left\{Q_{(3)}^{R,-}\left(X, X^{\prime}, X^{\prime \prime}, X^{\prime \prime \prime}, X^{\prime \prime \prime \prime}, X^{\prime \prime \prime \prime \prime \prime}\right), Q_{(1)}^{R,-}(Y)\right\}_{\mathrm{P}} \\
= & -\frac{1}{4} \mathrm{e}^{-2 \beta} \sinh 2 \alpha \\
& \times\left[-\frac{1}{2} Q_{(1)}^{R,-}(X) Q_{(1)}^{R,-}(Y) \int_{-X^{\prime}}^{X^{\prime}} d x^{\prime} \int_{-X^{\prime \prime}}^{X^{\prime \prime}} d x^{\prime \prime} \epsilon\left(x^{\prime}-x^{\prime \prime}\right) \widetilde{j}_{t}^{R,+}\left(x^{\prime}\right) j_{t}^{R,-}\left(x^{\prime \prime}\right)\right.
\end{aligned}
$$




$$
\begin{aligned}
& +\frac{1}{2} Q_{(1)}^{R,-}\left(X^{\prime \prime}\right) Q_{(1)}^{R,-}(Y) \int_{-X}^{X} d x \int_{-X^{\prime}}^{X^{\prime}} d x^{\prime} \epsilon\left(x-x^{\prime}\right) j_{t}^{R,-}(x) \widetilde{j}_{t}^{R,+}\left(x^{\prime}\right) \\
& +\frac{1}{2} \int_{-X}^{X} d x \int_{-X^{\prime}}^{X^{\prime}} d x^{\prime} \int_{-X^{\prime \prime}}^{X^{\prime \prime}} d x^{\prime \prime} \int_{-Y}^{Y} d y \\
& \times\left\{\epsilon\left(x^{\prime}-x^{\prime \prime}\right) \epsilon(y-x)\left[\epsilon\left(y-x^{\prime}\right)+\epsilon\left(x-x^{\prime}\right)\right]\right. \\
& \left.+\epsilon\left(x^{\prime}-x\right) \epsilon\left(y-x^{\prime \prime}\right)\left[\epsilon\left(y-x^{\prime}\right)+\epsilon\left(x^{\prime \prime}-x^{\prime}\right)\right]\right\} \\
& \times j_{t}^{R,-}(x) \widetilde{j}_{t}^{R,+}\left(x^{\prime}\right) j_{t}^{R,-}\left(x^{\prime \prime}\right) j_{t}^{R,-}(y) \\
& -2 i \mathrm{e}^{2 \beta} Q_{(1)}^{R,-}(X) Q_{(1)}^{R,-}\left(X^{\prime \prime}\right) \int_{-\min \left(X^{\prime}, Y\right)}^{\min \left(X^{\prime}, Y\right)} d x^{\prime}\left[j_{x}^{R, 3}\left(x^{\prime}\right)-\frac{\cosh 2 \alpha}{\sinh 2 \alpha} j_{t}^{R, 3}\left(x^{\prime}\right)\right] \\
& +4 i \mathrm{e}^{2 \beta} \int_{-X}^{X} d x \int_{-\min \left(X^{\prime}, Y\right)}^{\min \left(X^{\prime}, Y\right)} d x^{\prime} \int_{-X^{\prime \prime}}^{X^{\prime \prime}} d x^{\prime \prime} \epsilon\left(x-x^{\prime}\right) \epsilon\left(x-x^{\prime \prime}\right) \\
& \times j_{t}^{R,-}(x)\left[j_{x}^{R, 3}\left(x^{\prime}\right)-\frac{\cosh 2 \alpha}{\sinh 2 \alpha} j_{t}^{R, 3}\left(x^{\prime}\right)\right] j_{t}^{R,-}\left(x^{\prime \prime}\right) \\
& -4 i \mathrm{e}^{2 \beta} \int_{-X^{\prime \prime \prime}}^{X^{\prime \prime \prime}} d x^{\prime \prime \prime} \int_{-X^{\prime \prime \prime \prime}}^{X^{\prime \prime \prime \prime}} d x^{\prime \prime \prime \prime} \int_{-Y}^{Y} d y \epsilon\left(x^{\prime \prime \prime}-x^{\prime \prime \prime \prime}\right) \epsilon\left(x^{\prime \prime \prime}-y\right) \\
& \times j_{t}^{R,-}\left(x^{\prime \prime \prime}\right)\left[j_{x}^{R, 3}\left(x^{\prime \prime \prime \prime}\right)-\frac{\cosh 2 \alpha}{\sinh 2 \alpha} j_{t}^{R, 3}\left(x^{\prime \prime \prime \prime}\right)\right] j_{t}^{R,-}(y) \\
& -\frac{8 \mathrm{e}^{2 \beta}}{\sinh 2 \alpha} \int_{-X^{\prime \prime \prime}}^{X^{\prime \prime \prime}} d x^{\prime \prime \prime} \int_{-\min \left(X^{\prime \prime \prime \prime}, Y\right)}^{\min \left(X^{\prime \prime \prime \prime}, Y\right)} d x^{\prime \prime \prime \prime \prime} \epsilon\left(x^{\prime \prime \prime}-x^{\prime \prime \prime \prime}\right) j_{t}^{R,-}\left(x^{\prime \prime \prime}\right) j_{x}^{R,-}\left(x^{\prime \prime \prime \prime}\right) \\
& -\frac{8 \mathrm{e}^{2 \beta}}{\sinh 2 \alpha} \int_{-X^{\prime \prime \prime \prime \prime \prime}}^{X^{\prime \prime \prime \prime \prime \prime}} d x^{\prime \prime \prime \prime \prime \prime} \int_{-Y}^{Y} d y \epsilon\left(x^{\prime \prime \prime \prime \prime \prime}-y\right) j_{x}^{R,-}\left(x^{\prime \prime \prime \prime \prime \prime}\right) j_{t}^{R,-}(y) \\
& +\frac{8 \mathrm{e}^{2 \beta} \cosh 2 \alpha}{\sinh ^{2} 2 \alpha} \int_{-X^{\prime \prime \prime}}^{X^{\prime \prime \prime}} d x^{\prime \prime \prime} \int_{-\min \left(X^{\prime \prime \prime \prime}, Y\right)}^{\min \left(X^{\prime \prime \prime \prime}, Y\right)} d x^{\prime \prime \prime \prime \prime} \epsilon\left(x^{\prime \prime \prime}-x^{\prime \prime \prime \prime}\right) \\
& \times j_{t}^{R,-}\left(x^{\prime \prime \prime}\right) j_{t}^{R,-}\left(x^{\prime \prime \prime \prime}\right) \\
& \left.-\frac{1}{3} Q_{(1)}^{R,-}(X) Q_{(1)}^{R,-}\left(X^{\prime \prime}\right) \bar{Q}_{(2)}^{R, 3}\left(Y, X^{\prime}, \min \left(X^{\prime}, Y\right)\right)\right] \\
& +(K-i A) \theta\left(X^{\prime}-Y\right) \\
& \times \int_{-X}^{X} d x \int_{-X^{\prime \prime}}^{X^{\prime \prime}} d x^{\prime \prime}\left[\epsilon(x+Y) \epsilon\left(x^{\prime \prime}+Y\right)-\epsilon(x-Y) \epsilon\left(x^{\prime \prime}-Y\right)\right] j_{t}^{R,-}(x) j_{t}^{R,-}\left(x^{\prime \prime}\right) .
\end{aligned}
$$

Note that the last term

$$
\begin{aligned}
(K-i A) \theta\left(X^{\prime}\right. & -Y) \int_{-X}^{X} d x \int_{-X^{\prime \prime}}^{X^{\prime \prime}} d x^{\prime \prime} \\
& \times\left[\epsilon(x+Y) \epsilon\left(x^{\prime \prime}+Y\right)-\epsilon(x-Y) \epsilon\left(x^{\prime \prime}-Y\right)\right] j_{t}^{R,-}(x) j_{t}^{R,-}\left(x^{\prime \prime}\right) .
\end{aligned}
$$

is proportional to the step function and hence seems likely to be an ambiguous term. However, this is not the case. This term does not depend on the order of limits and it is reduced 
to zero after taking all of the limits. Thus the resulting Poisson bracket is given by

$$
\left\{Q_{(3)}^{R,-}, Q_{(1)}^{R,-}\right\}_{\mathrm{P}}=-\frac{1}{6} \mathrm{e}^{-2 \beta} \sinh 2 \alpha\left(Q_{(1)}^{R,-}\right)^{2} \bar{Q}_{(2)}^{R, 3}
$$

Similarly, the other Poisson brackets are computed, though those are not touched here.

\section{References}

[1] J. M. Maldacena, "The large N limit of superconformal field theories and supergravity," Adv. Theor. Math. Phys. 2 (1998) 231 [Int. J. Theor. Phys. 38 (1999) 1113]. arXiv:hep-th/9711200.

[2] S. S. Gubser, I. R. Klebanov and A. M. Polyakov, "Gauge theory correlators from non-critical string theory," Phys. Lett. B 428 (1998) 105 arXiv:hep-th/9802109.

[3] E. Witten, "Anti-de Sitter space and holography," Adv. Theor. Math. Phys. 2 (1998) 253 arXiv:hep-th/9802150

[4] N. Beisert et al., "Review of AdS/CFT Integrability: An Overview," arXiv:1012.3982 [hep-th].

[5] I. Bena, J. Polchinski and R. Roiban, "Hidden symmetries of the $\operatorname{AdS}_{5} \times \mathrm{S}^{5}$ superstring," Phys. Rev. D 69 (2004) 046002 hep-th/0305116].

[6] M. Lüscher, "Quantum nonlocal charges and absence of particle production in the twodimensional nonlinear sigma model," Nucl. Phys. B 135 (1978) 1.

[7] M. Lüscher and K. Pohlmeyer, "Scattering of massless lumps and nonlocal charges in the two-dimensional classical nonlinear sigma model," Nucl. Phys. B 137 (1978) 46.

[8] E. Brezin, C. Itzykson, J. Zinn-Justin and J. B. Zuber, "Remarks about the existence of nonlocal charges in two-dimensional models," Phys. Lett. B 82 (1979) 442.

[9] D. Bernard, "Hidden Yangians in 2-D massive current algebras," Commun. Math. Phys. 137 (1991) 191.

[10] N. J. MacKay, "On the classical origins of Yangian symmetry in integrable field theory," Phys. Lett. B 281 (1992) 90 [Erratum-ibid. B 308 (1993) 444]. 
[11] E. Abdalla, M. C. Abdalla and K. Rothe, "Non-perturbative methods in twodimensional quantum field theory," World Scientific, 1991.

[12] K. Zarembo, "Strings on Semisymmetric Superspaces," JHEP 1005 (2010) 002 arXiv:1003.0465 [hep-th]].

[13] N. Beisert and P. Koroteev, "Quantum Deformations of the One-Dimensional Hubbard Model," J. Phys. A 41 (2008) 255204 [arXiv:0802.0777 [hep-th]].

[14] N. Beisert, W. Galleas and T. Matsumoto, "A Quantum Affine Algebra for the Deformed Hubbard Chain," J. Phys. A 45 (2012) 365206 [arXiv:1102.5700 [math-ph]].

[15] B. Hoare, T. J. Hollowood and J. L. Miramontes, " $q$-Deformation of the $\mathrm{AdS}_{5} \times \mathrm{S}^{5} \mathrm{Su}$ perstring S-matrix and its Relativistic Limit," JHEP 1203 (2012) 015 arXiv:1112.4485 [hep-th]]; "Bound States of the $q$-Deformed $\mathrm{AdS}_{5} \times \mathrm{S}^{5}$ Superstring S-matrix," JHEP 1210 (2012) 076 arXiv:1206.0010 [hep-th]]; "Restoring Unitarity in the q-Deformed World-Sheet S-Matrix," arXiv:1303.1447 [hep-th].

[16] M. de Leeuw, V. Regelskis and A. Torrielli, "The Quantum Affine Origin of the AdS/CFT Secret Symmetry," J. Phys. A 45 (2012) 175202 [arXiv:1112.4989 [hep-th]].

[17] G. Arutyunov, M. de Leeuw and S. J. van Tongeren, "The Quantum Deformed Mirror TBA I," JHEP 1210 (2012) 090 [arXiv:1208.3478 [hep-th]]; “The Quantum Deformed Mirror TBA II," JHEP [JHEP 1302 (2013) 012] [arXiv:1210.8185 [hep-th]].

[18] S. Schafer-Nameki, M. Yamazaki and K. Yoshida, "Coset Construction for Duals of Non-relativistic CFTs," JHEP 0905 (2009) 038 [arXiv:0903.4245 [hep-th]].

[19] I. V. Cherednik, "Relativistically Invariant Quasiclassical Limits Of Integrable TwoDimensional Quantum Models," Theor. Math. Phys. 47 (1981) 422 [Teor. Mat. Fiz. 47 (1981) 225].

[20] L. D. Faddeev and N. Y. Reshetikhin, "Integrability of the principal chiral field model in (1+1)-dimension," Annals Phys. 167 (1986) 227.

[21] J. Balog, P. Forgacs and L. Palla, "A Two-dimensional integrable axionic sigma model and T duality," Phys. Lett. B 484 (2000) 367 hep-th/0004180. 
[22] I. Kawaguchi and K. Yoshida, "Hidden Yangian symmetry in sigma model on squashed sphere," JHEP 1011 (2010) 032. arXiv:1008.0776 [hep-th]].

[23] D. Orlando, S. Reffert and L. I. Uruchurtu, "Classical integrability of the squashed three-sphere, warped $\mathrm{AdS}_{3}$ and Schrödinger spacetime via T-Duality," J. Phys. A 44 (2011) 115401. arXiv:1011.1771 [hep-th]].

[24] I. Kawaguchi, D. Orlando and K. Yoshida, "Yangian symmetry in deformed WZNW models on squashed spheres," Phys. Lett. B 701 (2011) 475. [arXiv:1104.0738 [hep-th]].

[25] I. Kawaguchi and K. Yoshida, "Hybrid classical integrability in squashed sigma models," Phys. Lett. B 705 (2011) 251 [arXiv:1107.3662 [hep-th]], "Hybrid classical integrable structure of squashed sigma models: A short summary," J. Phys. Conf. Ser. 343 (2012) 012055 [arXiv:1110.6748 [hep-th]].

[26] I. Kawaguchi, T. Matsumoto and K. Yoshida, "The classical origin of quantum affine algebra in squashed sigma models," JHEP 1204 (2012) 115 [arXiv:1201.3058 [hep-th]].

[27] I. Kawaguchi, T. Matsumoto and K. Yoshida, "On the classical equivalence of monodromy matrices in squashed sigma model," JHEP 1206 (2012) 082 arXiv:1203.3400 [hep-th]].

[28] I. Kawaguchi and K. Yoshida, "Classical integrability of Schrodinger sigma models and q-deformed Poincare symmetry," JHEP 1111 (2011) 094 [arXiv:1109.0872 [hep-th]], "Exotic symmetry and monodromy equivalence in Schrodinger sigma models," JHEP 1302 (2013) 024 [arXiv:1209.4147 [hep-th]];

[29] I. Kawaguchi, T. Matsumoto and K. Yoshida, "Schroedinger sigma models and Jordanian twists," JHEP 1308 (2013) 013 [arXiv:1305.6556 [hep-th]].

[30] D. Orlando and L. I. Uruchurtu, "Integrable Superstrings on the Squashed Threesphere," JHEP 1210 (2012) 007 [arXiv:1208.3680 [hep-th]].

[31] B. Basso and A. Rej, "On the integrability of two-dimensional models with $U(1) \times$ $S U(N)$ symmetry," Nucl. Phys. B 866 (2013) 337 [arXiv:1207.0413 [hep-th]].

[32] F. Delduc, M. Magro and B. Vicedo, "On classical q-deformations of integrable sigmamodels," arXiv:1308.3581 [hep-th]. 
[33] F. Delduc, M. Magro and B. Vicedo, "An integrable deformation of the $\mathrm{AdS}_{5} \times \mathrm{S}^{5} \mathrm{su}^{-}$ perstring action," arXiv:1309.5850 [hep-th].

[34] V. Ginzburg, M. Kapranov and E. Vasserot, "Langlands reciprocity for algebraic surfaces," Math. Res. Lett. 2 (1995) 147.

[35] J. M. Maillet, "New integrable canonical structures in two-dimensional models," Nucl. Phys. B 269 (1986) 54.

[36] V. G. Drinfel'd, "Hopf algebras and the quantum Yang-Baxter equation," Sov. Math. Dokl. 32 (1985) 254.

[37] V. G. Drinfel'd, “Quantum groups," J. Sov. Math. 41 (1988) 898 [Zap. Nauchn. Semin. 155, 18 (1986)].

[38] M. Jimbo, "A $q$ difference analog of $U(g)$ and the Yang-Baxter equation," Lett. Math. Phys. 10 (1985) 63.

[39] N. Reshetikhin, "Multiparameter quantum groups and twisted quasitriangular Hopf algebras," Lett. Math. Phys. 20 (1990) 331.

[40] M. Kruczenski, "Spin chains and string theory," Phys. Rev. Lett. 93 (2004) 161602 hep-th/0311203.

[41] W. -Y. Wen, "Spin chain from marginally deformed $\mathrm{AdS}_{3} \times \mathrm{S}^{3}$," Phys. Rev. D 75 (2007) 067901 hep-th/0610147.

[42] T. Kameyama and K. Yoshida, "String theories on warped AdS backgrounds and integrable deformations of spin chains," JHEP 1305 (2013) 146 [arXiv:1304.1286 [hep-th]].

[43] D. Israel, C. Kounnas, D. Orlando and P. M. Petropoulos, "Electric/magnetic deformations of $\mathrm{S}^{3}$ and $\mathrm{AdS}_{3}$, and geometric cosets," Fortsch. Phys. 53 (2005) 73 hep-th/0405213.

[44] D. Orlando and L. I. Uruchurtu, "Warped anti-de Sitter spaces from brane intersections in type II string theory," JHEP 1006 (2010) 049 [arXiv:1003.0712 [hep-th]].

[45] W. Song and A. Strominger, "Warped AdS3/Dipole-CFT Duality," JHEP 1205 (2012) 120 arXiv:1109.0544 [hep-th]]. 
[46] S. Detournay, J. M. Lapan and M. Romo, "SUSY Enhancements in (0,4) Deformations of $\mathrm{AdS}_{3} / \mathrm{CFT}_{2}, "$ JHEP 1201 (2012) 006 [arXiv:1109.4186 [hep-th]].

[47] S. Detournay and M. Guica, "Stringy Schroedinger truncations," JHEP 1308 (2013) 121 arXiv:1212.6792.

[48] P. Karndumri and E. O Colgain, "3D Supergravity from wrapped D3-branes," JHEP 1310 (2013) 094 [arXiv:1307.2086 [hep-th]]. 